\title{
The Evaluation of Multi-Objective Evolutionary Algorithms for a Maritime Domain Awareness Problem
}

\author{
by \\ Tolulope Oyinkan Akinbulire, B.Sc. \\ A thesis submitted to the \\ Faculty of Graduate and Postdoctoral Affairs \\ in partial fulfillment of the requirements for the degree of
}

Master of Applied Science in Electrical and Computer Engineering

Ottawa-Carleton Institute for Electrical and Computer Engineering

Department of Systems and Computer Engineering

Carleton University

Ottawa, Ontario

June, 2019

(C) Copyright

Tolulope Oyinkan Akinbulire, 2019 
The undersigned hereby recommends to the

Faculty of Graduate and Postdoctoral Affairs

acceptance of the thesis

\section{The Evaluation of Multi-Objective Evolutionary Algorithms for a Maritime Domain Awareness Problem}

submitted by Tolulope Oyinkan Akinbulire, B.Sc.

in partial fulfillment of the requirements for the degree of

Master of Applied Science in Electrical and Computer Engineering

Professor Howard Schwartz, Thesis Supervisor

Professor Yvan Labiche, Chair,

Department of Systems and Computer Engineering

Ottawa-Carleton Institute for Electrical and Computer Engineering

Department of Systems and Computer Engineering

Carleton University

June, 2019 


\section{Abstract}

Illegal, unreported and unregulated (IUU) fishing is a worldwide issue causing local and global financial losses, depleting natural resources and causing undue pressure upon the fishing industry. It is estimated that IUU fishing accounts for about $30 \%$ of all fishing activity worldwide, both on open oceans and within national exclusive economic zones. Responding to IUU fishing incidents is of paramount importance to law enforcement and marine environment protection organizations.

We employ an optimization approach to the IUU problem by applying Evolutionary Multi-Objective Optimization solution techniques (EMOO) to automatically generate a set of promising candidate responses once an IUU fishing event has been identified. Four of the most frequently cited EMOO algorithms were used to explore the trade-off among three conflicting decision objectives, namely: the proximity to the target (IUU fishing vessel), the total cost of the response for all engaged assets and the probability of confirming the detection of the offending vessel inside the fishing zone, which is important for prosecution purposes. The inherent differences between the algorithms are reviewed in detail to determine which of them was best suited in a complex application such as the IUU problem.

We illustrate the proposed methodologies with a simulated scenario along the Canadian Atlantic coast and discuss some of the automatically generated responses that are offered to the decision maker for their consideration. To the best of our 
knowledge, this is the first time any multi-objective optimization technique has been employed in generating responses to IUU fishing incidents and the building blocks of our proposed methodology here is generic enough that it can be easily extrapolated to other complex domain applications. 


\section{Acknowledgments}

I thank God for the grace to start and complete my master's program; for the knowledge, understanding and wisdom in my research work, for keeping me and all that are mine and for provision through it all.

I would like to thank parents for all their love and support financially, prayerfully, materially and otherwise and my sisters for always rooting for me and praying with me.

My sincere gratitude to my thesis supervisor, Prof. Howard Schwartz for his advice, encouragement, patience, thoroughness, guidance and funding throughout my graduate program.

I also want to express my appreciation to Rafael Falcon and Rami Abielmona and everyone at Larus Technologies for the opportunity to work with them throught the NSERC Engage program.

Finally, I'd like to thank my colleagues Esther Akinwumi and Walter Aburime for their friendship and fruitful interactions. Thanks also to the professors and staff of the department. 


\section{Table of Contents}

Abstract $\quad$ iii

Acknowledgments $\quad$ v

Table of Contents $\quad$ vi

List of Tables $\quad$ x

List of Figures $\quad$ xi

Nomenclature $\quad$ xiv

1 Introduction 1

1.1 Overview . . . . . . . . . . . . . . . . . . . 1

1.2 Motivation ........................... 2

1.3 Problem Statement . . . . . . . . . . . . . . . . . 3

1.4 Literature Review . . . . . . . . . . . . . . . . . . . . . . . 4

1.4.1 Responding to risks in the Maritime Domain . . . . . . . . . . 4

1.4.2 Multi-objective evolutionary algorithms . . . . . . . . . . 8

1.5 Contributions . . . . . . . . . . . . . . . . . . . 13

1.6 Thesis Organization . . . . . . . . . . . . . . . . . . 13 
2.1 Introduction . . . . . . . . . . . . . . . . . . 15

2.2 Elements of genetic algorithms . . . . . . . . . . . . . . 16

2.2.1 Population and Encoding . . . . . . . . . . . 16

2.2.2 Selection and Fitness Evaluation . . . . . . . . . . . . . 17

2.2.3 Recombination or Crossover . . . . . . . . . . . . . 18

2.2.4 Mutation .................... . . 20

2.2.5 Termination Criteria . . . . . . . . . . . . . . 20

2.3 A brief example ...................... . . 21

3 Evolutionary Multi-Objective Optimization 30

3.1 Introduction . . . . . . . . . . . . . . . . . 30

3.2 Multi-Objective Optimization . . . . . . . . . . . . . . . . 30

3.2.1 The Concept of Dominance . . . . . . . . . . . . . . 31

3.2.2 Pareto-Optimal Solution . . . . . . . . . . . . . . 33

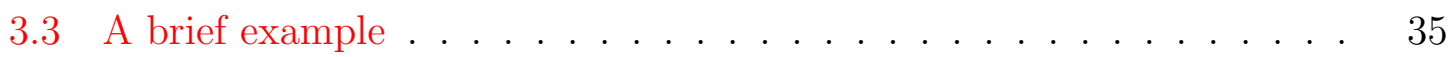

3.3.1 Elitist Non-dominated Sorting Genetic algorithm (NSGA-II) 37

3.3.1.1 Initialization .............. 38

3.3.1.2 Non-dominated sorting operation . . . . . . . . . 39

3.3.1.3 Crowding distance assignment operation . . . . . . 49

3.3.1.4 Create offspring population set . . . . . . . 54

3.3.1.5 Evolve next parent population . . . . . . . . . 55

3.3.2 Reference point-based non-dominated sorting algorithm $(\mathrm{NSGA}-\mathrm{III}) \ldots \ldots \ldots \ldots$. . . . . . . . . . . . . . 60

3.3.2.1 Non-dominated sorting . . . . . . . . . . . 60

3.3.2.2 Determination of reference points . . . . . . . . . 61 
3.3.2.3 Adaptive normalization of population members . . . 66

3.3.2.4 Association operation . . . . . . . . . . . . 72

3.3.2.5 Diversity-preservation operation . . . . . . . . . 77

3.3.3 The improved Strength Pareto Evolutionary Algorithm (SPEA2) 82

3.3.3.1 Initialize parent population and archive set . . . . 82

3.3.3.2 Compute fitness value attributes . . . . . . . 84

3.3.3.3 Environmental selection process . . . . . . . . . . 90

3.3.4 Region-based Pareto Envelope-based Selection Algorithm $(\mathrm{PESA}-\mathrm{II}) \ldots \ldots \ldots \ldots \ldots \ldots \ldots$

3.3.4.1 Initialization . . . . . . . . . . . . 94

3.3.4.2 Construct a hyper-grid . . . . . . . . . . . . . 96

3.3.4.3 Create new internal population . . . . . . . . . 100

3.4 Comparing the algorithms . . . . . . . . . . . . . . . 103

3.4.1 Performance of the algorithms . . . . . . . . . . . 103

3.4.2 Effects of the diversity operations of the algorithms . . . . 108

4 Simulation Analysis and Results 112

4.1 Introduction . . . . . . . . . . . . . . . . . . . . . . 112

4.2 Generating potential responses to IUU fishing events with MOEAs . . 112

4.2 .1 The IUU scenario . . . . . . . . . . . . . . . . . . . . . . 113

4.2.1.1 The Geohash system . . . . . . . . . . . . . 115

4.2.2 The multi-objective optimization problem . . . . . . . 118

4.2.2.1 Solution encoding. . . . . . . . . . . . . . 118

4.2.2.2 Decision objectives . . . . . . . . . . . . . 119

4.2.2.3 Algorithm parameters . . . . . . . . . . . . . 121

4.2 .3 Simulation results . . . . . . . . . . . . . . . . . . . 122 
4.2.3.1 The NSGA-II algorithm . . . . . . . . . . . . 122

4.2.3.2 The NSGA-III algorithm . . . . . . . . . . . . . 124

4.2.3.3 The SPEA2 algorithm . . . . . . . . . . . . 126

4.2.3.4 The PESA-II algorithm . . . . . . . . . . . . 127

4.2.4 Comparing the algorithms . . . . . . . . . . . . . 128

4.2.4.1 Performance of the algorithms using the Hypervolume metric . . . . . . . . . . . . . 128

4.2.4.2 Effects of the diversity operations of the algorithms . 130

5 Conclusion and Future work

5.1 Conclusion . . . . . . . . . . . . . . . . . . . . 135

5.2 Future work . . . . . . . . . . . . . . . . 136

List of References 137 


\section{List of Tables}

3.1 Dominated count and domination set parameters . . . . . . . . 41

3.2 Updated dominated count of the solutions in solution 2's domina-

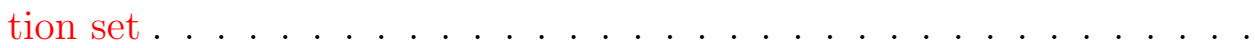

3.3 Updated dominated count of the solutions in solution 7's domination set . . . . . . . . . . . . . . . .

3.4 Updated dominated count and domination set parameters of the solutions in the first non-dominated set, $\mathcal{F}_{1} \ldots \ldots \ldots$

3.5 Updated dominated count and domination set parameters of the solution in the second non-dominated set, $\mathcal{F}_{2} \ldots \ldots$

3.6 Updated dominated count and domination set parameters of the solutions in the third non-dominated set, $\mathcal{F}_{3} \ldots \ldots \ldots$

3.7 Updated dominated count and domination set parameters of the solutions in the fourth non-dominated set, $\mathcal{F}_{4} \ldots \ldots . . \ldots 47$

3.8 Strength values for solutions in combined population in equation 3.60

3.9 Raw fitness values for solutions in combined population in equation 3.6087

3.10 Average diversity operation count . . . . . . . . . . . . . . 110

4.1 Asset Features . . . . . . . . . . . . . . . . . . . . . . . . . 114

4.2 Geohash precision cell dimensions . . . . . . . . . . . . . . . 118

4.3 Average diversity operation count . . . . . . . . . . . . . . 132 


\section{List of Figures}

2.1 Function curve of $f(x)=x^{2} \ldots \ldots \ldots \ldots \ldots \ldots$

2.2 Binary tournament selection operation to produce an offspring . . . . 23

2.3 The selection process . . . . . . . . . . . . . . . . . . . . 24

2.4 Genetic algorithm flow chart . . . . . . . . . . . . . . . . 29

3.1 A sample decision space to illustrate dominance . . . . . . . . . . 32

3.2 Graphical depiction of pareto-optimal set and pareto-optimal front . . 34

3.3 Graphical illustration of the objective functions in equation $3.2 \ldots \quad 36$

3.4 Closer view of the point at which the functions in equation 3.2 intercept 36

3.5 NSGA-II elitism approach $\ldots \ldots \ldots \ldots \ldots \ldots$

3.6 Plot of members of the parent population with the solutions close to the origin magnified $\ldots \ldots \ldots \ldots \ldots$

3.7 Pareto front for the first non-dominated front . . . . . . . . . 50

3.8 Pareto-optimal front showing solution to equation 3.2 using the NSGA-

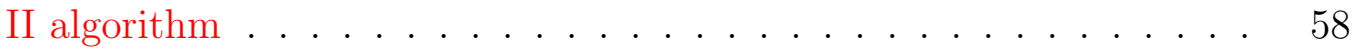

3.9 Tree diagram for generating reference points for a 3-objective problem with $\delta=0.25 \ldots \ldots \ldots \ldots \ldots \ldots \ldots \ldots \ldots \ldots$

3.10 Reference point coordinates on a normalized hyper plane for a 3objective problem with $\delta=0.25 \ldots \ldots \ldots \ldots \ldots$. . . . . 64

3.11 Possible values for $\beta_{1} \ldots \ldots \ldots \ldots \ldots \ldots \ldots$ 
3.12 Possible values for $\beta_{2} \ldots \ldots \ldots \ldots \ldots \ldots \ldots$

3.13 Plot of the reference points on the normalized space . . . . . . 66

3.14 Plot of the normalized space showing the normalized objective points 71

3.15 Plot of the normalized space showing the hyperplane and the reference

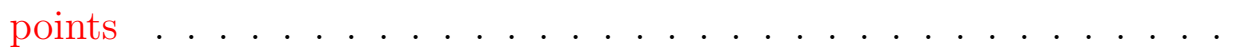

3.16 Illustration of how population members are associated with reference

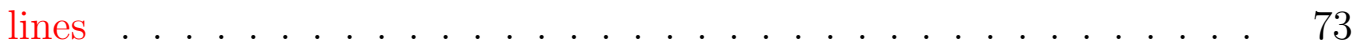

3.17 Plot of the reference lines $\ldots \ldots \ldots \ldots \ldots \ldots$

3.18 Plot showing normalized solutions associated to reference lines . . . . 77

3.19 Pareto-optimal front showing solution to equation 3.2 using NSGA-III

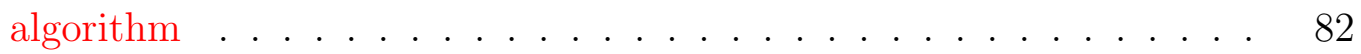

3.20 Pareto-optimal front showing solution to equation 3.2 using SPEA2

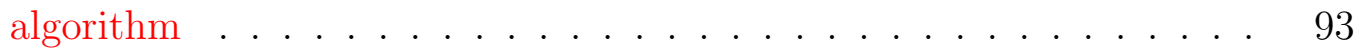

3.21 Illustration of the crowding hyperbox grid in a 2-objective problem . 96

3.22 Illustration of a $k$ th objective grid construction $\ldots \ldots \ldots$. . . 97

3.23 Graphical illustration of the hyper-grid for the external population in equation $3.75 \ldots \ldots \ldots \ldots \ldots \ldots$

3.24 Pareto-optimal front showing solution to equation 3.2 using PESA-II algorithm . . . . . . . . . . . . . . . . . 102

3.25 Graphical illustration of a hypervolume . . . . . . . . . . . . . . 104

3.26 Graphical plot of the hypervolume metric . . . . . . . . . . . 107

3.27 Hyper volume runtime dynamics for the NSGA-II algorithm . . . . 108

3.28 Hyper volume runtime dynamics for the NSGA-III algorithm . . . . . 109

3.29 Hyper volume runtime dynamics for the SPEA2 algorithm . . . . . . 109

3.30 Hyper volume runtime dynamics for the PESA-II algorithm . . . . . 109

3.31 Pareto front for the 4 algorithms using a small population size . . . . 111 
3.32 Another pareto front using a small population size . . . . . . . . . . . 111

4.1 Initial IUU Scenario . . . . . . . . . . . . . . . . . . . 113

4.2 Geohash precision example . . . . . . . . . . . . . . 115

4.3 Geohash precision example . . . . . . . . . . . . . 116

4.4 Geohash precision example . . . . . . . . . . . . . 116

4.5 Geohash precision example . . . . . . . . . . . . . . 117

4.6 A bi-layer encoded potential response member of the population ‘ 119

4.7 Geohash encoding of the fishing zone and the assets . . . . . . . . . 121

4.8 Pareto front generated using the NSGA-II algorithm . . . . . . . . . 123

4.9 Graphical illustration of a solution in the NSGA-II pareto front . . . 123

4.10 Pareto front generated using the NSGA-III algorithm . . . . . . . . . 124

4.11 Graphical illustration of a solution in the NSGA-III pareto front . . . 125

4.12 Pareto front generated using the SPEA2 algorithm . . . . . . . . . 125

4.13 Graphical illustration of a solution in the SPEA2 pareto front . . . . 126

4.14 Pareto front generated using the PESA-II algorithm . . . . . . . . . . 127

4.15 Graphical illustration of a solution in the PESA-II pareto front . . . . 128

4.16 Graphical plot of the hypervolume metric for the IUU scenario . . . . 131

4.17 Pareto front produced using a small population size . . . . . . . . . . 134 


\section{Nomenclature}

\section{List of Abbreviations}

\begin{tabular}{cc} 
Abbreviation & Meaning \\
\hline \hline MDA & Maritime Domain Awareness \\
IUU & Illegal, unreported and unregulated \\
SAR & Search and Rescue \\
CIP & Critical Infrastructure Protection \\
RMF & Risk Management Framework \\
CTRR & Common Contextual Risk Representation \\
CoA & Course of Action \\
AIS & Automatic Identification System \\
LFFD & Local Fishing, Foreign Delivery \\
EEZ & Exclusive Economic Zone \\
ML & Machine Learning \\
RL & Reinforcement Learning \\
& xiv
\end{tabular}


FACL

Fuzzy Actor-Critic Learning

MOOP Multi-Objective Optimization Problem

MCDP Multi-Criteria Decision Problem

EMOO Evolutionary Multi-Objective Optimization

MOEA Multi-Objective Evolutionary Algorithm

NFE Number of Function Evaluations

LFA Lobster Fishing Area

UAV Unmanned Aerial Vehicle

CPK Cost per Kilometer

DTT Distance to Target

PTT Proximity to Target

\section{List of Symbols}

\begin{tabular}{cc} 
Sybmol & Definitoin \\
\hline \hline$\sigma_{\text {share }}$ & Sharing Parameter \\
$M$ & Number of Objectives \\
$N$ & Population Size \\
$P_{0}$ & Initial Parent Population \\
$\prec$ & Dominance Operator
\end{tabular}




\begin{tabular}{|c|c|}
\hline$n_{p}$ & Dominated Count \\
\hline$S_{p}$ & Domination Set \\
\hline $\mathcal{F}_{i}$ & Non-dominated front $i$ \\
\hline$I_{D}$ & Crowding Distance Parameter \\
\hline$p$ & Number of Divisions \\
\hline$H$ & Number of Reference Points \\
\hline$\rho$ & Diversity Counting Operator \\
\hline$S(i)$ & Strength Value \\
\hline$R(i)$ & Raw Value \\
\hline$D(i)$ & Density Value \\
\hline$\sigma_{i}^{k}$ & Distance from solution $i$ to nearest $k$ th solution \\
\hline$F(i)$ & Total fitness value \\
\hline
\end{tabular}




\section{Chapter 1}

\section{Introduction}

\subsection{Overview}

The Maritime Domain can be described as all areas and things of, on, under, relating to, adjacent to, or bordering on a sea, ocean, or other navigable waterway, including all maritime related activities, infrastructure, people, cargo, and vessels and other

conveyances [1]. The sector acts as a core contributor to the socio-economic development of nations by supporting industries such as fishery, offshore oil and gas, passenger transportation, shipping, ports and marine tourism, amongst others.

Maritime Domain Awareness (MDA) can be defined as the situational knowledge of all activities and conditions that may directly or indirectly impact the safety, economy, and security of the maritime domain [2]. MDA processes involve combining domain knowledge acquired from various resources such as: technology (e.g. radars, satellite, etc.), people (e.g. marine vessel operators), history etc. Performing operations such as: sensing, capturing, storing, analyzing and processing on the domain knowledge to generate actionable intelligence that can be utilized in operations designed to respond to those activities and conditions that pose a risk to the maritime domain [2]. 
The International Maritime Organization's definition of risk follows the ISO 8402:1995/BS 4778 standard which defines risk as the combination of the probability, or frequency, of occurrence of a defined hazard and the magnitude of the consequences of the occurrence [3]. Some types of risk encountered in the maritime domain include: monitoring deployed sensors for threats (e.g. battery depletion, hardware tampering, software attacks etc), Search-and-Rescue (SAR) operations, piracy, Critical Infrastructure Protection (CIP) etc.

However, more importantly, is the process by which the risk is managed within maritime operations. A typical response operation usually involves the following processes: detection/identification of the risk posed to the domain, assessment of such risk, control/mitigation of the risk and monitoring the effectiveness of those controls [4].

\subsection{Motivation}

IUU fishing activities are largely responsible for dwindling marine livestock and the destruction of marine habitats worldwide. According to authors in [5] and [6], it is estimated that IUU fishing accounts for about $30 \%$ of all fishing activity worldwide, both in international open waters and within national EEZs. A report released by the Food and Agriculture Organization's Fisheries and Aquatic Department in 2016 estimates the financial losses caused by IUU fishing to be around $\$ 25$ billion per year [6]. At the national level, IUU operations impact negatively on the welfare of domestic fishing activities, cause Gross National Product loss to the economy, reduce future productivity and biodiversity of the marine ecosystem, increase pressure on endangered species etc. [6]. Responding to IUU fishing incidents is thus of the utmost importance to marine law enforcement and environment protection organizations in 
their pursuit of effective MDA [7].

Though generating responses to risks such as SAR, piracy, Vessels-in-Distress etc. have been well researched in the past, this, to the best of our knowledge, is the first time multi-objective evolutionary algorithm techniques have been applied in simulating responses to IUU fishing incidents in the maritime domain.

\subsection{Problem Statement}

Since the mid-eighties, several multi-objective evolutionary algorithms have been proposed capable of searching for multiple pareto-optimal solutions simultaneously in a single run. In spite of the many algorithms to choose from, it is difficult to determine the appropriate algorithm for a given problem because we lack extensive and quantitative comparative research studies of the algorithms. The few comparisons available to date (to the best of our knowledge) are mostly qualitative and restricted to (at most) two different algorithms; and quite often, the test problems are rela-

tively simple. As such, this thesis focuses on investigating the inherent differences in performance of some of these algorithms on a simple problem and also in a complex maritime domain awareness application.

Illegal, unreported and unregulated (IUU) fishing is one activity that is of major concern to MDA operations. The illegal term refers to fishing activities contrary to or forbidden by the laws and regulations of the governing fisheries authority; while the unreported term is descriptive of situations where the catch from a vessel's fishing activities have not been properly accounted to the relevant authorities; finally, the unregluated term implies fishing activities conducted in areas with little or no preservation laws and management measures to regulate catch [8]. 
Responding to an IUU activity can be quite challenging, mainly because the offending fishing vessel would have to be caught in the act so as to establish legal grounds for prosecution. To this end, this thesis focuses on generating adequate and realistic responses using computational intelligent approaches to two IUU fishing scenarios namely: Automatic Identification System (AIS) Gaps and Local Fishing, Foreign Delivery (LFFD).

The AIS gaps scenario typically describes a situation where a marine vessel maliciously turns off its AIS transponder - usually near sensitive or forbidden fishing zones - to avoid being detected while carrying out prohibited fishing activities. The LFFD scenario describes a situation where a marine vessel, though fishing in a country's Exclusive Economic Zone (EEZ) does not return to any port of the country for its catch to be audited.The AIS gaps scenario can be categorized under both illegal and unregulated fishing activities while the LFFD scenario falls under unregulated fishing activity.

\subsection{Literature Review}

This thesis proposes the use of evolutionary multi-objective algorithms in generating responses to the illegal, unreported and unregulated maritime domain awareness problem. As such, we will review literature on current computational techniques employed in generating responses to maritime domain awareness operations and key literature on multi-objective evolutionary algorithms.

\subsubsection{Responding to risks in the Maritime Domain}

Generating responses to commonly encountered risks in the maritime domain has been studied extensively in recent years. In that time, researchers have proposed different 
approaches to generate optimal responses appropriate in mitigating the threat $(\mathrm{s})$ on the domain.

In [9] an adaptive Risk Management Framework (RMF) was introduced to monitor threats to wireless sensor networks placed in unknown environments. The RMF uses a set of characterized risk features captured at multiple discrete intervals from continuous live data passed into it. The framework consists of four modules namely:

- A Risk Visualization module which displays the overall risk in the form of clusters corresponding to the sensor nodes in the risk feature space.

- A Risk Assessment module which "makes sense" of the input data by quantifying the perceived risk posed on the sensors. Here, the operator can also make changes to the perceived threat levels which when fed back to the system will update the situational picture of the risk feature space.

- A Risk Monitoring module which uses the output from the assessment module to trigger an alarm to the operator when a condition interpreted as harmful is detected.

- A Risk Forecasting module which updates the risk model based on the current snapshot of the risk space and any inputs from the operator.

- Finally, a Security Manager module that assigns actions to be taken based on the perceived risk.

Authors in [10] extended the idea presented in [9] to include a response selection mechanism that can be actuated on the environment to successfully deal with threats on one or more of the nodes. The proposed idea was used to simulate an effective and coordinated response to SAR operations in the shortest time possible while optimizing the available resources. This is achieved by using multi-criteria decision analysis in 
the response selection mechanism to select a subset of assets (out of the large number of types available) and their coordinated actions.

In place of wireless sensor networks, the authors in [11] proposed using Robotic Sensor Networks for their communication and actuation capabilities which can be highly effective when deployed in high-risk and dynamic environments (e.g. CIP). The autonomous robotic nodes are decentralized and can combine to mitigate any threats to the network. The first node to detect the threat becomes the auctioneer node and "advertises" it to the available robotic nodes in the network. The information on the "advert" contains the risk information detected to help each node make appropriate bids to participate in a risk-mitigation operation. Each robotic node is responsible for evaluating its bid metric (i.e. availability metric) for the risk mitigation task. Fuzzy logic was used in computing the bid metric which incorporated data such as: battery level, distance to the event and redundant coverage. The auctioneer node only considered the top bidder (those nodes with high bid metrics) and ends the bid when a minimum number of bids have been submitted. The risk is modelled as a multi-objective optimization problem and solved using the NSGA-II evolutionary algorithm to generate solutions consisting of the selected robotic nodes.

The authors in [12] proposed fusing data gotten from soft data sources (e.g. historical records, tweets etc.) with those gotten from hard data sources (e.g. sensor data) to get a more comprehensive perception of the risk posed to the domain. The combined data is then inputed to a modified version of the RMF introduced in [10]. The proposed modification is in form of an external module called the Common Contextual Risk Representation (CTRR) module. The CTRR performs natural language processing on the soft data to extract risk lexicon which will be used in building quantitative soft risk features which will be blended with those from the hard data sources and passed through the RMF. 
One downfall of the approach is that the reliability and timeliness of the soft data sources may reduce the input data intelligence when compared to using only hard data sources. To solve this problem, the authors in [13] proposed adding human intuition and perception through textual reports as another source of soft data. This methodology can be time consuming due to the human effort required to build the lexicon for the annotations.

To account for behavioural intents associated with Objects of Interest in the monitoring environment, the authors in [14] adapt the RMF introduced in [9] to include the ability to integrate a perceptual view of the risk sources. The intents of the objects of interest are deduced from high-level cognitive and behavioural knowledge in the form of anomalies. Risk features are then synthesized from anomalies and integrated into a more comprehensive RMF engine to draw out knowledge or evoke a response. When the anomaly has passed a given threshold, a Course of Action (CoA) generation process is initiated which will judge multiple assets which may have conflicting objectives. The evaluation is done using a multi-criteria decision algorithm to select amongst a myriad of possibilities a set of optimum set of responses. The responses can then be presented to the human operator who decides on the CoA to mitigate the intended risk. To improve the effectiveness of the proposed RMF, the authors in [15] explore how to augment the CoA using human-derived unstructured or semi-structured textual data which presented better optimum coordinated responses.

To improve the situational awareness of the environment, the authors in [16] expand the RMF to deal with contextual information and possess the ability to adequately map a risk to a well defined situational element e.g. piracy, bad weather etc. This is achieved by enhancing the RMF with the following modules: 
- A Contextual Knowledge Base module which contains information such as: regional weather reports, past incident reports, operational procedures and guidelines, organizational policies, history of successful responses to events, etc.

- A Dynamic Risk Assessment module which hosts a set of user-defined atomic risk models built using only relevant contextual information to accurately depict the current scenario.

- A Contextual Response Filter module which removes potential solutions from further consideration based on context-specific information such as: previous responses to similar scenarios, availability of resources, current conditions etc. This reduces the number of possible alternatives, thereby, reducing the computational complexity of the multi-criteria decision analysis module.

\subsubsection{Multi-objective evolutionary algorithms}

In many real-world optimization applications, we encounter a class of problems which are usually presented as multi-faceted optimization problems. These problems are usually defined with multiple objectives or criteria which are often competing and sometimes dependent on one another. Solving them does not produce a single optimal solution; but rather, a set of optimal alternative solutions known as pareto-optimal solutions. The pareto-optimal solutions are considered optimal in the sense that no other solutions are superior to any one of them when considering optimizing all objectives simultaneously. These class of problems are known as Multi-Objective Optimization problems (MOOP) or Multi-Criteria Decision problems (MCDP).

This class of optimization problems are presented in many real-life decision-making problems faced by people; a few examples include: purchasing a car, picking the right university to pursue a graduate degree program, picking the right insurance 
policy. These types of problems are also found in the fields of economics, finance, engineering, aviation among others. In the field of economics for example, the authors in [17] applied multi-objective optimization as an estimation tool in fisheries resource management and exploitation for the north sea fisheries zones. In engineering, the authors in [18] applied multi-objective optimization techniques in machining processes to determine optimal machining parameters such as cutting speeds, and feeding rates.

Over the years researchers have proposed different techniques to solving this class of optimization problems. In its earlier years, researchers settled for modifying and extending the classical methods of solving single-objective optimization problems, such as those presented in [19] and employed them in solving multi-objective optimization problems. These modified classical methods involve converting the multi-objective optimization problems into a composite single-objective optimization problem. This is done by scalarizing the set of objectives into a single-objective and multiplying each objective with a user-defined weight. The user-defined weight serves as a way of emphasizing one or more objectives based on the preference of the user. The classical methods are employed in solving the composite single-objective formalism multiple times while adjusting the user-defined weight with the hope of producing a different optimal solution at each simulation run.

Though presented as a simple idea, the classical approach has two major problems. One problem is in determining the values of the weights assigned to each objective in order to produce optimal results. That is, a user will have to try out many combinations of the weight values in order to achieve optimal results. Hence, a different set of weight values will produce a different optimal point each time and a uniform choice of weight values will not necessarily produce a uniform set of optimal solutions [20]. Another problem is that this procedure cannot be used to find pareto-optimal solutions which lie on sections of the pareto-optimal front which are defined by multiple 
local optimum points.

Unlike the classical methods, multi-objective evolutionary algorithms (MOEAs) simultaneously optimize the multiple objectives. These algorithms have the ability to find multiple pareto-optimal solutions in one single simulation run. The history of evolutionary algorithms can be traced back to the 1970s with every new algorithm boasting better performance over the previous ones. We will review - in subsequent paragraphs - some of the significant evolutionary algorithms proposed over the years.

In 1985, Schaffer et al. in [21] proposed the Vector Evaluated Genetic Algorithm (VEGA) to approximate multiple pareto-optimal non-dominated solutions in a single simulation run. The authors proposed splitting the population into $M$ randomly populated sub-populations (where $M$ is the number of objectives) in each iteration. Each sub-population member is then assigned fitness values based on one of the objective functions; hence each objective function will be responsible for evaluating the fitness values for members of at most one of the sub-population. The sub-populations are then merged together and the selection, crossover and mutation operators are applied to evolve the new generation.

Though the algorithm offers a relatively simple approach to solving multi-objective problems and generation multiple solutions in one simulation run, it mostly managed to find solutions at the extremes of the pareto front. On the other hand, the splitting and merging processes of the sub-populations is similar to evaluating the fitness average of each objective. Therefore, the expected fitness can be likened to a linear combination of the objectives (employed in the classical methods) where the assigned weights are dependent on the distribution of the population at each generation. Hence, the VEGA algorithm has relatively the same problems as the classical methods.

Over the years, variantions to the VEGA algorithm operations (such as those 
suggested in [22] and [23]) were proposed. A notable one introduced in 1997 by Lis and Eiben in [24] is called the Multi-Sexual Genetic Algorithm (MSGA). The algorithm proposes assigning to each individual of the population a gender where we have as many genders as the objectives in the problem. Each member of the population's fitness value is evaluated based on the objective relating to its gender.

In 1993, Fonseca and Fleming in [25] introduced the Multi-Objective Genetic Algorithm (MOGA) which proposes an extension of the pareto-based fitness assignment operation suggested by Goldberg et al. in [26], which gives the non-dominated individuals in the population an equal probability of reproduction. The MOGA presented a rank-based fitness assignment operation for the population members of each generation where the non-inferior or non-dominated members of the population are assigned rank 1 as the best solutions in the current population and the solutions are considered worse as the rank number increases. The algorithm also employed the conventional sharing parameter, $\sigma_{\text {share }}$, in ensuring diversity in the population.

In 1994, Srinivas and Deb in [27] proposed the Nondominated Sorting Genetic Algorithm (NSGA). The authors presented a non-dominated sorting procedure for ranking the members of the population but still maintainted the user-specified niche sharing parameter, $\sigma_{\text {share }}$, in maintaining diversity in the population. In 2002, Deb et al. in [28] proposed an improved version of the NSGA called the NSGA-II. NSGAII presented a more efficient way of sorting the population with a computational complexity of $O\left(M N^{2}\right)$ (where $M$ is the number of objectives and $N$ is the population size) which is better than NSGA's $O\left(M N^{3}\right)$ computational complexity.

The NSGA-II algorithm also replaced the user-defined sharing function approach to maintaining diversity in the population with a crowded comparison operation which adaptively sought to spread out the solutions in the population. In 2014, Deb et al. in [29] proposed a variation to the NSGA-II algorithm called the NSGA-III algorithm. 
The NSGA-III algorithm suggested using a reference-point-based approach to emphasizing the non-dominated solutions as well as maintain diversity in the population.

In 1998, Zitzler et al. in [30] presented the Strength Pareto Evolutionary Algorithm (SPEA) which employs an external archive set to store the non-dominant pareto-optimal solutions found in each generation. The selection, crossover and mutation operations are performed only on the external archive set. The algorithm employs fitness sharing in maintaining diversity among the population members and truncate the archive set if it exceeds its defined size. In 2001, Zitzler et al. in [31] proposed an improvement to the SPEA algorithm called the SPEA2 algorithm. The SPEA2 algorithm presented an improved fitness assignment operation which evaluated 3 fitness parameters. The algorithm also incorporated a nearest neighbour density estimation technique to maintaining diversity in the population. Both the fitness assignment and the nearest neighbour density estimation operations were used in the selection and truncation operations.

In 2000, Corne et al. in [32] introduced the Pareto Envelope-based Selection Algorithm (PESA) which also uses an external archive set to store the optimal solutions at each generation. In the PESA algorithm, the authors presented an idea where the objective space is divided into a hypergrid and fitness values are assigned to the members based on the number of individuals hyperboxes they occupy. Hence, solutions in the same hyper box will have the same fitness. Selection and diversity preservation operations are also based on the hypergrid where solutions in a less crowded hyperbox are preferred. In 2001, Corne et al. proposed the PESA-II algorithm in [33] as an extension to the PESA algorithm. The PESA-II algorithm presented a region-based selection technique where the occupied hyperboxes are assigned fitness values instead of the individual solutions in the population. During the selection operation, a less dense hyperbox is preferred (to ensure diversity) and a solution is randomly chosen 
from it for recombination and mutation operations.

\subsection{Contributions}

This thesis investigates generating adequate responses to incidents in a simulated IUU fishing scenarios. The main contributions of this thesis are:

- A detailed investigation of the inherent performance differences between four of the most cited Multi-Objective Evolutionary algorithms that have been proposed in the literature.

- A Multi-Objective Optimization (MOO) approach, where an IUU fishing incident was modelled as a multi-objective numerical optimization problem. and solved using several Evolutionary Multi-Objective Optimization (EMOO) algorithms to automatically generate a set of promising candidate responses once an IUU fishing event has been properly identified. The EMOO algorithms explore trade-offs among three conflicting decision objectives, namely: the proximity to the target (IUU fishing vessel), the total cost of the response for all engaged assets and the probability of confirming the detection of the offending vessel inside the unauthorized fishing zone, which is important for prosecution purposes [34].

In analyzing the approach, the hypothetical IUU scenarios and potential responses were illustrated using simulated scenarios along the Canadian-Atlantic coast.

\subsection{Thesis Organization}

The remainder of this thesis is structured as follows: 
- Chapter 2 presents background information single-objective optimization problems. It also introduces the concept of genetic algorithms and its operations for solving single-objective optimization problems.

- Chapter 3 introduces a class of optimization problems called the multi-objective optimization problems (MOOP) and discusses the four multi-objective evolutionary algorithms that are in this thesis.

- Chapter 4 presents and discusses the generated responses produced by applying the four multi-objective evolutionary algorithms to a simulated IUU fishing incident.

- Finally chapter 5 concludes the thesis with a summary of the contributions and projection ahead into the future work in the research area. 


\section{Chapter 2}

\section{Background on Genetic Algorithms}

\section{$2.1 \quad$ Introduction}

Genetic algorithms were invented by John Holland in the 1960s. He introduced them to study how an organism's generations adapt in nature to their environment; and also developed ways to apply them in computing systems as presented in his book: Adaptation in natural and artificial systems [35]. According to Holland, on a cellular scale, the genetic algorithm procedure involves transitioning from one population of chromosomes to a new one by incorporating operations inspired by genetics, namely: selection, recombination (or crossover) and mutation.

The selection operation is based on the principle of natural selection (i.e. only the fittest survives) where the strongest candidate chromosomes in the population will be chosen to mate in order to produce an offspring. The recombination or crossover operation follows the biological mating process where attributes from two chosen chromosomes are combined to create a new offspring chromosome. Finally, the mutation operation imitates the rare biological instances where the offspring chromosome contains attributes that are not present in any of the parent chromosomes. Basically, genetic algorithm procedures can be seen as a rough abstraction of the principles of 
biological evolution.

In the field of engineering, a genetic algorithm process can be described as a stochastic computational approach for solving numerical optimization problems by imitating the principles of evolution as it occurs in nature. For engineering applications, the genetic operations, namely: selection, recombination or crossover and mutation operators are modelled as mathematical abstractions of their respective biological concepts. The next section discusses the elements of genetic algorithms as related to engineering applications including the genetic operators and how their abstractions are generally modelled.

\subsection{Elements of genetic algorithms}

Over the years, many different types of genetic algorithms have been developed for solving numerical problems in engineering applications. Though the developed algorithms may vary in their approach to solving the problems, all of them have in common the following elements: a population, a selection operation based on the fitness of the population members, a recombination or crossover operation to produce a new offspring and a random mutation operation on a solution. In the following subsections we will discuss each element and describe its operation procedure. Please note there might be modifications to how these elements operate in other genetic algorithms, but the way they are explained here are based on how they are applied in this thesis.

\subsubsection{Population and Encoding}

A population can be described as a set of desired potential solutions that can be evolved by the algorithm to optimize the given numerical problem. Together, this set 
of solutions constitute a space of feasible solutions among which the desired solution resides (called the search space) for the genetic algorithm to navigate through to find a solution to the optimization problem. These potential solution(s) are feasible values that can substitute for the unknown/control decision variable parameter(s) in solving the optimization problem. For example, the decision variable value of the example in equation 2.3 is the variable $x$.

The way the solution members of the population set are represented is called encoding. There are a large number of encoding types but some commonly used ones are: binary encoding using $1 \mathrm{~s}$ and 0 s as shown in equation 2.1a, integer encoding using integer numbers $(\mathbb{Z})$ as shown in equation $2.1 \mathrm{~b}$, real encoding using real numbers $(\mathbb{R})$ as shown in 2.1c, bi-layered encoding as shown in equation $2.1 \mathrm{~d}$ used in complex optimization problems etc. The type of encoding chosen depends on the problem and the type of values the decision variable can take.

$$
\begin{aligned}
& A=\{0010110101011100011000110\} \\
& B=\left\{\begin{array}{lllll}
4 & 1 & -8 & -4 & -2
\end{array}\right\} \\
& C=\{3.14724 .0579-3.73011 .3236-4.0246\} \\
& D=\left\{\begin{array}{ccccc}
00101 & 10101 & 01110 & 00110 & 00110 \\
4 & 1 & -8 & -4 & -2
\end{array}\right\}
\end{aligned}
$$

\subsubsection{Selection and Fitness Evaluation}

This operation imitates biological natural selection principles of choosing two parent solution members to produce an offspring. The method for choosing the parents is known as the selection operator. There are many selector operators, but one common method is the binary tournament selection with replacement, where two parents are 
randomly selected from the population and the fitter one qualifies to take part in the recombination operation.

This binary tournament process involves assigning fitness values to the solutions in the population, randomly choosing two members from the population set and selecting the fitter of the candidate solutions based on their fitness values. There is no single method for assigning fitness values to the solutions and it all depends on the user, but one important thing to take note of is to assign the fitness values in such a way that it guides the evolution process in optimizing the objective of the problem (either minimization or maximization).

For numerical problems in this thesis, the fitness values of each solution member in the population is simply the evaluated objective function value obtained by substituting for the chosen decision variable(s) in the objective function. Thus, determining which of the selected parent members is fitter depends on the objective of the optimization problem. For example, if the objective is to minimize the objective function, the parent with the lesser objective function value is deemed fitter. Likewise, if the objective of the optimization problem is to maximize it, the parent solution with the larger value is said to be fitter. The binary tournament selection is repeated once again to obtained the second parent to be combined with the first to produce an offspring.

\subsubsection{Recombination or Crossover}

The recombination or crossover operation imitates the biological mating process in organisms where the produced offspring contains traits or characteristics from one or both parents. The method for combining the chosen parent solutions obtained in the selection operation is known as the recombination or crossover operation. Biologically, 
the mating process can either be be successful or not. The genetic algorithm formalizes the uncertainty of the mating process by defining a recombination/crossover rate parameter in the range $[0,1]$. The recombination rate can be described as the probability of applying the recombination/crossover operator to the selected parents to produce an offspring. For example, a rate of 0.7 means the crossover operation has a $70 \%$ chance of being applied, while a rate of 1.0 ensures that the crossover operation will always be applied. In situations where the crossover operation is not applied, the fittest individual in the parent population will be added to the next (offspring) population. At other occurrences, the next fittest individual will be added to the offspring population.

The type of crossover operator used depends largely on the type of encoding used to represent the desirable solution members of the population. There are many available recombination operators for each encoding type, but the choice of which one to use is based on the knowledgeable preference of the user. For the sake of this thesis we will consider only the recombination operators used for real-value encoding. Though there are many recombination operators, a simple one used for real-value encoding is the intermediate recombination function operator defined as shown in equation 2.2.

$$
\text { offspring }=\alpha \text { parent } 1+(1-\alpha) \text { parent } 2
$$

In equation 2.2, the variables parent1 and parent2 represent the solutions that were selected from the current parent population (e.g. the population set in equation 2.1c) during the selection operation procedure. The parameter $\alpha$ in equation 2.2 is the scaling factor chosen in the interval $[0,1]$. 


\subsubsection{Mutation}

Just like the biological process, sometimes on rare occasions we see qualities in the offspring that are not present in any of the parents. The mutation operation seeks to copy that feature by randomly changing the value of the offspring produced in the recombination operation. Just like in the recombination process, the user has to set the probability at which this operation will occur to model its uncertainty. The probability is set very low to replicate the rareness of the operation, usually between 0.01 and 0.1 . This operation can be useful in helping the algorithm avoid getting stuck in numerical problems that have multiple local optima. Mutation operation procedures also vary and are highly subjective to the problem and user preferences. For example in a real-valued encoding problem, the mutated offspring can be a random number generated within the constraint of the decision variable.

\subsubsection{Termination Criteria}

Finally, the user sets a termination criteria at which the algorithm will stop. This also depends on the user, two common criteria is for the algorithm to stop are: the maximum Number of Function Evaluations (NFEs) and the maximum number of generations (or iterations of the algorithm). The algorithm will continue another iteration of the algorithm process if the termination criteria is not met. 


\subsection{A brief example}

To highlight the methods associated with genetic algorithms, we present a simple example as follows:

\section{Objective:}

$$
\text { Minimize } f(x)=x^{2}
$$

\section{Constraint:}

$$
-10 \leq x \leq 10 \text { and } x \in \mathbb{R}
$$

Equation 2.3 shows an example of a numerical single-objective optimization problem that we will be solving using genetic algorithm. The objective of the problem is to minimize the function, hence, upon termination of the algorithm, we expect the final population set to contain real valued solution members that are very close to zero shown by the function curve figure 2.1. For the rest of this section, we will present how the elements of the genetic algorithm are employed in solving numerical problems such as these.

The first thing to do when using a genetic algorithm to solve an optimization problem is to generate an initial population set of potential solutions, $P_{0}$, called the initial parent population. This is usually done by populating the initial parent set with random encoded values (depending on the type of encoding chosen by the user) that can be taken by the decision variable(s). In the example problem in equation 2.3 , the variable parameter, $x$, is the problem's decision variable and is defined to take only real-value numbers, hence, we choose to use real-value encoding to represent the solution members of the population set. Equation 2.4 shows the randomly initialized parent population set $P_{0}$ of size $N=10$ generated from uniformly distributed numbers 


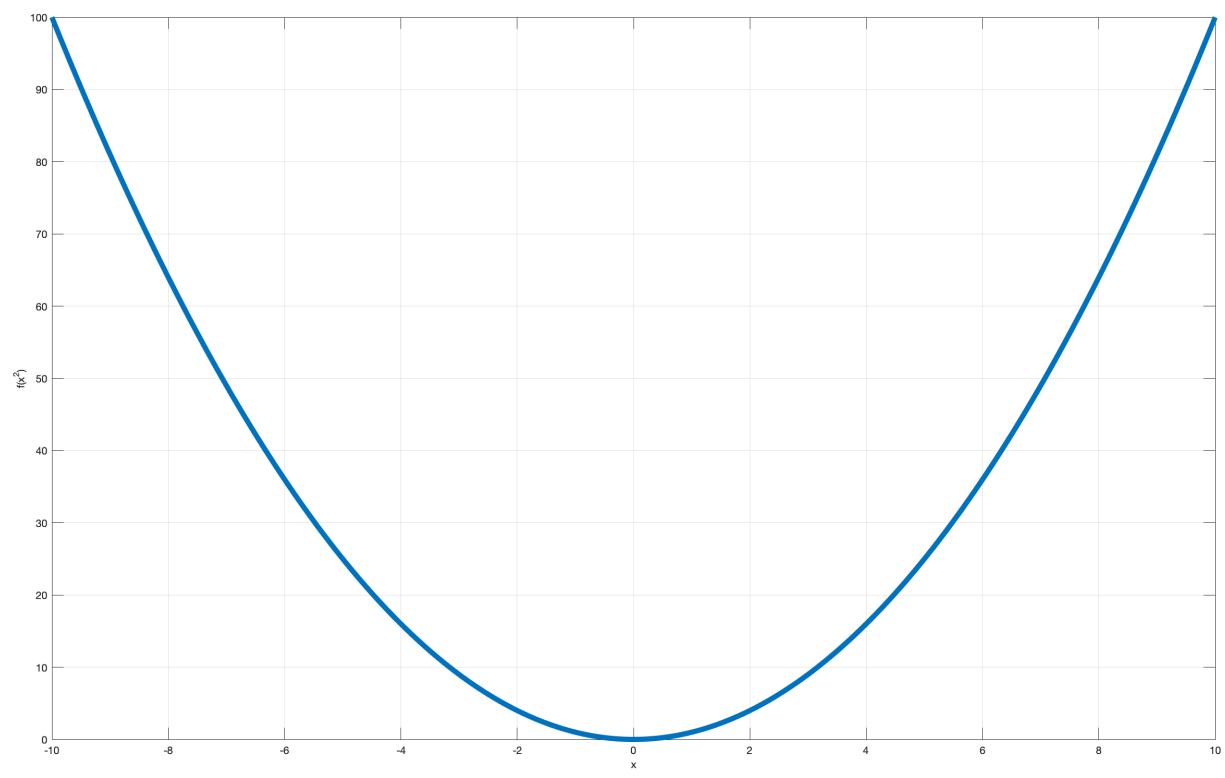

Figure 2.1: Function curve of $f(x)=x^{2}$

in the problem's defined constraint range $[-10,10]$.

$P_{0}=\{6.047,-6.814,-9.392,-9.817,-6.698,8.256,-1.545,-5.532,7.495,-3.862\}$

The next step in the algorithm is the fitness evaluation operation which is simply assigns fitness values to the members in the population. The fitness value for each member of the population is given by the function in equation 2.3 (i.e. $f(x)=x^{2}$ ). Hence, the fitness values assigned to each member of the initialized parent population set in equation 2.4 is given as:

$F_{0}=\{36.566,46.426,88.218,96.381,44.867,68.167,2.386,30.607,56.176,14.914\}$

To create the first offspring generation from the initialized parent population, we 
perform a binary tournament selection operation on the parent population set. Creating each offspring member requires two parents to recombine. The "binary" term in the selection operation describes the number of potential members from the parent population (i.e. 2 members) that will be considered in the selection operation for each parent of the offspring as shown in figure 2.2. The potential members participate in a fitness tournament to determine which of them will be chosen as a parent of the offspring as shown in 2.2 .

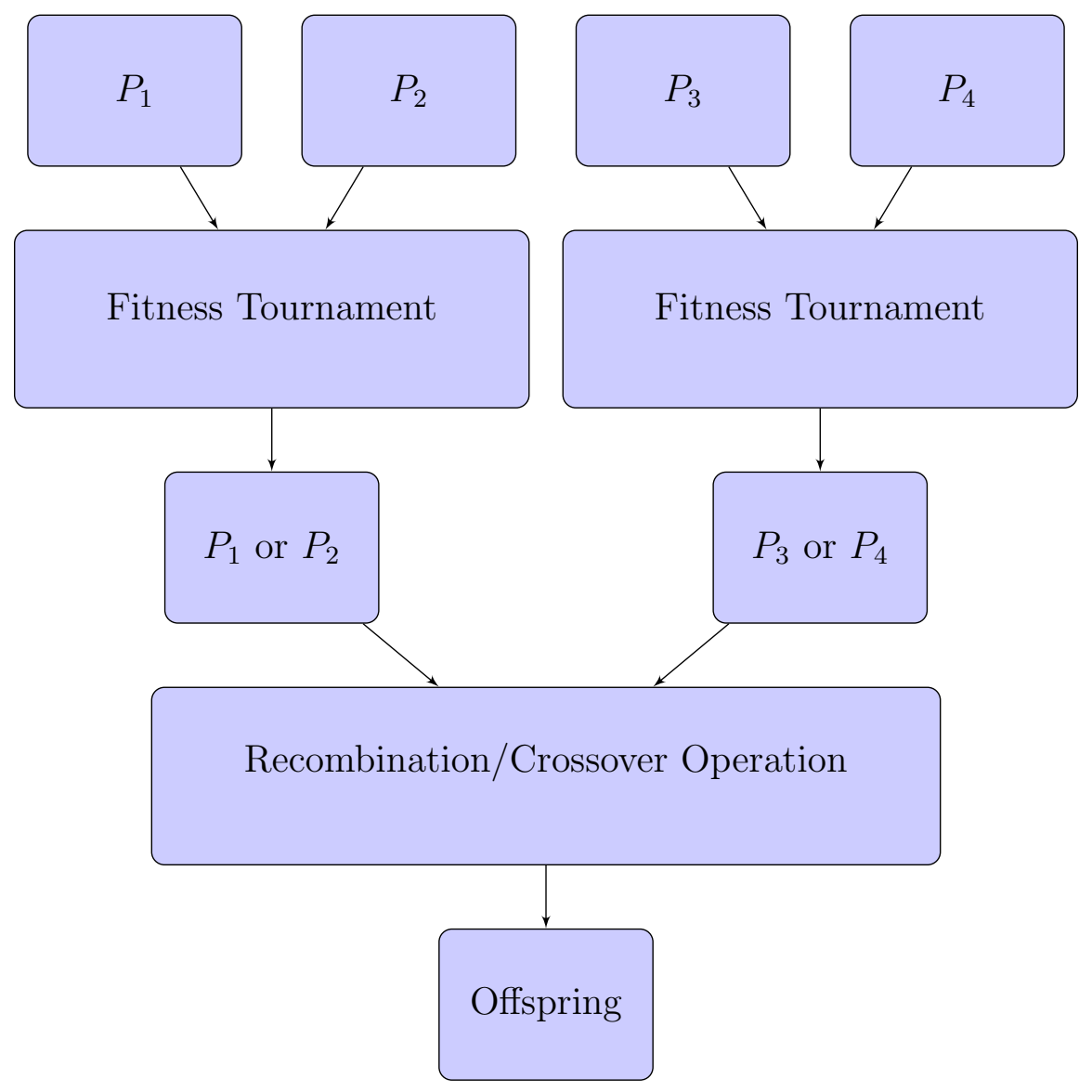

Figure 2.2: Binary tournament selection operation to produce an offspring

Thus, for the first offspring member in equation 2.8, the algorithm - based on a uniform distribution across the 10 members in the set - randomly selects member 3 
twice (i.e. $P_{0}^{3}=-9.392$ and $P_{0}^{3}=-9.392$ ) from the initial parent set in equation 2.4 to compete in the fitness tournament to determine which of them will be the first parent; likewise, the algorithm selects members 7 and 4 (i.e. $P_{0}^{7}=-1.545$ and $P_{0}^{4}=-9.817$ from equation 2.4) to determine which will be the other parent. The fitness tournament involves comparing the fitness values of the potential parent members based on the objective of the problem; hence in a minimization problem, the member with a smaller fitness value is chosen as the parent while in a maximization problem, the member with a larger fitness value is selected.
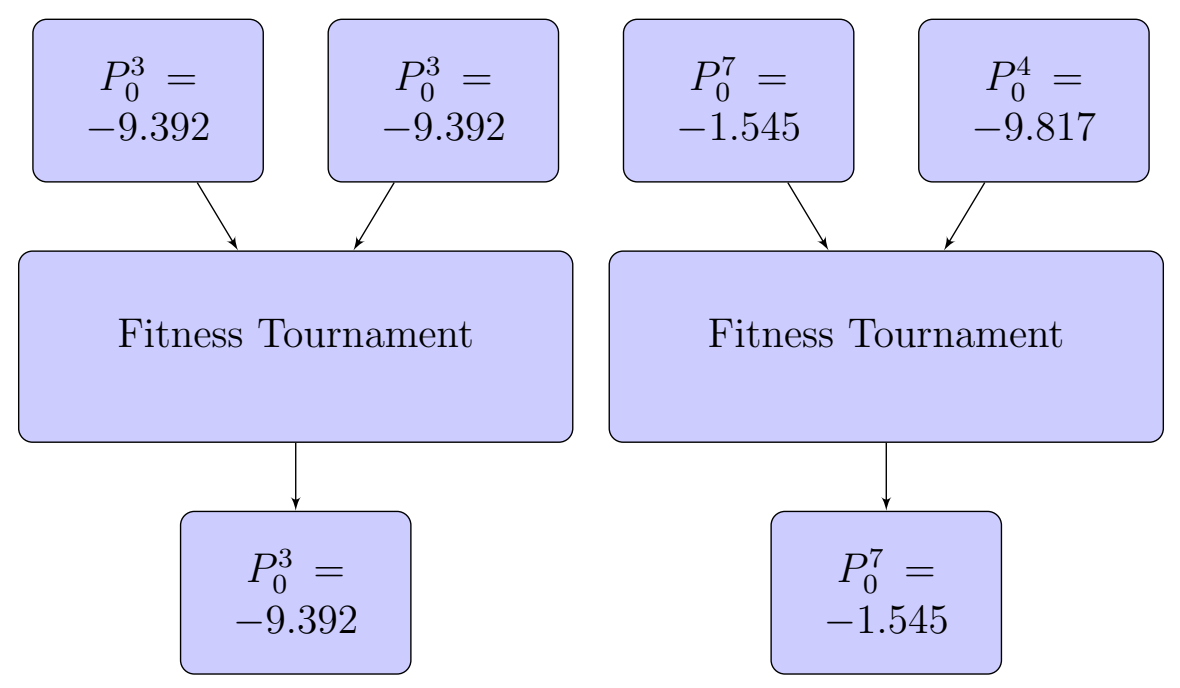

Figure 2.3: The selection process

Therefore, the algorithm compares the fitness values of the randomly chosen parent members in equation 2.5 based on the objective of the problem (i.e. minimization) and produces the member with a lower fitness value as a parent. Since the first pair of randomly chosen parents (i.e. $P_{0}^{3}=-9.392$ and $P_{0}^{3}=-9.392$ ) have the same fitness values (i.e. $F_{0}^{3}=88.218$ ), hence, the algorithm selects one of them at random and produces $P_{0}^{3}=-9.392$ as the first parent as shown in figure 2.3. Likewise, the fitness values of members 7 and 4 (i.e. $F_{0}^{7}=2.386$ and $F_{0}^{4}=96.381$ from equation 
2.5) are compared based on the objective of the problem producing member 7 (i.e. $\left.P_{0}^{7}=-1.545\right)$ with the lower fitness value of $F_{0}^{7}=2.386$ as the second parent as shown in figure 2.3.

Both selected parents (i.e. $P_{0}^{3}=-9.392$ and $P_{0}^{7}=-1.545$ ) chosen using the binary tournament selection operation will undergo a recombination operation as shown in figure 2.2 to produce an offspring solution. To imitate the biological uncertainty of producing an offspring from mating, we define a recombination/crossover rate parameter and set it to 0.7 ; which indicates a high probability for the recombination process to occur.

To formalize this, our algorithm generates a random uniform number in the range $[0.0,1.0]$; if the generated number is less than or equal to the crossover rate (i.e. 0.7$)$, both parents can recombine, otherwise both parents are discarded and the fittest individual in the parent population is added as a "survivor" to the new offspring population. At other occurrences where the generated number is greater than the crossover rate, the next fittest individual in the parent population is added to the offspring population. For the recombination operation, our algorithm uses the intermediate recombination function operator in equation 2.2 to produce a new offspring solution. Thus, to produce the first offspring solution in equation 2.8, the parents (i.e. $P_{0}^{3}=-9.392$ and $P_{0}^{7}=-1.545$ ) recombine as shown in equation 2.6 where the scaling factor $\alpha=0.5$.

$$
\begin{array}{r}
\text { offspring }=\alpha \text { parent } 1+(1-\alpha) \text { parent } 2 \\
0.5 \times-9.392+(1-0.5) \times-1.545 \\
\text { offspring }=-5.469
\end{array}
$$

Before adding the created offspring to the new population set, the algorithm 
applies a mutation operator to it. The mutation operator imitates the biological process where an offspring possesses traits or characteristics absent in both parents. In the case of our example problem, a mutation rate of 0.01 will be acceptable because the function has one local optimum (which is also the global optimum). To show the details of the mutation operation in our example we defined the mutation rate for the algorithm as 0.1 (to give an opportunity to present an instance of its occurrence).

The algorithm generates a uniform random number in the range $[0.0,1.0]$. If the number is less than or equal to the mutation rate, a new value is randomly generated for the offspring member in the uniform range $[-10,10]$ (i.e. the constraint defined for the decision variable, $x$ in equation 2.3). In our example, the mutation operator was applied by the algorithm in producing the $5^{\text {th }}$ member of the offspring population as shown in equation 2.7 where the two parents (parent $1=-1.545$ and parent $2=7.495$ ) initially combined to produce the offspring, 2.975 ; on applying the mutation operator, the offspring was changed to -8.054 .

$$
\begin{array}{r}
\text { parent1 }=-1.545 \\
\text { parent2 }=7.495 \\
\text { offspring }=\alpha \text { parent1 }+(1-\alpha) \text { parent2 } \\
0.5 \times-1.545+(1-0.5) \times 7.495 \\
\text { offspring }=2.975 \\
\text { On applying the mutation operator } \\
\text { offspring }=-8.054
\end{array}
$$

The genetic operators (i.e. selection, recombination and mutation operations) are repeatedly applied to the initial parent population until the new offspring population set of size $N=10$ is filled as shown in equation 2.8. The new offspring population is 
then set as the next generation's parent population, $P_{1}$, as shown in equation 2.8 .

$P_{1}=\{-5.469,-3.538,1.093,-1.545,-8.054,-6.756,2.386,-2.703,-5.338,4.914\}$

The genetic operators are again applied to the the new parent population to create another offspring population and the process is repeated until the termination criteria is met. For our example, the algorithm is set to terminate after 6 generations have been created. The next five generations evolved by the algorithm are as shown in equations $(2.9$ - 2.13) where we can see that the solution members of the offspring get closer to zero as the generation count increases.

$$
\begin{gathered}
P_{2}=\{0.421,1.403,0.177,7.352,-6.696,2.324,-2.58,-1.141,0.79,1.372\} \\
P_{3}=\{0.775,0.476,0.031,1.557,0.226,0.226,0.633,0.226,0.5,0.226\} \\
P_{4}=\{0.129,0.001,0.001,0.001,0.114,0.065,0,0.114,0,0\} \\
P_{5}=\{0.001,0.001,0,0,9.824,0,0,0,0,0\} \\
P_{6}=\{0,0,0,0,0,0,0,0,0,0\}
\end{gathered}
$$

Algorithm 1 summarizes the steps in solving the optimization problem in equation 2.3 using a simple genetic algorithm. The figure 2.4 shows a graphical representation of the genetic operation process in a genetic algorithm. 


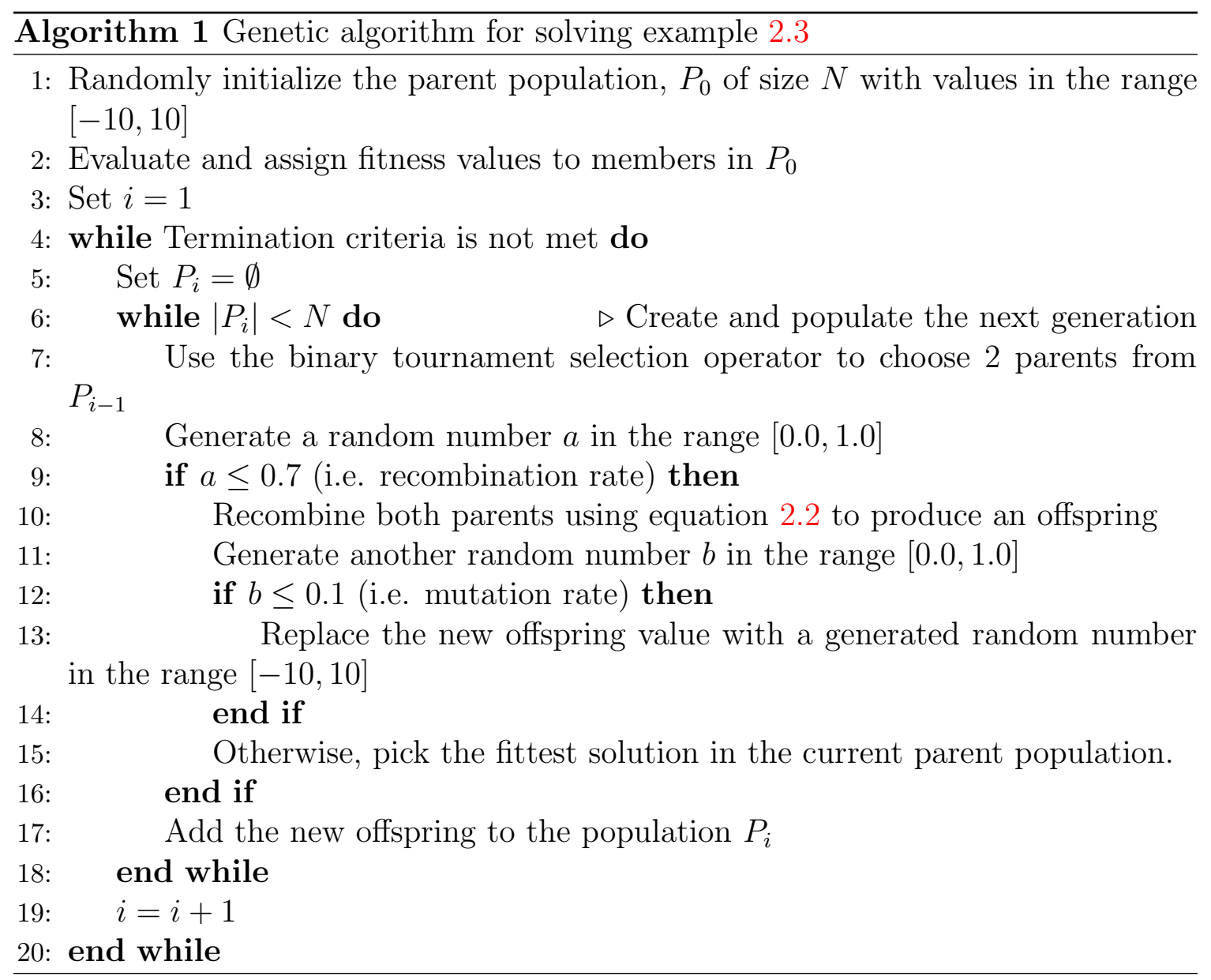




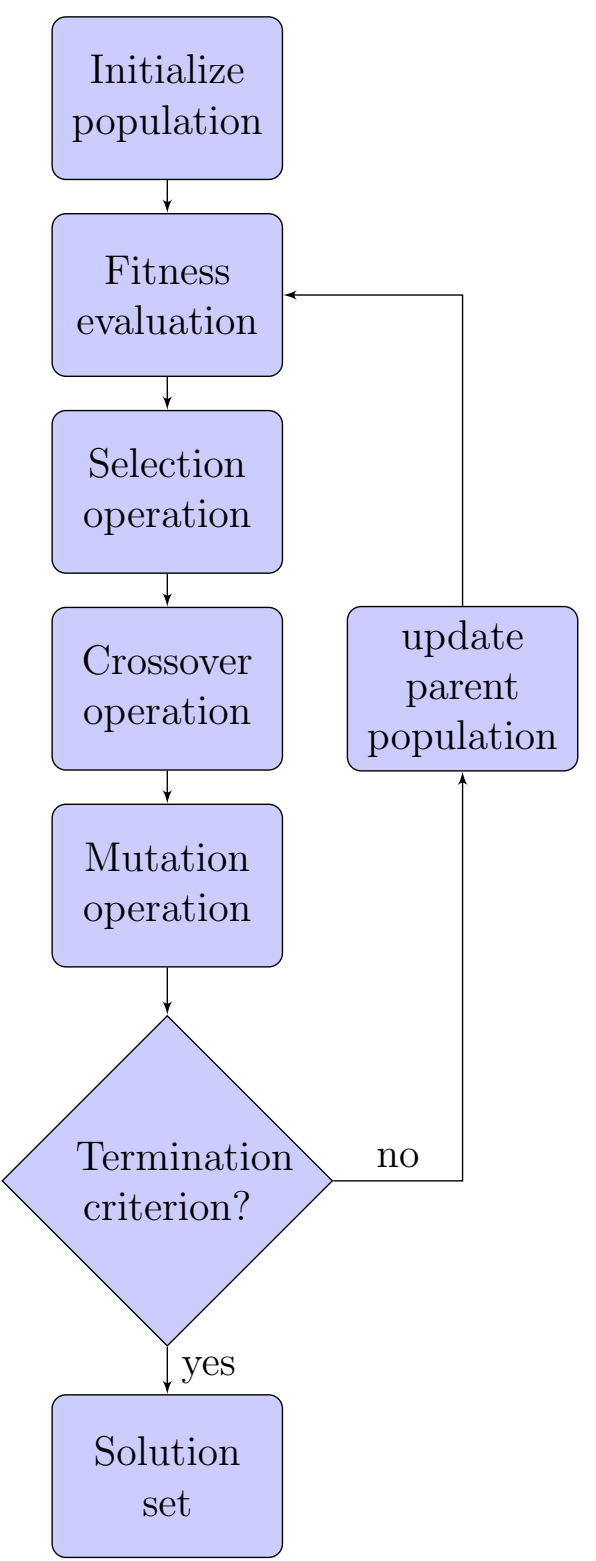

Figure 2.4: Genetic algorithm flow chart 


\section{Chapter 3}

\section{Evolutionary Multi-Objective Optimization}

\subsection{Introduction}

This chapter introduces a class of optimization problems called multi-objective optimization problems (MOOP) which adds another layer of complexity to the singleobjective optimization technique introduced in chapter 2 and presents in detail a select number of algorithms to solve them.

\subsection{Multi-Objective Optimization}

Multi-Objective Optimization problems (MOOP) otherwise known as multi-criteria decision making problems define a class of problems that involve finding optimal solutions to decision-making problems. These problems are mathematically modelled as optimization problems with more than one conflicting objective or criteria; where improving one objective leads to deterioration of another. 


\subsubsection{The Concept of Dominance}

Solving a multi-objective optimization problem involves applying genetic operators (i.e. the selection, crossover or recombination and mutation operators) to a set of potential solutions also known as a population. This in turn produces another set of more desirable solutions often called the offspring population.

$$
P_{x}=\left\{\left(f_{1}^{1}, f_{2}^{1} \ldots f_{m}^{1}\right),\left(f_{1}^{2}, f_{2}^{2} \ldots f_{m}^{2}\right), \ldots,\left(f_{1}^{n}, f_{2}^{n} \ldots f_{m}^{n}\right)\right\}
$$

Equation 3.1 shows a representation of a population of solutions where $P_{x}$ represents the population of solutions at generation $x ;\left(f_{1}^{n}, f_{2}^{n} \ldots f_{m}^{n}\right)$ represents a solution to the multi-objective problem, where $f_{1}^{n}, f_{2}^{n} \ldots f_{m}^{n}$ constitute the $n^{\text {th }}$ solution in the population set for objectives $1,2, \ldots, m$ respectively.

The desirability of a solution over another is determined by the concept of dominance. In multi-objective optimization algorithms, a solution $A$ is said to dominate another solution $B$ (i.e. $A \prec B$ ) if these two conditions hold true:

- The solution $A$ is no worse than solution $B$ in all objective function evaluations.

- The solution $A$ is strictly better than solution $B$ in at least one objective.

If any of these conditions is violated, it can be stated that solution $A$ does not dominate solution $B$. To illustrate with an example, let us consider an arbitrary bi-objective optimization problem with five solutions in the objective space as shown in figure 3.1. We also assume that the goal of the problem is to maximize objective function $f_{1}$ and minimize objective function $f_{2}$.

If we do a domination comparison of solutions 1 and 2: assume the objective function evaluations in the form $\left(f_{1}, f_{2}\right)$ of both solutions are $(9,2)$ and $(8,5)$ respectively. We observe that solution 1 dominates solution 2 because: it has a higher objective 


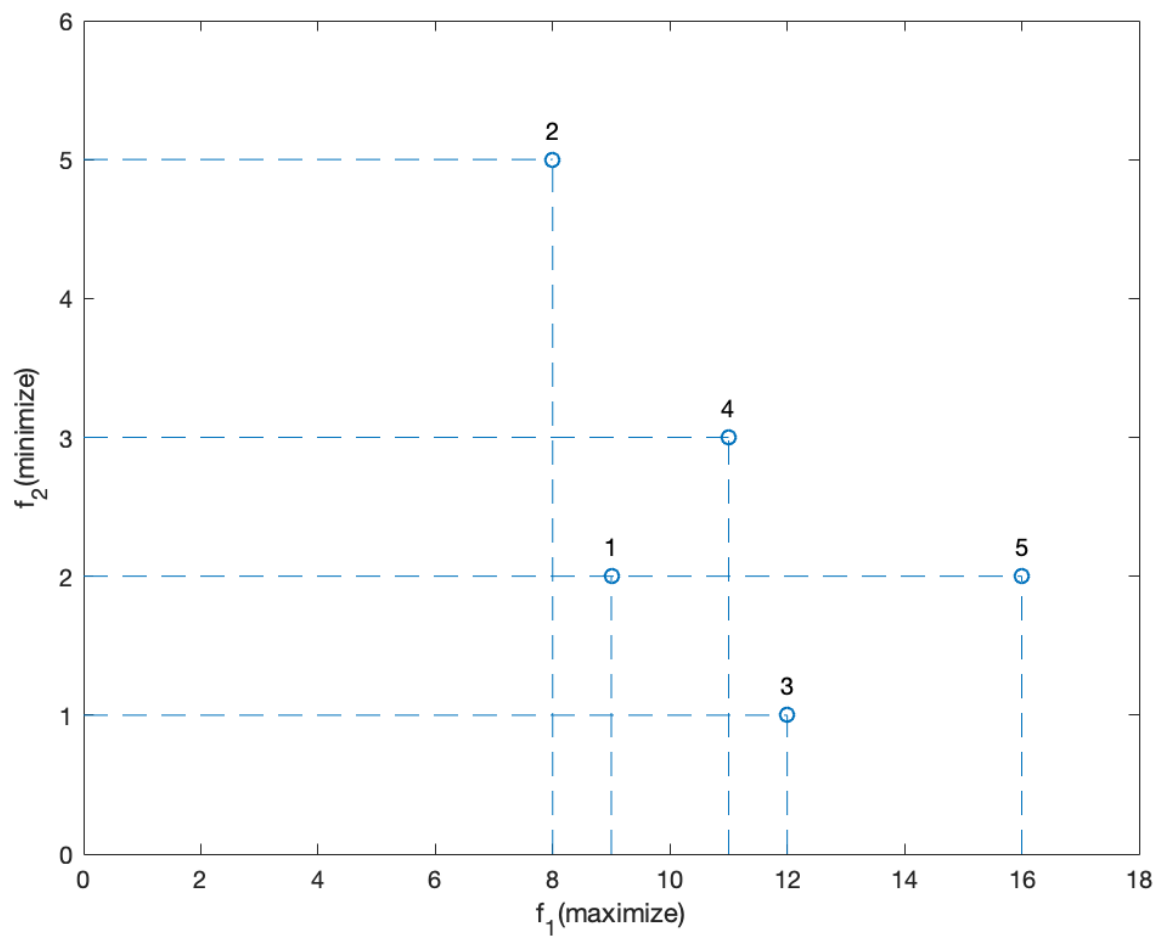

Figure 3.1: A sample decision space to illustrate dominance 
function value for the $f_{1}$ objective which needs to be maximized and a lower objective function value for the $f_{2}$ objective which needs to be minimized. Another instance is if we compare solutions 1 and 5 , we can categorically say that solution 5 dominates solution 1 even though they have the same $f_{2}$ objective function values, solution 5 has a better $f_{1}$ objective function value as stated in the second condition above.

Finally, if we compare compare solutions 1 and 4 to show an illustration of a pair of non-dominated solutions. We can observe from figure 3.1 that solution 1 has a better objective function value for the $f_{2}$ objective that needs to be minimized just the same way solution 4 has a better objective function value for the $f_{1}$ objective that needs to be maximized. Hence, a set of non-dominated solutions is a set of all the solutions that are not dominated by any member of the solution set. Therefore, solutions 3 and 5 in figure 3.1 are the non-dominated solutions.

\subsubsection{Pareto-Optimal Solution}

An interesting thing about multi-objective optimization problems is that solving them produces a set of non-dominated trade-off solutions in the feasible decision space and is known as a pareto-optimal set as shown in figure 3.2. The set of trade-off solutions are equally nondominated (i.e. non-inferior to one another), but might favour optimizing one of the objective functions over the others. The boundary formed by the set of solutions in the pareto-optimal set mapped from the feasible decision space to the feasible objective space is called the pareto-optimal front as depicted in figure 3.2.

One goal in solving a multi-objective optimization problem is to find a set of solutions as close as possible to the pareto-optimal front. Another is that the set of solutions be as diverse a possible. Diversity means that the solutions are uniformly spread out across the pareto-optimal front. As a step-up from single-objective genetic 


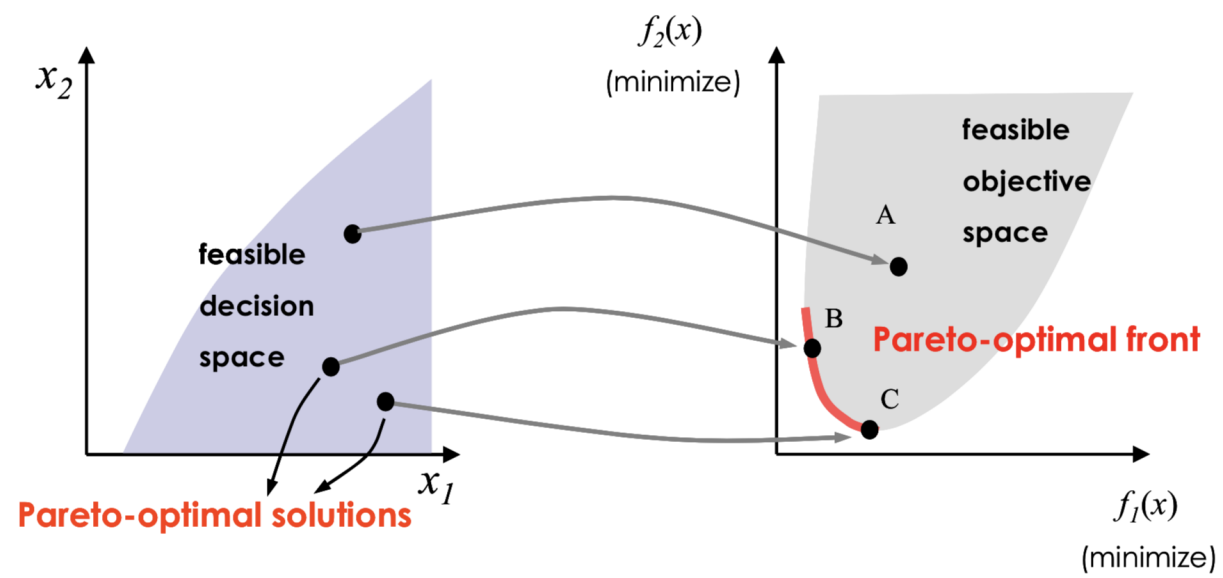

Figure 3.2: Graphical depiction of pareto-optimal set and pareto-optimal front

algorithms introduced in chapter 2, we will introduce multi-objective evolutionary algorithms (MOEAs) which are fast, elitist genetic algorithms. The algorithms show elitism by emphasizing the superior solutions in a population while locating paretooptimal, non-dominated solutions in multi-objective optimization problems.

These methods are suitable at inspecting trade-offs between multiple conflicting objectives to find multiple pareto-optimal solutions in one simulation run. This thesis focuses on four main algorithms which will be discussed in subsequent sections, namely: the elitist non-dominated sorting algorithm (NSGA-II), a reference pointbased non-dominated sorting algorithm (NSGA-III), an improved strength pareto evolutionary algorithm (SPEA2) and finally the pareto envelope-based selection algorithm (PESA-II). These are the most referenced and cited algorithms that have been proposed 


\subsection{A brief example}

As an example to illustrate the methods to be used in the thesis, the steps to solving the following relatively simple multi-objective problem in equation 3.2 will be thoroughly discussed. In this way, we can highlight the methods and differences between the competing algorithms to be evaluated. Furthermore, this is the first occurrence in the available literature where these algorithms are being evaluated at a detailed level. We will examine the effects of the specific differences in these algorithms.

\section{Objectives:}

$$
\begin{aligned}
& \min f_{1}(x)=x^{2} \\
& \min f_{2}(x)=(x-2)^{2}
\end{aligned}
$$

\section{Constraint:}

$$
-10 \leq x \leq 10 \text { and } x \in \mathbb{R}
$$

With this example, our goal is to find the optimum available solutions of the decision variable, $x$, capable of minimizing both objective functions while staying within the bounds of the constraint. Figure 3.3 shows a plot of both objective functions.

If we were to solve each objective function individually; the solution to function $f_{1}$ will be $x=0$ while the solution to $f_{2}$ will be $x=2$ as shown in figure 3.4 which shows a closer view of the plot in figure 3.3 with the $x$-axis limited to the range $[-1,3]$. In solving for both objectives simultaneously, we expect that a good multiobjective evolutionary algorithm will generate a pareto front formed by the decision variable values between $x=0$ and $x=2$ as illustrated by the shaded region in figure 3.4. Hence, solutions in the decision variable range $[0,2]$ constitute the set of pareto-optimal non-dominated solutions for the example problem in equation 3.2. 


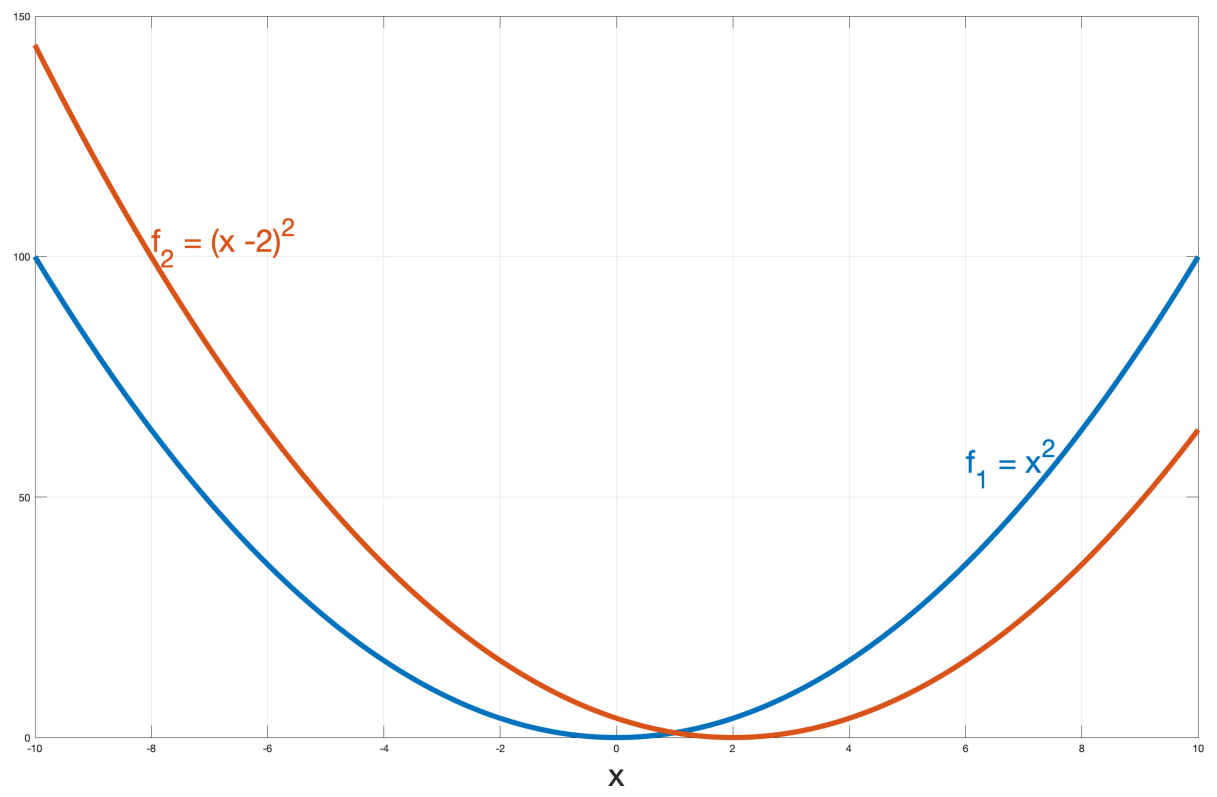

Figure 3.3: Graphical illustration of the objective functions in equation 3.2

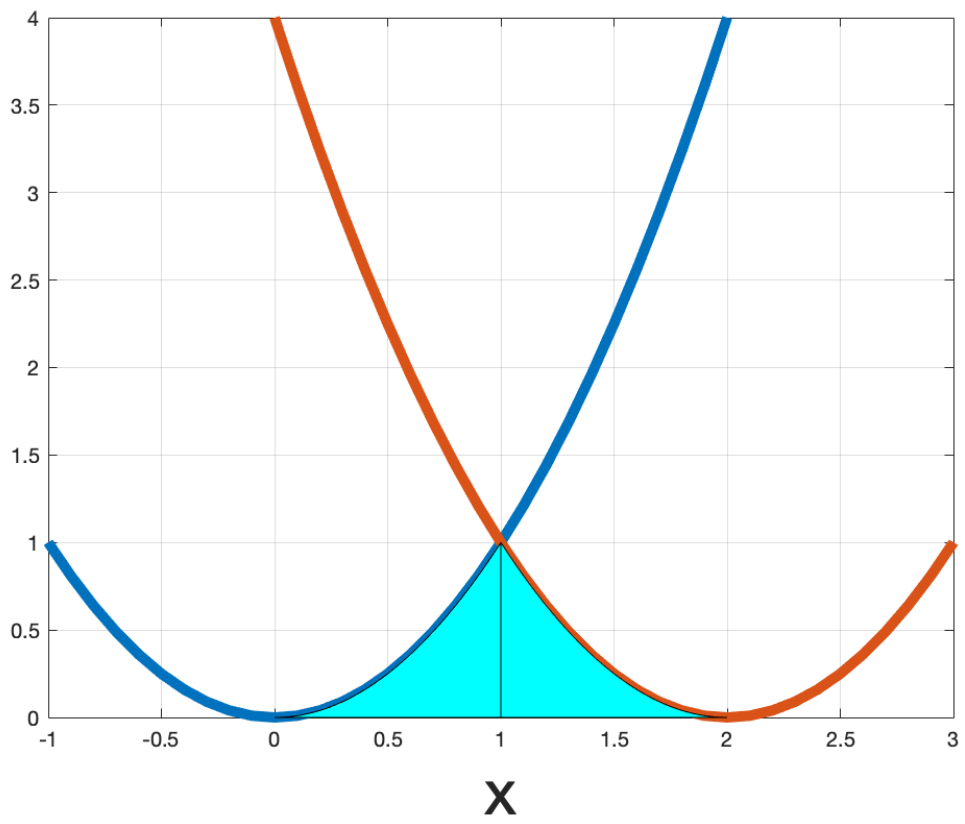

Figure 3.4: Closer view of the point at which the functions in equation 3.2 intercept 


\subsubsection{Elitist Non-dominated Sorting Genetic algorithm (NSGA-II)}

The elitist non-dominated sorting genetic algorithm, (NSGA-II), introduced by Deb, Kalyanmoy, et al in [28] is an evolutionary stochastic search algorithm for finding a multiple non-dominated set of solutions in multi-objective optimization problems. The algorithm emphasizes the principle of elitism which involves combining the parent and offspring populations and choosing the best solutions from the combined population with respect to their fitness and diversity values as shown in figure 3.5.

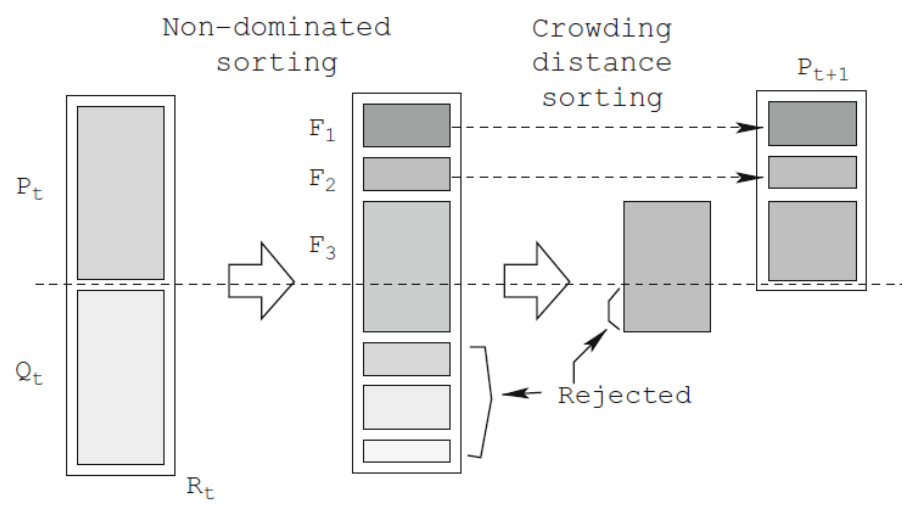

Figure 3.5: NSGA-II elitism approach [28]

The elitist approach of the algorithm is a good way of maintaining the best nondominated solutions at every generation to enhance the convergence of the algorithm. The algorithm also aims at exploring the search space through a diversity preservation mechanism. In the remainder of this section, we present the details of this algorithm: the fast non-dominated sorting procedure, the elitist preservation technique and the parameterless niching operator and apply it to solving the multi-objective optimization example in equation 3.2. 


\subsubsection{Initialization}

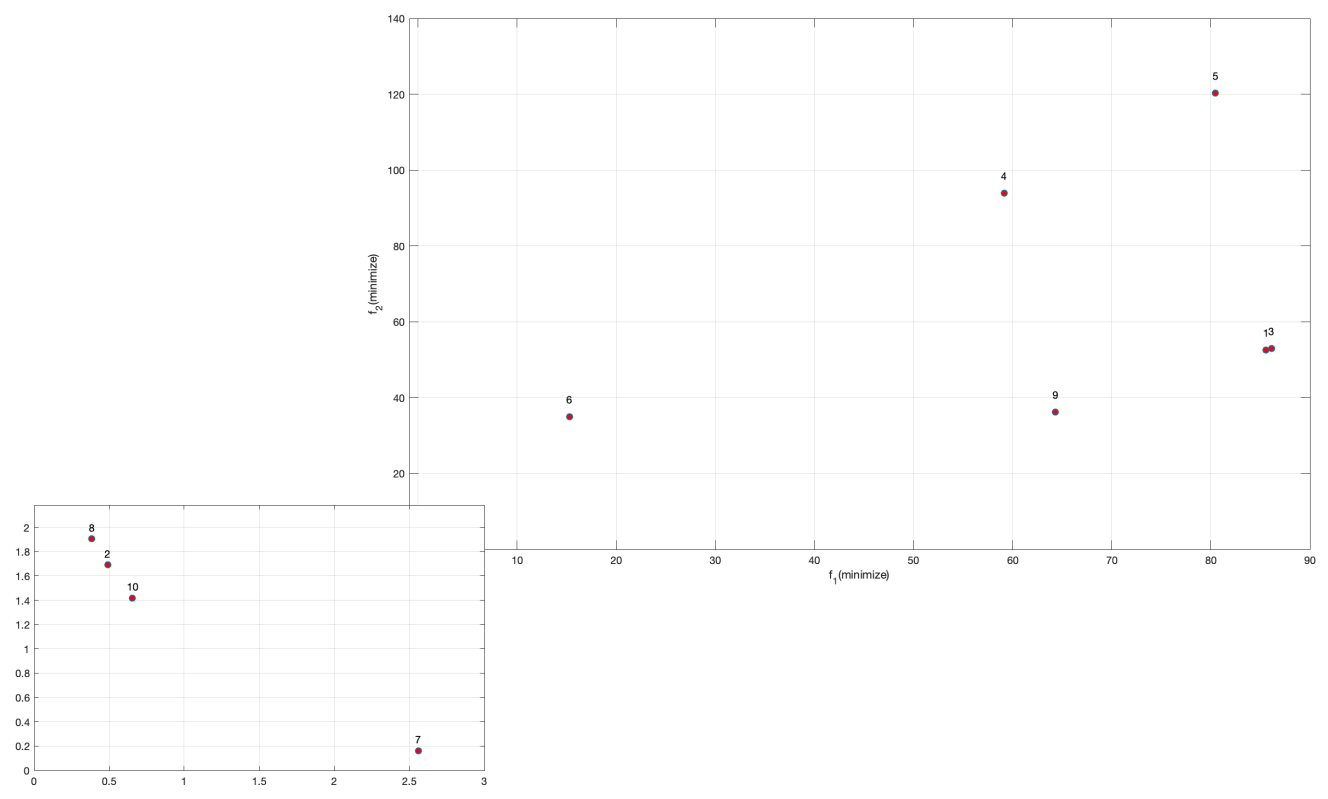

Figure 3.6: Plot of members of the parent population with the solutions close to the origin magnified

As with every other evolutionary genetic algorithm, the first step is to create an initial population, $P_{0}$, of size $N$, populated with randomly generated potential solutions depending on the type of encoding used as described in chapter 2. Since the decision variable $x$ is defined as a real-valued number, we use real-valued encoding for the decision variable $x$ in our solution. We are going to set the population size, $N=10$ so that it will be possible to highlight the differences between each one of the four algorithms.

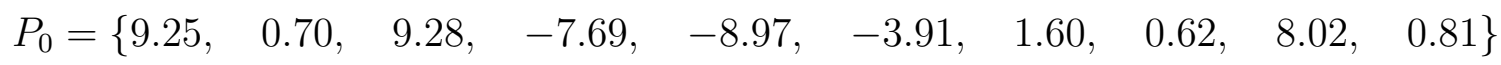

Equation 3.3 shows the randomly initialized parent population $P_{0}$ generated uniformly 
in the range $[-10,10]$. Figure 3.6 shows the solutions of the parent population plotted in an objective space. Equation 3.4 shows the function evaluations of the solutions in equation 3.3 in the form $\left(f_{1}, f_{2}\right)$; where $f_{1}=x^{2}$ and $f_{2}=(x-2)^{2}$.

$$
\begin{array}{rr}
F_{0}=\{(85.56,52.56), \quad(0.49,1.69), & (86.12,53.00), \\
(59.14,93.90), \quad(80.46,120.34), & (15.29,34.93), \\
(2.56,0.16), \quad(0.38,1.90), & (64.32,36.24),
\end{array}
$$

$(0.66,1.42)\}$

\subsubsection{Non-dominated sorting operation}

The parent population is then sorted into non-dominated fronts based on their assigned evaluated rank (or fitness) values representative of its non-domination position (e.g. rank 1 being the best, followed by ranks 2, 3 etc.). The fast non-dominated sorting operation of the NSGA-II algorithm is employed in sorting the solutions of the parent population. The initialized parent population in equation 3.3 is sorted using this approach as described in the following paragraph.

Initially, we define two parameters for each solution in the population, namely: the dominated count, $n_{p}$ and the domination set, $S_{p}$. The dominated count $n_{p}$, can be defined as the number of solutions that dominate solution $p$ while the domination set $S_{p}$, can be defined as a set containing solutions dominated by solution $p$. Both parameters (i.e. $n_{p}$ and $S_{p}$ ) are computed for each solution in the parent population.

To evaluate the values of both parameters, we first initialize the dominated count parameter of each solution to zero (i.e. $n_{p}=0$ ) and the domination set of each solution to an empty set (i.e. $S_{p}=\emptyset$ ). Hence, for the first solution (i.e. $P_{0}^{1}=9.25$ ) in equation 3.3 , we will initialize its dominated count parameter $n_{p}^{1}$ and its domination 
set parameter $S_{p}^{1}$ as shown in equation 3.5 below.

$$
\begin{array}{r}
\text { Solution 1: } 9.25 \\
n_{p}^{1}: 0 \\
S_{p}^{1}: \quad\{\}
\end{array}
$$

We then perform a dominance comparison of each solution with every other solution in the set to determine its dominated count $n_{p}$ and domination set $S_{p}$ values. Hence, if we consider again the first solution (i.e. Solution 1: $P_{0}^{1}=9.25$ ) in equation 3.3 and compare its objective function values (i.e. $F_{0}^{1}=(85.56,52.56)$ ) with that of the second solution in equation 3.3 (i.e. Solution 2: $P_{0}^{2}=0.70$ ) which has the function values $F_{0}^{2}=(0.49,1.69)$.

We can see that solution 2 dominates solution 1 because its function values are smaller when compared to those of solution 1 (since the objective of the problem is to minimize both objectives). Therefore, we increase the dominated count of solution 1 by one (i.e. $n_{p}^{1}=1$ ). Also, since solution 1 is dominated by solution 2 , we add the index number of solution 1 to the domination set parameter of solution 2 (i.e. $\left.S_{p}^{2}=\{1\}\right)$.

We proceed to perform the dominance comparison operation between each solution and every other solution in the set of equation 3.3. Hence, the dominated count $n_{p}$ and domination set $S_{p}$ parameters for the solutions in equation 3.3 are shown in table 3.1 below where Solution 1 is dominated by 6 solutions in the population set of equation 3.3 and it also dominates Solution 3. Similarly, Solution 2 is non-dominated by any solution in the population set of equation 3.3 and it dominates Solutions 1, 2, $4,5,6$ and 9 .

At this point, we take note of the solutions that have their dominated count 


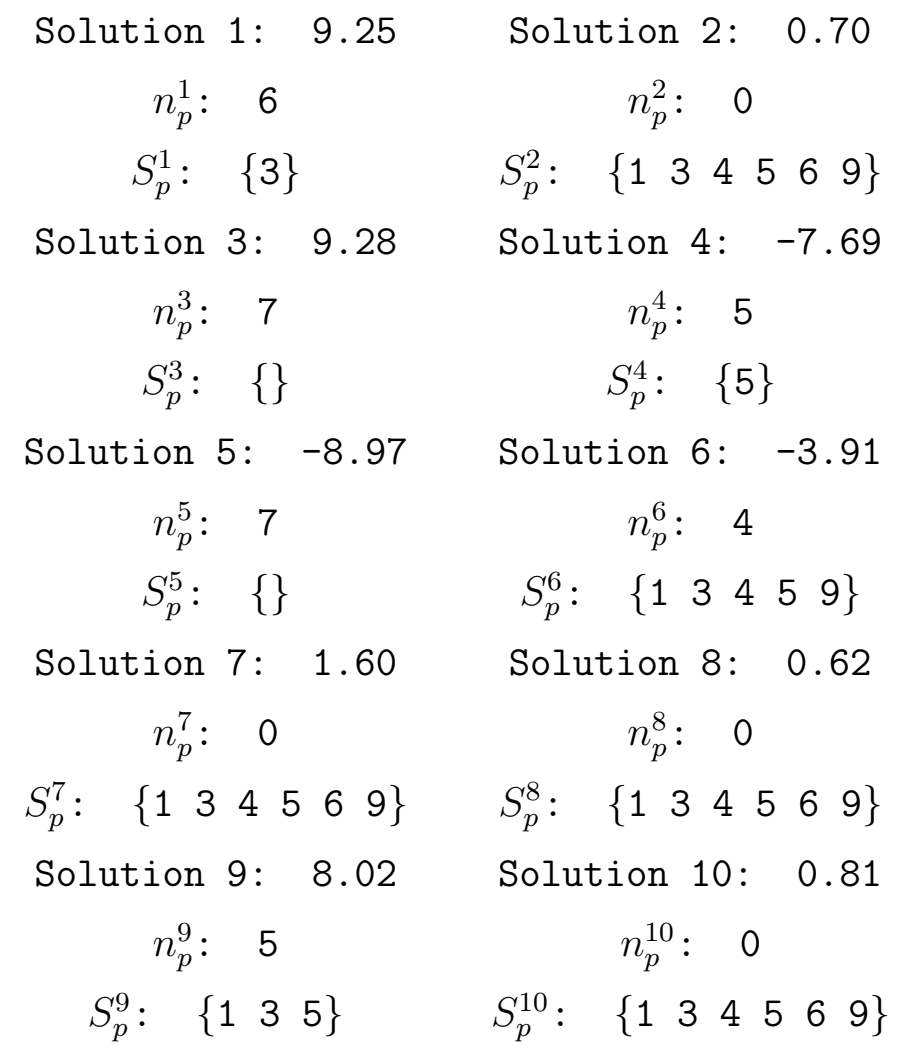

Table 3.1: Dominated count and domination set parameters 
$n_{p}=0$, (i.e. those that are not dominated by any solutions in the parent population set) and add them to the first non-dominated front, $\mathcal{F}_{1}$ as shown in equation 3.6. Hence, according to table 3.1, the solutions that will be in the first non-dominated front are Solutions 2, 7, 8 and 10 as shown in equation 3.6 below,

$$
\mathcal{F}_{1}=\{2, \quad 7, \quad 8, \quad 10\}
$$

where; $\mathcal{F}_{1}$ is the first non-dominated front set and its elements include: Solution $2=$ 0.70 , Solution $7=1.60$, Solution $8=0.62$ and Solution $10=0.81$ from the parent population set in equation 3.3.

The next step in the sorting process is the domination count reduction operation. For this operation, we loop through the solutions in the first (or current) non-dominated front; and for each solution, reduce the dominated count, $n_{p}$, of each solution in its domination set, $S_{p}$ by one unit. Hence, for the first solution in equation 3.6 i.e. $\mathcal{F}_{1}^{1}=$ Solution 2 (i.e. $P_{0}^{2}=0.70$ from the parent population in equation 3.3) whose domination set is given as: $S_{p}^{2}=\left\{\begin{array}{ll}1345 & 69\end{array}\right\}$ as shown in table 3.1. We then reduce the corresponding dominated counts $n_{p}$ of each solution in the set $S_{p}^{2}$ by one. Hence, Solution 1's dominated count (i.e. $n_{p}^{2}$ ) which was 6 in table 3.1 will now be equal to 5. Likewise, the dominated counts of Solutions 3, 4, 5, 6 and 9 will each be reduced by 1 ; hence, $n_{p}^{3}=6, n_{p}^{4}=4, n_{p}^{5}=6, n_{p}^{6}=3$ and $n_{p}^{9}=4$ respectively as shown in table 3.2 .

Likewise for the second solution in the first non-dominated set, $\mathcal{F}_{1}$ in equation 3.6 i.e. $\mathcal{F}_{1}^{2}=$ Solution 7 (i.e. $P_{0}^{7}=1.60$ from the parent population in equation 3.3) whose domination set is also given as: $S_{p}^{7}=\left\{\begin{array}{llllll}1 & 3 & 4 & 5 & 6 & 9\end{array}\right\}$ as shown in table 3.1. Again, we reduced the corresponding dominated counts $n_{p}$ of each solution in the set 


$\begin{array}{rr}\text { Solution } 1: 9.25 & \text { Solution } 3: 9.28 \\ n_{p}^{1}: 5 & n_{p}^{3}: 6 \\ S_{p}^{1}:\{3\} & S_{p}^{3}:\{\} \\ \text { Solution } 4: \quad-7.69 & \text { Solution } 5:-8.97 \\ n_{p}^{4}: 4 & n_{p}^{5}: 6 \\ S_{p}^{4}:\{5\} & S_{p}^{5}:\{\} \\ \text { Solution } 6:-3.91 & \text { Solution } 9: 8.02 \\ n_{p}^{6}: 3 & n_{p}^{9}: 4\end{array}$

Table 3.2: Updated dominated count of the solutions in solution 2's domination set

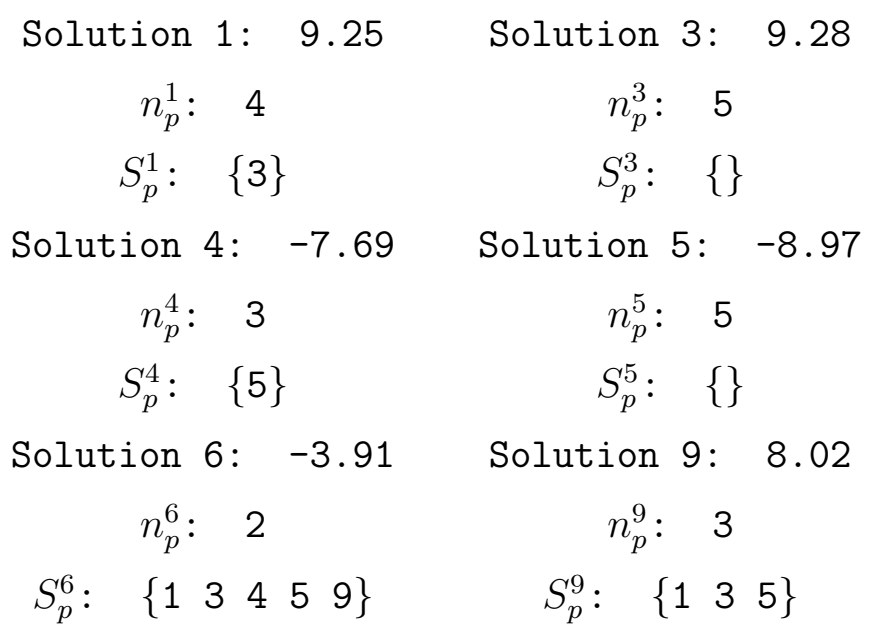

Table 3.3: Updated dominated count of the solutions in solution $7^{\prime}$ 's domination set 
$S_{p}^{7}$ by one as shown in table 3.3.

We repeat the same procedure for the remaining solutions in the first nondominated front i.e. $\mathcal{F}_{1}^{3}=$ Solution 8 and $\mathcal{F}_{4}^{2}=$ Solution 10 in equation 3.6. Since the solutions in the first non-dominated set have the same domination set (i.e. they all dominate the same set of solutions), the updated dominated count of the solutions in their domination sets after reducing their dominated counts is shown in table in 3.4 .

\begin{tabular}{|c|c|}
\hline $\begin{array}{c}\text { Solution 1: } 9.25 \\
n_{p}^{1}: 2\end{array}$ & $\begin{array}{c}\text { Solution } 3: 9.28 \\
n_{p}^{3}: \quad 3\end{array}$ \\
\hline$S_{p}^{1}: \quad\{3\}$ & $S_{p}^{3}: \quad\{\}$ \\
\hline $\begin{array}{c}\text { Solution } 4:-7.69 \\
n_{p}^{4}: \quad 1\end{array}$ & $\begin{array}{c}\text { Solution } 5: \quad-8.97 \\
n_{p}^{5}: \quad 3\end{array}$ \\
\hline$S_{p}^{4}: \quad\{5\}$ & $S_{p}^{5}: \quad\{\}$ \\
\hline Solution 6: $\quad-3.91$ & Solution 9: 8.02 \\
\hline $\begin{array}{llll} & n_{p}^{6}: & 0 & \\
S_{p}^{6}: & \left\{\begin{array}{lllll}1 & 3 & 4 & 5 & 9\end{array}\right\}\end{array}$ & 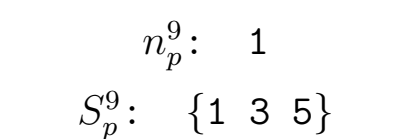 \\
\hline
\end{tabular}

Table 3.4: Updated dominated count and domination set parameters of the solutions in the first non-dominated set, $\mathcal{F}_{1}$

After going through all the solutions in the first (or current) non-dominated front and reducing the dominated counts of all the solutions in their corresponding domination sets, all solutions that now have their domination count, $n_{p}$, equal to zero will be added to the next non-dominated front. From table 3.4, we can see that only Solution 6 has its dominated count equal to zero; hence, it will be added to the next (i.e. the second) non-dominated front (or rank) as shown in equation 3.7.

$$
\mathcal{F}_{2}=\{6\}
$$


From equation $3.7, \mathcal{F}_{2}$ is the second non-dominated front and it contains only Solution $6=-3.91$ from the parent population in equation 3.3 as its only member. In a situation where after carrying out the dominated count reduction procedure, no solution in the domination sets has a domination count, $n_{p}=0$, we repeat the domination count reduction operation as described above.

We can see from the domination set, $S_{p}^{6}$ in table 3.4 that Solution 6 in the second non-dominated front, $\mathcal{F}_{2}$ dominates Solutions $1,3,4,5$ and 9 . To populate the next non-dominated front (i.e. the third non-dominated front, $\mathcal{F}_{3}$ ), we would reduce the corresponding domination counts, $n_{p}$ for Solutions $1,3,4,5$, and 9 by one unit each. Hence, the updated dominated counts for Solutions 1, 3, 4, 5, and 9 will be $n_{p}^{1}=1$, $n_{p}^{3}=2, n_{p}^{4}=0, n_{p}^{5}=2$ and $n_{p}^{9}=0$ respectively as shown in table 3.5. From table

\begin{tabular}{|c|c|}
\hline Solution 1: 9.25 & Solution 3: 9.28 \\
\hline$n_{p}^{1}: \quad 1$ & $n_{p}^{3}: \quad 2$ \\
\hline$S_{p}^{1}: \quad\{3\}$ & $S_{p}^{3}: \quad\{\}$ \\
\hline $\begin{array}{c}\text { Solution } 4:-7.69 \\
n_{p}^{4}: 0\end{array}$ & $\begin{array}{c}\text { Solution } 5:-8.97 \\
n_{p}^{5}: 2\end{array}$ \\
\hline$S_{p}^{4}: \quad\{5\}$ & $S_{p}^{5}: \quad\{\}$ \\
\hline $\begin{array}{c}\text { Solution 9: } 8.02 \\
n_{p}^{9}: \quad 0\end{array}$ & \\
\hline$S_{p}^{9}: \quad\left\{\begin{array}{lll}1 & 3 & 5\end{array}\right\}$ & \\
\hline
\end{tabular}

Table 3.5: Updated dominated count and domination set parameters of the solution in the second non-dominated set, $\mathcal{F}_{2}$

3.5, Solutions 4 and 9 now have their dominated count values equal to zero (i.e. $\left.n_{p}^{4}=n_{p}^{9}=0\right)$. Therefore, Solutions 4 and 9 will constitute the members of the third 
non-dominated front as shown in equation 3.8.

$$
\mathcal{F}_{3}=\{4, \quad 9\}
$$

To populate the next non-dominated front (i.e. the fourth non-dominated front, $\mathcal{F}_{4}$ ), we once again look at the corresponding domination sets of the solutions in our current non-dominated front (i.e. the third non-dominated front $\mathcal{F}_{3}$ ). From the domination set, $S_{p}^{4}$ in table 3.5, Solution 4 dominates only Solution 5; hence we decrease Solutions 5's dominated count by 1 thereby making its dominated count, $n_{p}^{5}=1$. Moving on to the next solution in $\mathcal{F}_{3}$ (i.e. Solution 9 in equation 3.7), we can see from $S_{p}^{9}$ in table 3.5 that Solution 9 dominates Solutions 1, 3 and 5 . Once again, we decrement the the corresponding dominated count values, $n_{p}$ of Solutions 1,3 and 5 to give $n_{p}^{1}=0, n_{p}^{3}=1$ and $n_{p}^{5}=0$ respectively as shown in table 3.6.

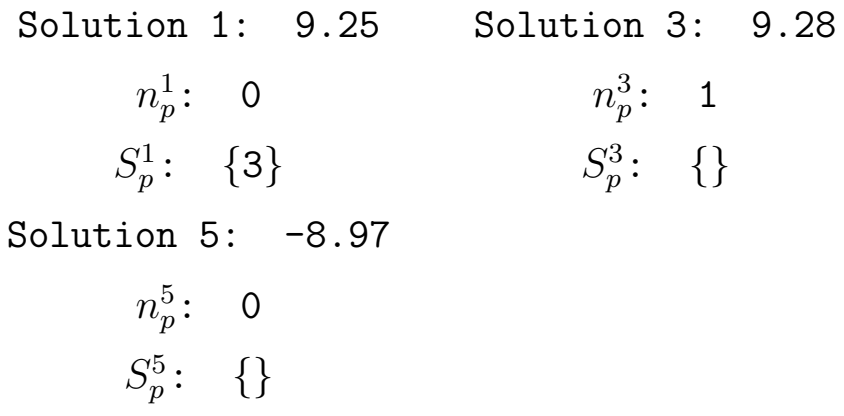

Table 3.6: Updated dominated count and domination set parameters of the solutions in the third non-dominated set, $\mathcal{F}_{3}$

From table 3.6, we can see that Solutions 1 and 5 now have updated dominated count values, $n_{p}=0$. Hence, Solutions 1 and 5 will be added to the next nondominated front (i.e. the fourth non-dominated front, $\mathcal{F}_{4}$ as shown in equation 3.9.

$$
\mathcal{F}_{4}=\{1, \quad 5\}
$$


Finally, to populate the next non-dominated front (i.e. the fifth non-dominated front, $\mathcal{F}_{5}$ ), we will again look at the corresponding domination sets, $S_{p}$ of Solutions 1 and 5 in equation 3.9. From $S_{p}^{1}$ and $S_{p}^{5}$ in table 3.6, we can see that Solution 1 dominates only Solution 3 and Solution 5 has an empty domination set. Hence, have to reduce only the dominated count, $n_{p}$ of Solution 3 in table 3.6 by one unit resulting in $n_{p}^{3}=0$ as shown in table 3.7 .

Solution $3: 9.28$
$n_{p}^{3}: 0$
$S_{p}^{3}:\{\}$

Table 3.7: Updated dominated count and domination set parameters of the solutions in the fourth non-dominated set, $\mathcal{F}_{4}$

From table 3.7, the dominated count for Solution $3, n_{p}^{3}=0$, hence, we add Solution 3 to the fifth non-dominated front, $\mathcal{F}_{5}$ as shown in equation 3.10. Since the corresponding domination set, $S_{p}^{3}$ of Solution 3 in table 3.7 is an empty set (i.e. it has no solutions it dominates), we stop the non-domination sorting algorithm. Therefore, the solutions in the parent population in equation 3.3 are ranked into the following 5 non-dominated fronts:

$$
\begin{aligned}
& \mathcal{F}_{1}=\{2, \quad 7, \quad 8, \quad 10\} \\
& \mathcal{F}_{2}=\{6\} \\
& \mathcal{F}_{3}=\{4, \quad 9\} \\
& \mathcal{F}_{4}=\{1, \quad 5\} \\
& \mathcal{F}_{5}=\{3\}
\end{aligned}
$$

The pseudo code in algorithm 2 a summary of the fast and elitist non-dominated 


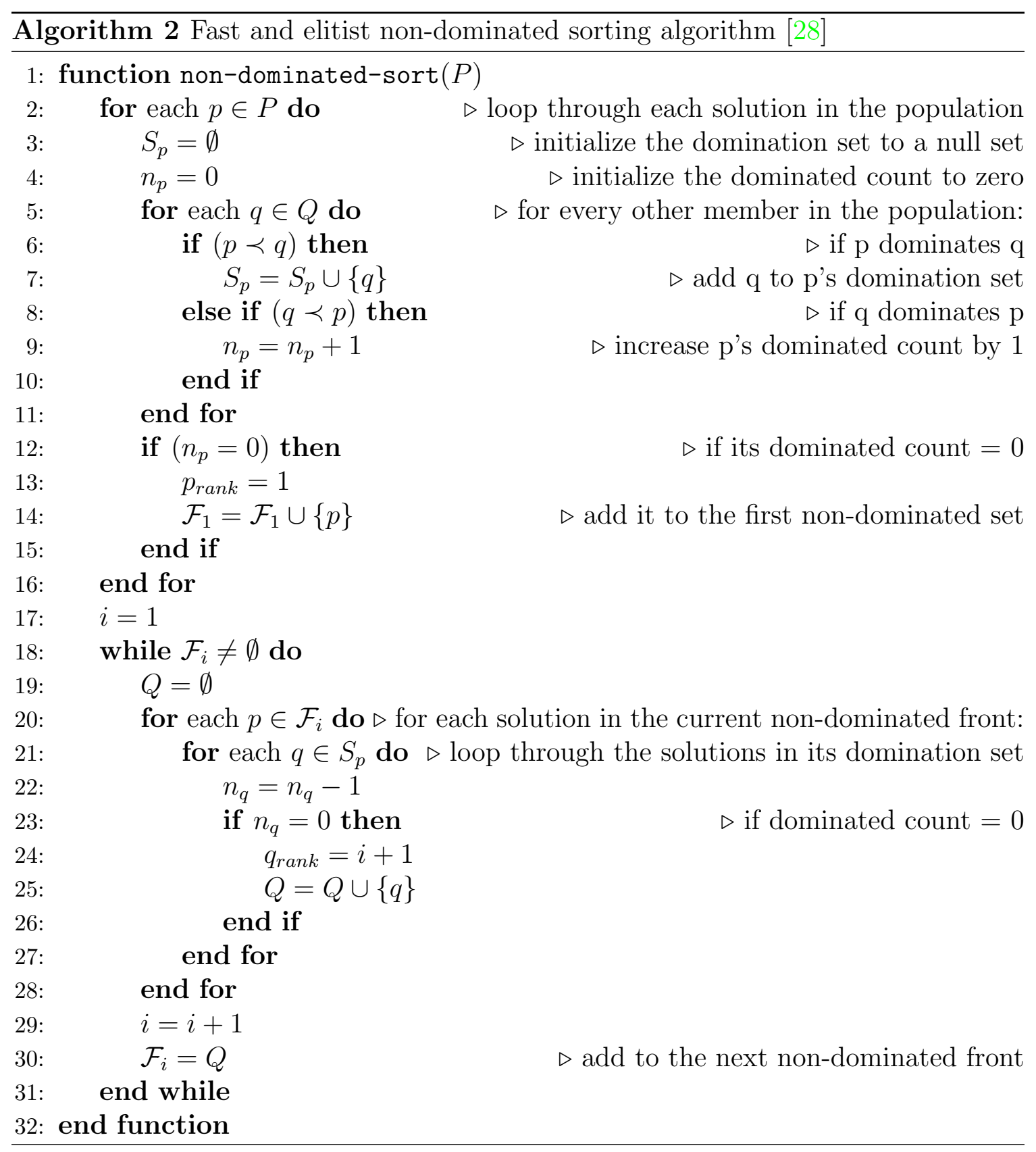


sorting algorithm.

\subsubsection{Crowding distance assignment operation}

We compute the value of the crowding-distance parameter, $I_{D}$ for the solutions in each non-dominated front. The crowding-distance value represents an estimate of the density of solutions surrounding a particular solution in the population. It is evaluated by computing the average distance of the adjacent solutions on either side of a solution in the population along each objective.

The first step in computing the crowding-distance value for the solutions in a nondominated front is to sort the population in the non-dominated front, $\mathcal{F}_{i}$ according to each objective function value in ascending order of magnitude. This implies that we will have the population sorted in $M$ ways (where $M$ is the number of objectives). For our example problem, evaluating the solutions in the first non-dominated front, $\mathcal{F}_{1}$ (i.e. $\mathcal{F}_{1}=\{2, \quad 7, \quad 8, \quad 10\}$ in equation 3.6) along each objective is shown in equation 3.11:

$$
\begin{aligned}
& f_{1}(x)=\{0.49, \quad 2.56, \quad 0.38, \quad 0.66\} \\
& f_{2}(x)=\{1.69, \quad 0.16, \quad 1.90, \quad 1.42\}
\end{aligned}
$$

where the $f_{1}(x)$ and $f_{2}(x)$ arrays contains the first and second objective function (i.e. $f_{1}(x)=x^{2}$ and $\left.f_{2}(x)=(x-2)^{2}\right)$ evaluations of the solutions in the first nondominated front, $\mathcal{F}_{1}$ in equation 3.6. Equation 3.12 shows the objective function solution arrays in equation 3.11 sorted in ascending order of magnitude.

$$
\begin{aligned}
& f_{1}^{\text {sorted }}(x)=\left\{\begin{array}{llll}
0.38, & 0.49, & 0.66, & 2.56
\end{array}\right\} \\
& f_{2}^{\text {sorted }}(x)=\left\{\begin{array}{llll}
0.16, & 1.42, & 1.69, & 1.90
\end{array}\right\}
\end{aligned}
$$


Next, we defined two crowding distance parameter sets, $\left(I_{D 1}\right)$ and $\left(I_{D 2}\right)$ for each objective function (i.e. $f_{1}(x)$ and $f_{2}(x)$ respectively). The two crowding distance parameter sets will have the same size as the non-dominated front set (i.e. $N=4$ ) as shown in equation 3.13:

$$
\begin{aligned}
& I_{D 1}=\left\{I_{D 1}^{1}, \quad I_{D 1}^{2}, \quad I_{D 1}^{3}, \quad I_{D 1}^{4}\right\} \\
& I_{D 2}=\left\{I_{D 2}^{1}, \quad I_{D 2}^{2}, \quad I_{D 2}^{3}, \quad I_{D 2}^{4}\right\}
\end{aligned}
$$

where $I_{D j}^{i}$ corresponds to the crowding distance value for solution $i$ in the first (or current) non-dominated front along the $j^{\text {th }}$ objective. For example, $I_{D 1}^{1}$ corresponds to the crowding distance value for Solution 2 in the first non-dominated front, $\mathcal{F}_{1}$ in equation 3.6 along the first objective function, $f_{1}(x)$ in equation 3.2 .

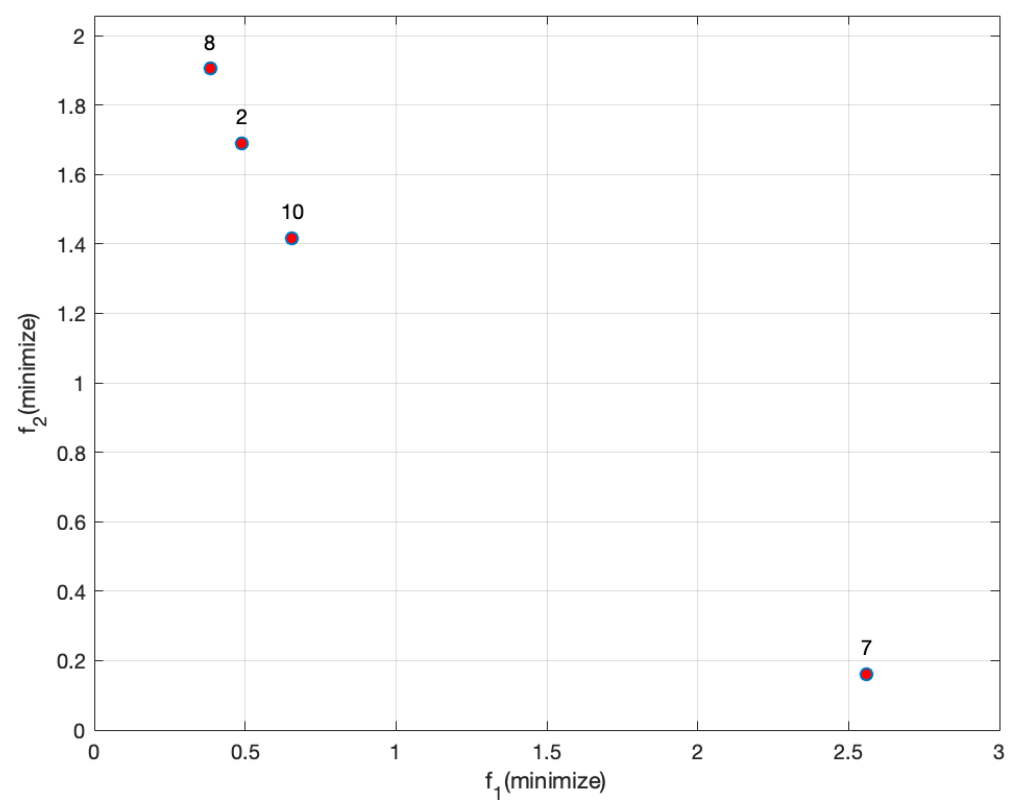

Figure 3.7: Pareto front for the first non-dominated front

The next thing is to set the the crowding-distance values for the boundary solutions (i.e. solutions with the smallest and largest function values) along each objective 
to infinity $(\infty)$. From equation 3.12 , the boundary solutions along the first objective, $f_{1}^{\text {sorted }}(x)$ are 0.38 and 2.56 which correspond to Solution 8 and Solution 7 respectively in equation 3.6 (also depicted graphically in figure 3.7). Therefore, we set the crowding distance values for the boundary solutions to $\infty$. We have $I_{D 1}^{3}=\infty$ and $I_{D 1}^{2}=\infty$ as shown in equation 3.14 ;

$$
\begin{aligned}
& I_{D 1}=\left\{I_{D 1}^{1}, \quad \infty, \quad \infty, \quad I_{D 1}^{4}\right\}
\end{aligned}
$$

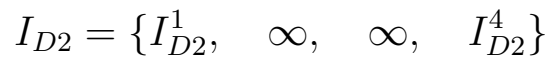

where $I_{D 1}^{3}$ corresponds to Solution $8=0.38$ and $I_{D 1}^{2}$ corresponds to Solution $7=$ 2.56. Likewise, the boundary solutions for the second objective, $f_{2}(x)$ are 0.16 and 1.90 which correspond to Solutions 7 and 8 respectively as shown in figure 3.7. Hence, their crowding distance values $I_{D 2}^{2}=\infty$ and $I_{D 2}^{3}=\infty$ as shown in equation 3.14.

Consequently, all other intermediate solutions (i.e. solutions 2 to $(N-1)$ ) in the current non-dominated front; where $\mathrm{N}$ is the number of solutions in the front are assigned crowding-distance values equal to the absolute normalized difference in the objective function values of its two adjacent solutions computed as shown in equation 3.15 .

$$
\begin{aligned}
& I_{D}=\frac{\left|\mathcal{I}\{i+1] \cdot m-f_{m}^{\text {min }}\right|}{\left|f_{m}^{\text {max }}-f_{m}^{\text {min }}\right|}-\frac{\left|\mathcal{I}\{i-1] \cdot m-f_{m}^{\text {min }}\right|}{\left|f_{m}^{\text {max }}-f_{m}^{\text {min }}\right|} \\
& I_{D}=\frac{|\mathcal{I}\{i+1] \cdot m-\mathcal{I}\{i-1] \cdot m|}{\left|f_{m}^{\text {max }}-f_{m}^{\text {min }}\right|}
\end{aligned}
$$

where $\mathcal{I}\{i] . m$ refers to the $m$ th objective function value of the $i$ th solution in the sorted set $\mathcal{I}$ and $f_{m}^{\text {min }}$ and $f_{m}^{\text {max }}$ are the minimum and maximum values along the $m$ th objective function in the non-dominated front.

Therefore, the crowding distance value computation for $I_{D 1}^{1}$ which corresponds to 
the first solution (Solution 2) in the first non-dominated front in equation 3.6 along the first objective (i.e. $f(x)=x^{2}$ ) is detailed in equation 3.16;

$$
\begin{aligned}
& I_{D 1}^{1}=\frac{|0.66-0.38|}{|2.56-0.38|} \\
& I_{D 1}^{1}=0.13
\end{aligned}
$$

where 0.66 is gotten from the first sorted set, $f_{1}^{\text {sorted }}$ in equation 3.12 and corresponds to $\mathcal{I}[i+1] . m$ in equation 3.15 along the first objective (i.e. $m=1$ ); 0.38 also gotten from $f_{1}^{\text {sorted }}$ in equation 3.12 corresponds to $\mathcal{I}[i-1] . m$ in equation 3.15. Finally, the values 0.38 and 2.56 correspond to the $f_{m}^{\min }$ and $f_{m}^{\max }$ variables gotten from equation 3.12 .

Likewise, the crowding distance value computation for $I_{D 1}^{4}$ which corresponds to Solution 10 in the first non-dominated front in equation 3.6 is shown in equation 3.17 below:

$$
\begin{aligned}
& I_{D 1}^{4}=\frac{|2.56-0.49|}{|2.56-0.38|} \\
& I_{D 1}^{4}=0.95
\end{aligned}
$$

where 2.56 from $f_{1}^{\text {sorted }}$ in equation 3.12 corresponds to $\mathcal{I}[i+1] . m(m=1)$ in equation $3.15,0.49$ corresponds to $\mathcal{I}[i-1] . m$ in equation 3.15 and the denominator values (i.e. 2.56 and 0.38 ) correspond to the $f_{m}^{\max }$ and $f_{m}^{\min }$ variables in equation 3.15. Similarly, the computation for the unknown crowding distance values (i.e. $I_{D 2}^{4}$ and $I_{D 2}^{1}$ ) along 
the second objective (i.e. $m=2$ ) is shown in equation 3.18 below.

$$
\begin{aligned}
& I_{D 2}^{4}=\frac{|1.69-0.16|}{|1.90-0.16|} \\
& I_{D 2}^{4}=0.88 \\
& I_{D 2}^{1}=\frac{|1.90-1.42|}{|1.90-0.16|} \\
& I_{D 2}^{1}=0.28
\end{aligned}
$$

where $I_{D 2}^{4}$ corresponds to crowding distance value of Solution 10 in the first nondominated front in equation 3.6 along the second objective, $I_{D 2}^{1}$ corresponds to the crowding distance value of Solution 2 also along the second objective and all the values are gotten from the sorted second set, $f_{2}^{\text {sorted }}$ in equation 3.12. Equation 3.19 shows the crowding distance values along both objectives.

$$
\begin{aligned}
& I_{D 1}=\{0.13, \quad \infty, \quad \infty, \quad 0.95\} \\
& I_{D 2}=\{0.28, \quad \infty, \quad \infty, \quad 0.88\}
\end{aligned}
$$

Finally the overall crowding-distance value for each solution in the first nondominated population in equation 3.6 is the sum of the individual crowding distance values along each objective; hence the total crowding distance value for the solutions in the first non-dominated front is given in equation 3.20. The crowding distance assignment operation is summarized in algorithm 3 .

$$
I_{D}=\{0.41, \quad \infty, \quad \infty, 1.83\}
$$




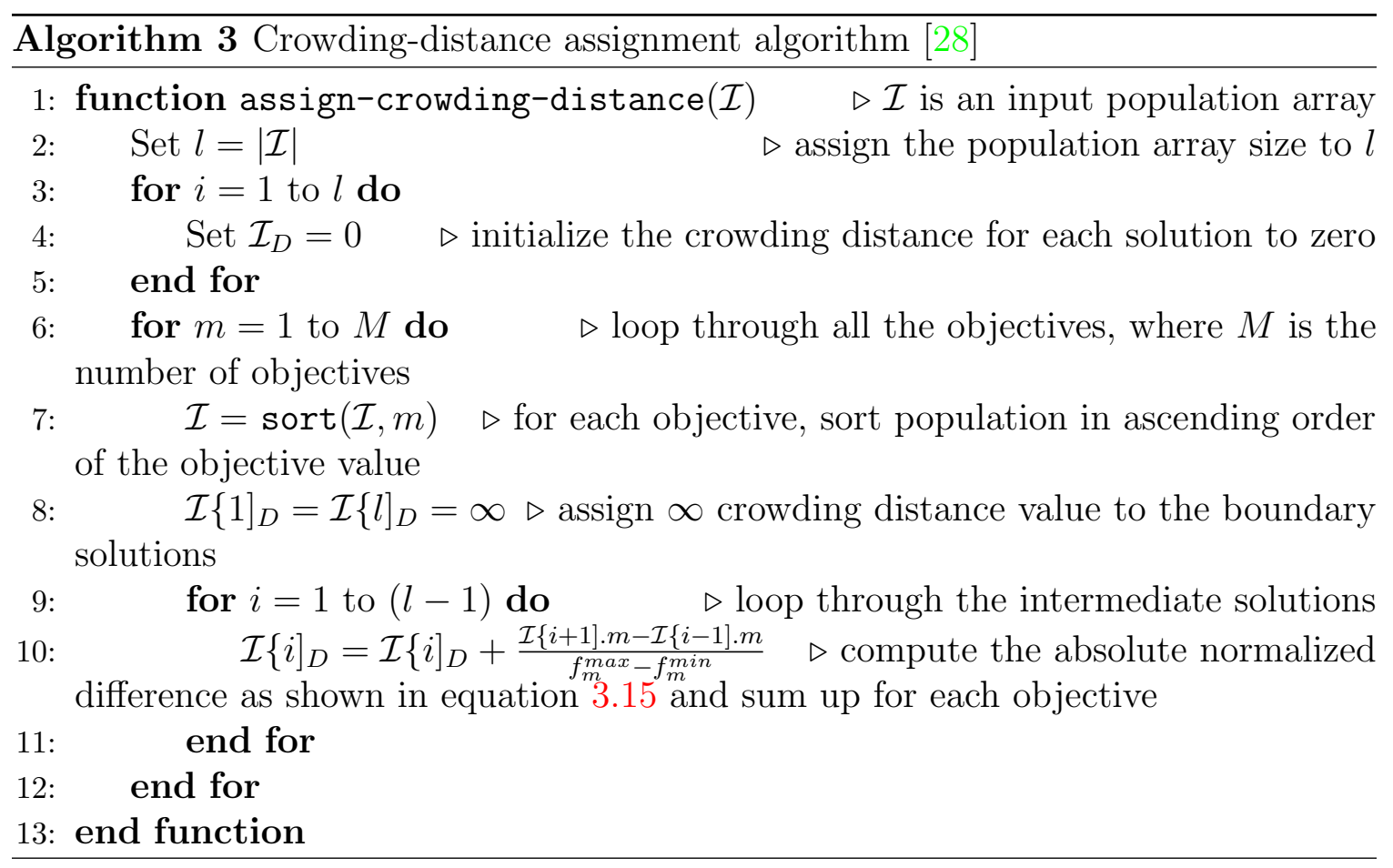

\subsubsection{Create offspring population set}

After the non-dominated sorting and crowding distance assignment procedures, the first generation offspring population, $Q_{0}$, also of size $N$, is then created from the initialized parent population using the usual genetic algorithm operators: selection, recombination and mutation as described in chapter 2. The selection operation is now based on the crowded-comparison operator $\left(\prec_{n}\right)$.

The crowded-comparison operator combines the non-domination $\operatorname{rank}\left(\mathcal{F}_{R}\right)$ and crowding-distance $\left(I_{D}\right)$ parameters to guide the selection operation process towards attaining a uniformly spread pareto-optimal front. Therefore, solution $i$ is selected over solution $j$ (i.e. $i \prec_{n} j$ ) if:

$$
\begin{aligned}
& \text { if }\left(\mathcal{F}_{R}(i)<\mathcal{F}_{R}(j)\right) \\
& \text { or } \\
& \quad \text { if }\left(\mathcal{F}_{R}(i)=\mathcal{F}_{R}(j)\right) \text { and }\left(I_{D}(i)>I_{D}(j)\right)
\end{aligned}
$$


Therefore, we prefer the solution with the lower (better) rank (non-domination front). Otherwise, if both solutions have the same rank, the one with larger crowdingdistance value is preferred. For example, if we apply the binary tournament selection algorithm to the parent population $P_{0}$ in equation 3.3 and it randomly picks solutions $P_{0}^{2}=0.70$ and $P_{0}^{7}=1.60$ which correspond to Solution 2 and Solution 7 in the first non-dominated front in equation 3.6. Comparing the solutions, first comparing their domination ranks (which is equal) since they belong to the same front; we then compare their crowding distance values in equation 3.20 (i.e. $I_{D} 1=0.41$ and $I_{D} 2=\infty$ respectively). Since $\infty$ is greater, we select $P_{0}^{7}=1.60$ as the parent solution. Applying the remaining genetic operators (i.e. recombination and mutation introduced and discussed in chapter 2) produces the offspring population in equation 3.22 .

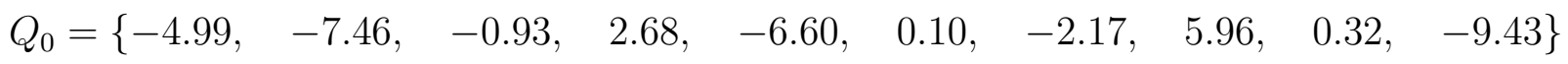

\subsubsection{Evolve next parent population}

Thereafter, both generations (the parent and the offspring) are combined to form an aggregated population, $R_{t}$, of size $2 N$ as shown in equation 3.23. Non-dominated sorting is once again applied to the combined population, $R_{t}$, to classify the solutions into ranked pareto-fronts. The sorting of the combined population, $R_{t}$, emphasizes the elitism concept of the algorithm stated earlier. This is because comparing solutions from the parent (which contained the previous optimal solutions) and offspring 
populations produces the superior solutions in each.

$$
\begin{aligned}
& R_{t}=\{9.25, \quad 0.70, \quad 9.28, \quad-7.69, \quad-8.97, \quad-3.91, \quad 1.60, \quad 0.62, \quad 8.02, \quad 0.81, \\
& -4.99, \quad-7.46, \quad-0.93, \quad 2.68, \quad-6.60, \quad 0.10, \quad-2.17, \quad 5.96, \quad 0.32, \quad-9.43\}
\end{aligned}
$$

To evolve the next generation, $P_{t+1}$, of size $N$, we extract, from the sorted combined population, the elite solutions. To do this, we once again perform nondomination sorting (as explained in section 3.3.1.2) on the combined population in order to group the solutions into non-dominated fronts (or ranks), where the first non-domination front contains the best solutions in the combined population as the set $\mathcal{F}_{1}$, the second as $\mathcal{F}_{2}$, third as $\mathcal{F}_{3}$ and so on.

$$
\begin{aligned}
& \mathcal{F}_{1}=\left\{\begin{array}{ll}
2, \quad 7, \quad 8, \quad 10, & 16,19
\end{array}\right\} \\
& \mathcal{F}_{2}=\left\{\begin{array}{ll}
14, & 13
\end{array}\right\} \\
& \mathcal{F}_{3}=\left\{\begin{array}{ll}
17, & 18
\end{array}\right\} \\
& \mathcal{F}_{4}=\{6\} \\
& \mathcal{F}_{5}=\left\{\begin{array}{ll}
9, & 11
\end{array}\right\} \\
& \mathcal{F}_{6}=\left\{\begin{array}{ll}
1, & 15
\end{array}\right\} \\
& \mathcal{F}_{7}=\{3,12\} \\
& \mathcal{F}_{8}=\{4\} \\
& \mathcal{F}_{9}=\{5\} \\
& \mathcal{F}_{10}=\{20\}
\end{aligned}
$$

The non-dominated fronts for the combined population $R_{t}$ is shown in equation 3.24; where the the first non-dominated front $\mathcal{F}_{1}$ consists Solutions 2, 7, 8, 10, 16 and 19 
from the combined population $R_{t}$ in equation 3.23. As we did before for the parent population, we will also compute and assign crowding distance values to the solutions in each non dominated front.

Since the population size of $R_{t}>P_{t+1}$ (i.e. $2 N>N$ ), hence not all the solutions in the combined population, $R_{t}$ will make it into the new generation. Consequently, if the size of $\mathcal{F}_{1}$ is less than that of $N$ (i.e. $\left|\mathcal{F}_{1}\right|<N$ ); where $|\cdot|$ denotes the cardinality of the set. All the solutions in $\mathcal{F}_{1}$ are chosen to populate the new generation, $P_{t+1}$. Likewise, if $\left|\mathcal{F}_{2}\right|<\left(N-\left|\mathcal{F}_{1}\right|\right)$, all the solutions in $\mathcal{F}_{2}$ will be added to the new generation. This continues till the members of the new population, $P_{t+1}$ are filled.

$$
P_{t+1}=\left\{\begin{array}{llllllllll}
0.70, & 1.60, & 0.62, & 0.81, & 0.10, & 0.32, & 2.68, & -0.93, & -2.17, & 5.96
\end{array}\right\}
$$

Therefore for our example problem, $N=10$ hence, only the solutions in the first $\left(\mathcal{F}_{1}\right)$, second $\left(\mathcal{F}_{2}\right)$ and third $\left(\mathcal{F}_{3}\right)$ non-dominated fronts will make it into the next generation as shown in equation 3.25.

If we encounter a situation where the last non-dominated front, $\mathcal{F}_{l}$ (to be added to the new generation's population set, $\left.P_{t+1}\right)$, contains more solutions than the spaces left to fill the new population (i.e. $\left|\mathcal{F}_{l}\right|>\left(|N|-\left|\mathcal{F}_{1} \cup \ldots \cup \mathcal{F}_{l-1}\right|\right)$ ). To keep the next generation's population size at $N$ and preserve diversity of the solutions by ensuring a uniform spread of solutions in the pareto-front, we sort the last non-dominated front using the crowding-distance values in descending order of magnitude and choose the set of solutions needed to fill the new generation.

Finally, we will repeat the algorithm procedures until our termination criteria is met. Figure 3.8 shows the pareto-optimal front for the solution to the example problem in equation 3.2, where the population size $N=100$ and the algorithm terminated after 100 iterations. In figure 3.8, the axes represent the two objective 
function values in the multi-objective problem in equation 3.2 and the points on the graph represent solutions in the final population. The NSGA-II algorithm is summarized in algorithm 4 below. The algorithm has a computational complexity of $O\left(M N^{2}\right)$, where $M$ is the number of objectives and $N$ is the population size.

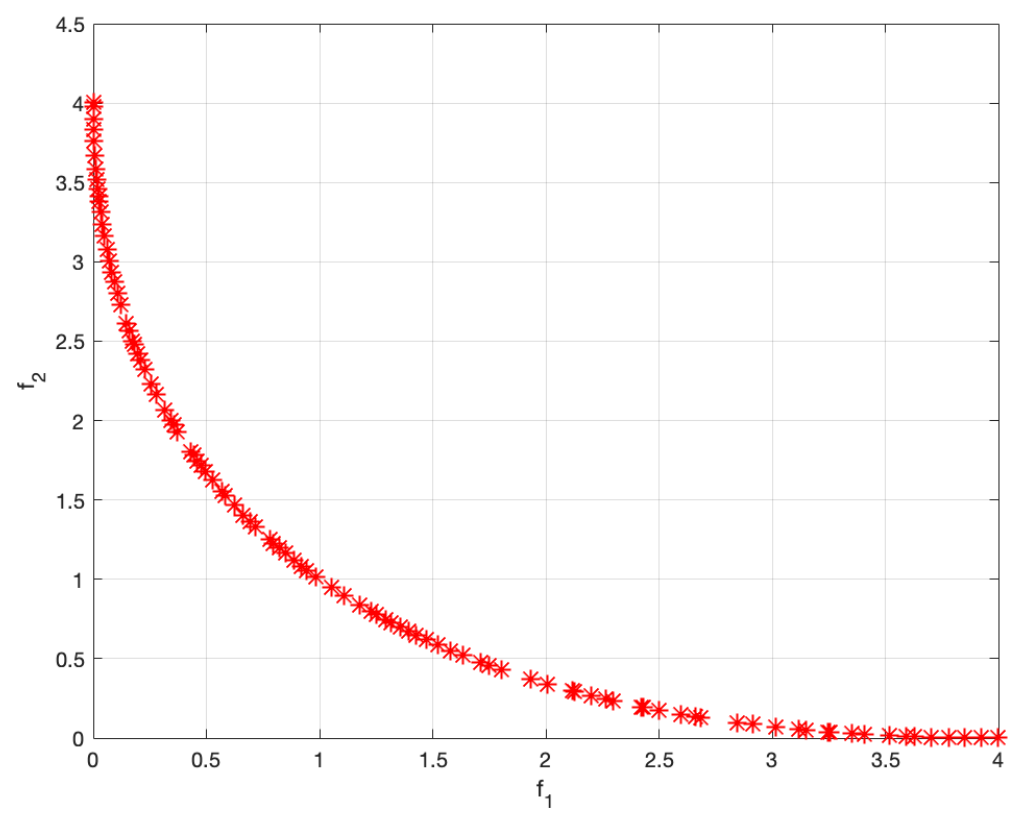

Figure 3.8: Pareto-optimal front showing solution to equation 3.2 using the NSGAII algorithm 


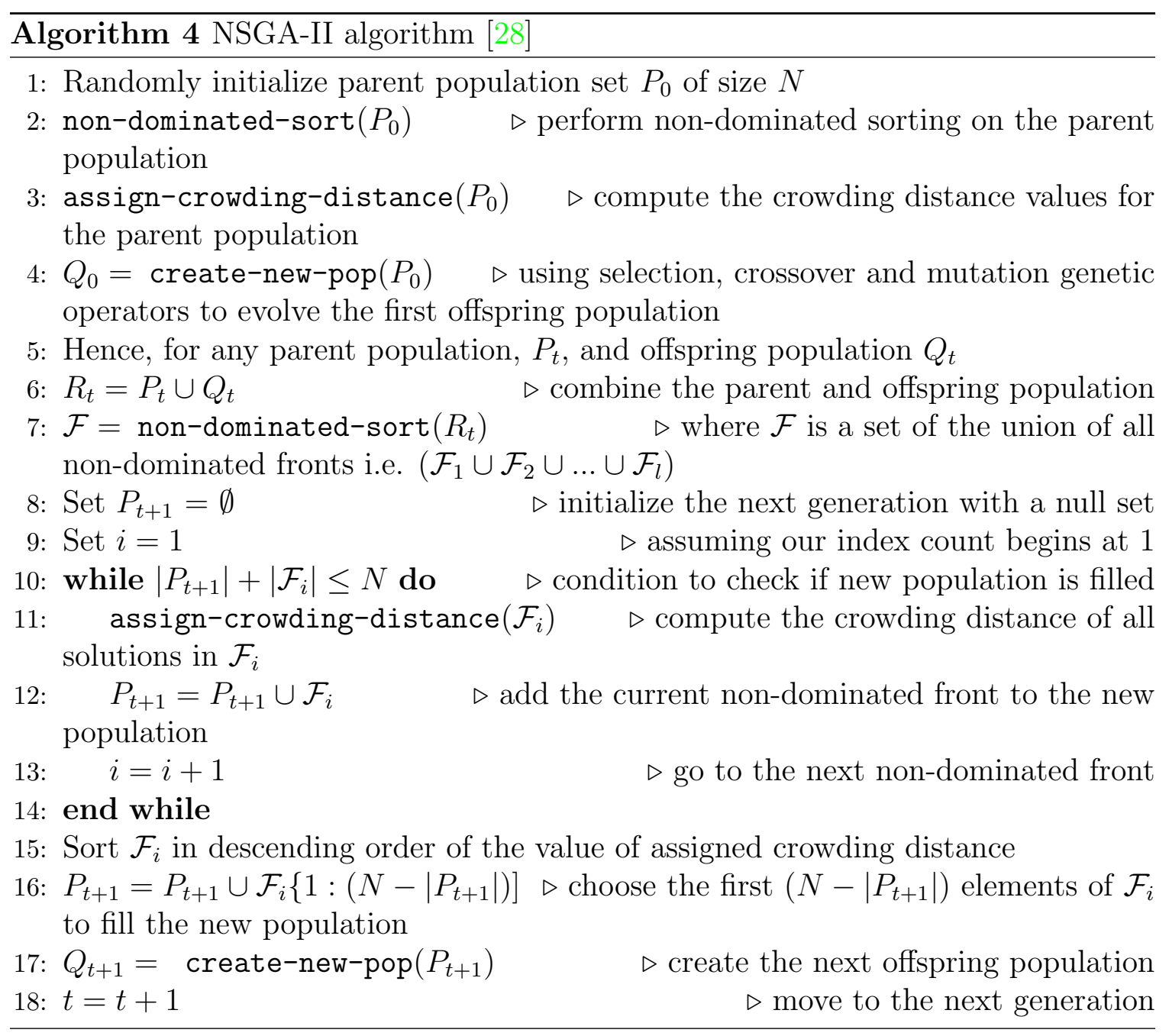




\subsubsection{Reference point-based non-dominated sorting algo- rithm (NSGA-III)}

The NSGA-III algorithm also introduced by Deb et al [29] is an extension of the original NSGA-II algorithm. The NSGA-III procedure follows the same framework outlined in the proposed NSGA-II algorithm with a slight modification to the selection operator. Unlike in the NSGA-II algorithm where maintaining diversity of the population members was accomplished by using crowding-distance operator, here, diversity is maintained by supplying and adaptively updating a number of well-spread reference points [29]. This, according to the authors, should result in the optimal points being more evenly distributed across the pareto-optimal front.

\subsubsection{Non-dominated sorting}

Let us consider an iteration of the NSGA-III algorithm for the multi-objective example problem stated in equation 3.2. Assume we have our initial parent population $P_{t}$ of size $N=5$, populated with solutions as shown in equation 3.26. On applying the genetic operators to the arbitrary parent population $P_{t}$, we create an offspring population $Q_{t}$ also of size $N=5$ also shown in 3.26. We then combine both the parent and offspring populations to give $R_{t}=P_{t} \cup Q_{t}$ of size $N=10$ as shown in equation 3.26 .

$$
\begin{aligned}
& P_{t}=\{9.25, \quad 0.70, \quad 9.28, \quad-7.69, \quad-8.97\} \\
& Q_{t}=\{-3.91, \quad 1.60, \quad 0.62, \quad 8.02, \quad 0.81\}
\end{aligned}
$$

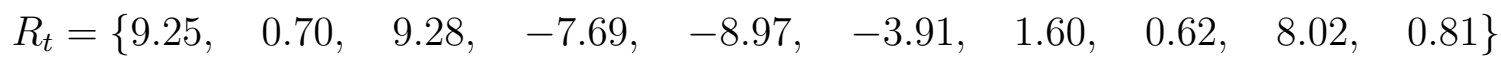


After performing the non-dominated sorting operation described in step 3.3.1.2 of the NSGA-II algorithm, the solutions are sorted into the non-dominated fronts shown in equation 3.27:

$$
\begin{aligned}
& \mathcal{F}_{1}=\{2, \quad 7, \quad 8, \quad 10\} \\
& \mathcal{F}_{2}=\{6\} \\
& \mathcal{F}_{3}=\left\{\begin{array}{ll}
4, & 9
\end{array}\right\} \\
& \mathcal{F}_{4}=\{1, \quad 5\} \\
& \mathcal{F}_{5}=\{3\}
\end{aligned}
$$

where $\mathcal{F}_{i}$ is the $i^{t h}$ non-dominated front. For example, the first non-dominated front contains Solutions 2, 7, 8 and 10, exactly the same as the NSGA-II algorithm.

\subsubsection{Determination of reference points}

To maintain a good spread of solutions among each generation's solution set, the NSGA-II algorithm employed a diversity preservation operation where crowdingdistance values are computed for each solution in the set as discussed in section 3.3.1.3. The crowding-distance value was computed by evaluating the absolute normalized difference summed across every objective for each solution. The crowding-distance values are then used in the algorithm's selection operation to choose solutions with the largest crowding-distance values within the same non-dominated front to populate the next parent population set.

The NSGA-III algorithm on the other hand, uses a set of predefined or usersupplied reference points to maintain diversity of the solutions which, according to the authors, should result in a uniform spread of pareto points across the objective space. This is an advantage over the crowding-distance operation which only ensured 
diversity-preservation in the selection operation of the NSGA-II algorithm. In this thesis, we focus only on employing a set of predefined reference points to preserve diversity.

The first step is to determine the position of the reference points on the objective space. These reference points can either be a predefined structured distribution or user supplied. A suitable structured distribution approach employed in this thesis was proposed by Das et al in [36] which places equally spaced reference points on a hyperplane, equally inclined to all objective axes with a unit intercept on each axis. On a $\mathbb{R}^{n}$ dimensional objective space, a hyper plane is an $(n-1)$-dimensional subspace. For example, on a 2-dimensional space (i.e. $\mathbb{R}^{2}$ ), a hyperplane is a line; while on a 3 -dimensional space (i.e. $\mathbb{R}^{3}$ ) a hyperplane is 2-dimensional plane.

According to Das et al in [36], we define a vector of equally spaced reference points as $\beta_{n}$ where $n$ represents the number of objectives and define $\delta(\delta>0)$ as the uniform spacing between two consecutive $\beta$ values. In other words, $\delta$ represents a step-size parameter. Also, if we assume $\frac{1}{\delta}$ is an integer for simplicity, the possible values that can be assumed by $\beta_{1}$ are:

$$
[0, \quad \delta, \quad 2 \delta, \ldots, 1]
$$

Now that we have values for $\beta_{1}$, let's define $m_{1}=\frac{\beta_{1}}{\delta}$, i.e., $\beta_{1}=m_{1} \delta$, hence, all corresponding $\beta_{i}$ elements must add up to one. Thus, the possible values of $\beta_{2}$ corresponding to values of $\beta_{1}$ are:

$$
\left[0, \quad \delta, \quad 2 \delta, \ldots, k_{2} \delta\right]
$$

where $k_{2}=\frac{1-\beta_{1}}{\delta}=\frac{1-m_{1} \delta}{\delta}$. Likewise, if we define $m_{2}=\frac{\beta_{1}}{\delta}$, the possible values of $\beta_{3}$ 
corresponding to values of $\beta_{1}=m_{1} \delta$ and $\beta_{2}=m_{2} \delta$ are:

$$
\left[0, \quad \delta, \quad 2 \delta, \ldots, k_{3} \delta\right]
$$

where $k_{3}=\frac{1-\beta_{1}-\beta_{2}}{\delta}=\frac{1-m_{1} \delta-m_{2} \delta}{\delta}$. Consequently, if $\beta_{i}=m_{i} \delta$, for $i=1, \ldots, j-1$, the possible values for $\beta_{j}$ are:

$$
\left[0, \quad \delta, \quad 2 \delta, \ldots, k_{j} \delta\right]
$$

where $k_{i}=\frac{1-\sum_{i=1}^{j-1} m_{i}}{\delta}$. The last $\beta$ can be defined as:

$$
\beta_{n}=1-\sum_{i=1}^{n-1} \beta_{i}
$$

A simpler way of looking at this approach is to think of it as a tree diagram where each node along the branches represents a $\beta_{i}$ vector and each path from the root node to the leaf node represents a reference point coordinate on the normalized objective space. For example, if we define $\delta$ as 0.25 (i.e. we want $\frac{1}{0.25}=4$ divisions along each objective) in a three-objective problem, the values of the reference points $\left(\beta_{3}\right)$ are shown in the tree diagram of figure 3.9.

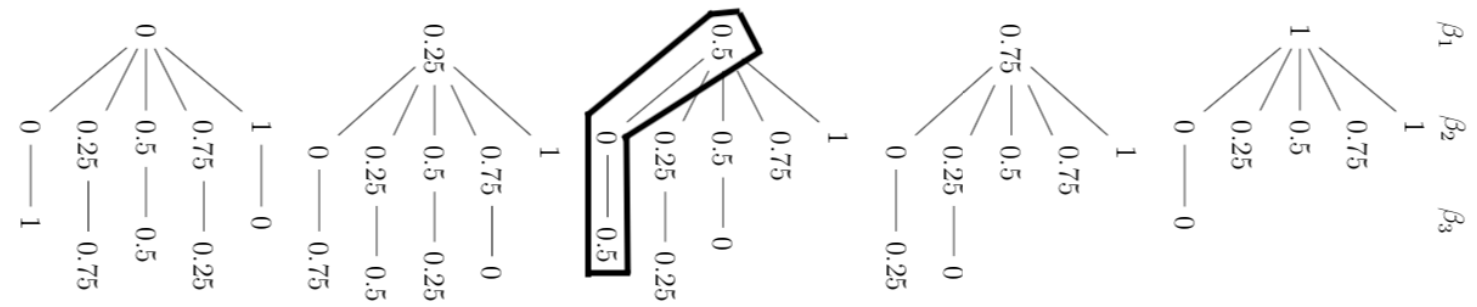

Figure 3.9: Tree diagram for generating reference points for a 3-objective problem with $\delta=0.25$

The coordinates of the reference points on the objective space are made up of the values on the nodes along each branch (i.e. $\left.\left(\beta_{1}, \beta_{2}, \beta_{3}\right)\right)$ as shown in figure 3.9. 
Note that the branches that do not continue to the third $\left(\beta_{3}\right)$ level are not considered, this is because the elements of the reference point vector must add up to one. The 15 reference points form a triangle on the objective space with the vertices at $(1,0,0),(0,1,0)$ and $(0,0,1)$ as shown in figure 3.10 .

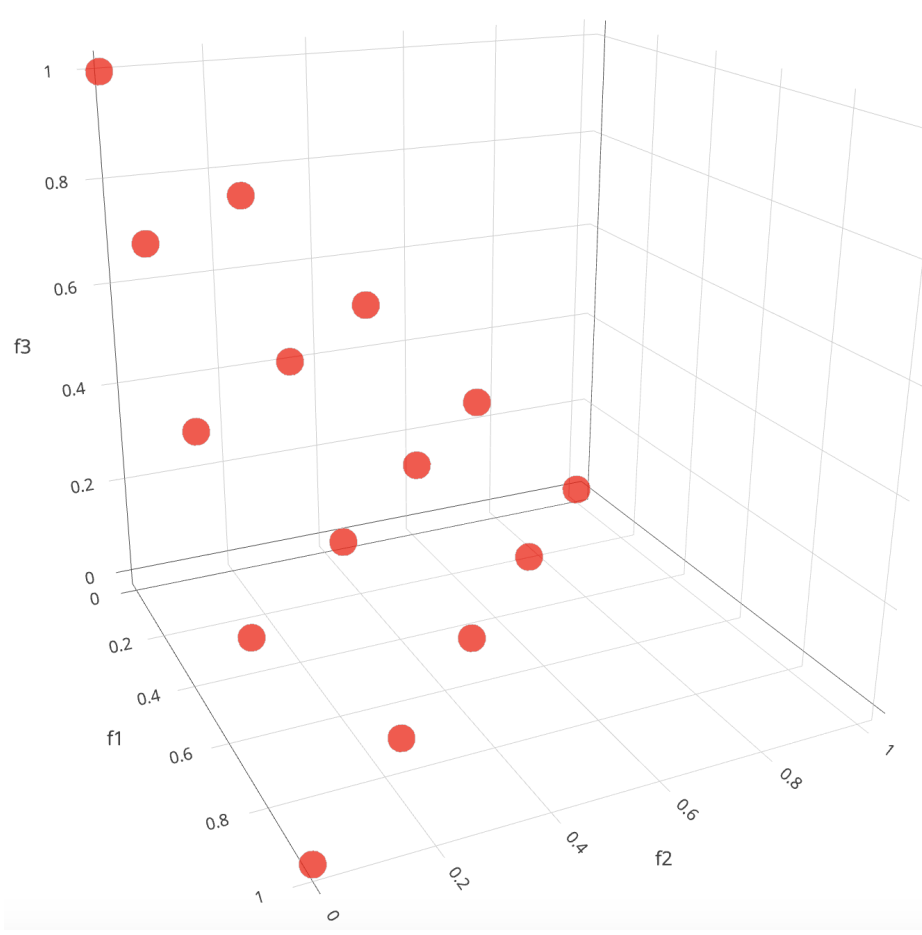

Figure 3.10: Reference point coordinates on a normalized hyper plane for a 3objective problem with $\delta=0.25$

A quick way to compute the number of reference points, $\mathrm{H}$, is to use the formula in equation 3.33; where $M$ is the number of objectives and $p$ is the number of divisions along each objective. For example, if $M=3$ and $p=4$ : hence, $H=\left(\begin{array}{c}3+4-1 \\ 4\end{array}\right)$ and applying the combination formula, $H={ }^{6} C_{4}=15$ reference points as shown in figure 3.10 .

$$
H=\left(\begin{array}{c}
M+p-1 \\
p
\end{array}\right)
$$


A rule of thumb proposed by the authors in [29] is to choose the number of divisions, $p$ in such a way that the number of reference points, $H$ is approximately equal to the population size, $N$ (i.e. $H \approx N$ ). Therefore, to determine the reference points for our example problem, we set the number of divisions $p=9$, hence, the number of reference points, $H=\left(\begin{array}{c}2+9-1 \\ 9\end{array}\right)=10$ reference points. The step-size parameter, $\delta=\frac{1}{9}=0.11$. Since we have two objectives (i.e. $M=2$ ), we will have two $\beta$ levels (i.e. we will stop at $\beta_{2}$ ). Therefore, the possible values for the $\beta_{1}$ vector are computed as shown in equation 3.28. Hence, the first element $\beta_{1}^{1}=0$, the second $\beta_{1}^{2}=2 \delta=0.22$, the third $\beta_{1}^{3}=3 \delta=0.33$ and finally the last element $\beta_{1}^{10}=1$. Figure 3.11 shows the possible values that can be assumed by $\beta_{1}$. The next $\beta$ level (i.e.

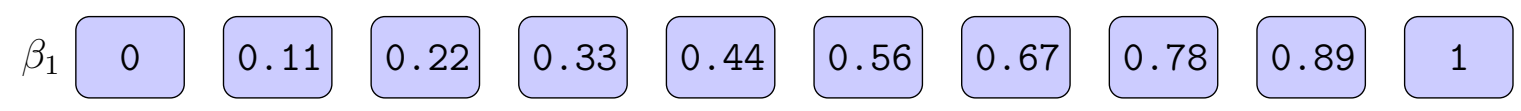

Figure 3.11: Possible values for $\beta_{1}$

$\left.\beta_{2}\right)$ is the last because our example problem only has two objectives, hence, we will apply the formula in equation 3.32 to compute the corresponding $\beta_{2}$ element for each value in $\beta_{1}$. Therefore, for the first element $\beta_{2}^{1}=1-\beta_{1}^{1}=1-0=1$; for the second $\beta_{2}^{2}=1-\beta_{1}^{2}=1-0.11=0.89$ and for the last element $\beta_{2}^{10}=1-\beta_{1}^{10}=1-1=0$.

Figure 3.12 , shows the possible values for $\beta_{2}$ corresponding to the elements in $\beta_{1}$.

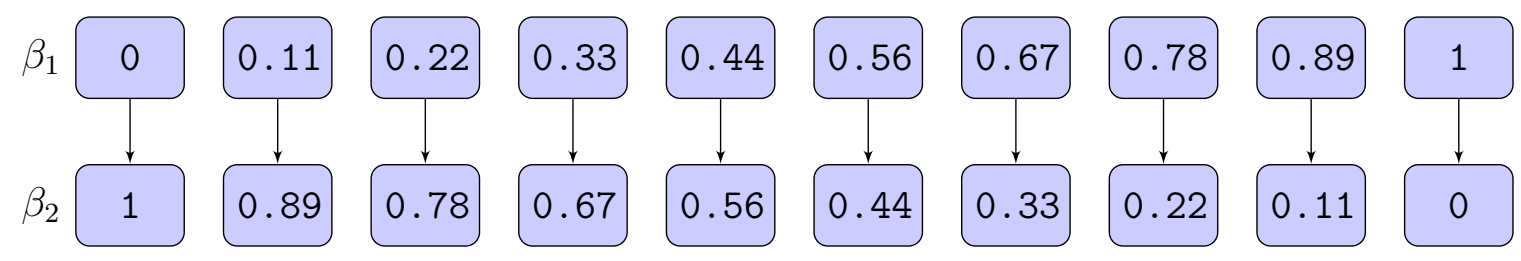

Figure 3.12: Possible values for $\beta_{2}$

The reference points on our example problem's normalized objective space are 
extracted from the values on the $\beta$ node levels in figure 3.12 and expressed in the form $\left(\beta_{1}, \beta_{2}\right)$. Hence, the 10 reference points are:

$$
\begin{aligned}
& \operatorname{Ref}=\{(0,1),(0.11,0.89),(0.22,0.78),(0.33,0.67),(0.44,0.56), \\
& (0.56,0.44),(0.67,0.33),(0.78,0.22),(0.89,0.11),(1,0)\}
\end{aligned}
$$

Figure 3.13 , shows the plot of the reference points on a normalized objective space.

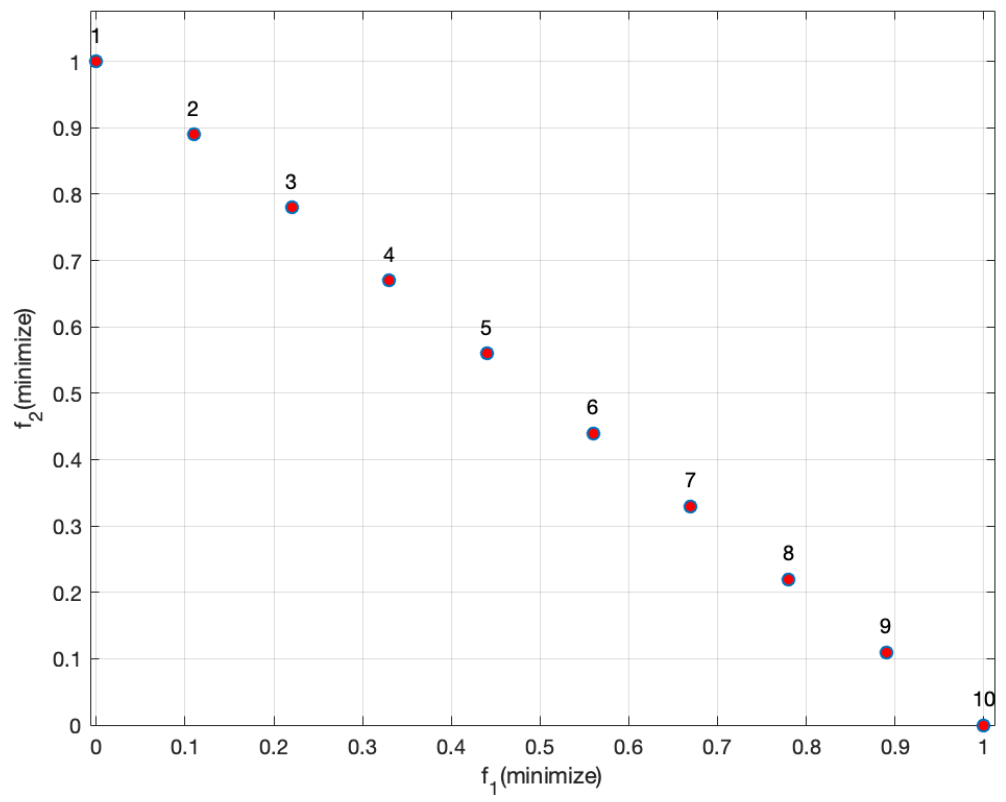

Figure 3.13: Plot of the reference points on the normalized space

\subsubsection{Adaptive normalization of population members}

After determining the reference points, the next procedure is to adaptively normalize the objective function values of the population members. The first thing to do is to determine the ideal (or optimal) point of the combined population, $R_{t}$. The algorithm assumes that all objectives are to be minimized, therefore if there is an objective that 
needs to be maximized, we simply need to multiple the function by -1 . Hence, we determine the ideal point along each objective by identifying the minimum values, $z_{i}^{\min }$ for $i=1,2, \ldots, M$ (where $M$ is the number of objectives) for each objective function. Evaluating the solutions in equation 3.26 where $f_{1}=x^{2}$ and $f_{2}=(x-2)^{2}$, we have:

$f_{1}(x)=\{85.56, \quad 0.49, \quad 86.12, \quad 59.14, \quad 80.46, \quad 15.29, \quad 2.56, \quad 0.38, \quad 64.32, \quad 0.66\}$ $f_{2}(x)=\{52.56, \quad 1.69, \quad 53.00, \quad 93.90, \quad 120.34, \quad 34.93, \quad 0.16, \quad 1.90, \quad 36.24, \quad 1.42\}$

We can see from 3.35 that the minimum value of the first objective (i.e. $z_{1}^{\min }$ ) is the $8^{\text {th }}$ member in $f_{1}$ (i.e. $f_{1}^{8}=0.38$ ) while that of the second objective, (i.e. $z_{2}^{\text {min }}$ ) is the $7^{\text {th }}$ member in $f_{2}$ (i.e. $\left.f_{2}^{7}=0.16\right)$. Therefore the ideal point, $\bar{z}=\left(z_{1}^{\min }, z_{2}^{\min }, \ldots, z_{M}^{\min }\right)$ for combined population, $R_{t}$ in equation 3.26 is given as:

$$
\bar{z}=(0.38, \quad 0.16)
$$

The next step is to translate each value for both objectives by subtracting from each objective value $f_{i}$ the corresponding objective's minimum value, $z_{i}^{\text {min }}$. Thus, computing the translated values: $f_{i}^{\prime}=f_{i}(x)-z_{i}^{\text {min }}$ for each objective, we have:

$$
\begin{aligned}
& f_{1}^{\prime}(x)=\{85.18, \quad 0.11, \quad 85.73, \quad 58.75, \quad 80.08, \quad 14.90, \quad 2.18, \quad 0, \quad 63.94, \quad 0.27\} \\
& f_{2}^{\prime}(x)=\left\{\begin{array}{llllllllll}
52.40, & 1.53 & 52.84 & 93.74 & 120.18 & 34.77 & 0 & 1.74 & 36.08 & 1.26
\end{array}\right\}
\end{aligned}
$$

where, $f_{1}^{\prime}$ and $f_{2}^{\prime}$ are the translated versions of objectives $f_{1}$ and $f_{2}$ in equation 3.35 respectively. We can see from equation 3.37, that translating the objectives makes 
the ideal point along each objective zero.

The next step is to identify the extreme points, $z_{i}^{\max }$ along each objective. This is done by finding the solution $\left(x \in R_{t}\right)$ that makes the following scalarizing function in equation 3.38 minimum:

$$
S F(\mathbf{x}, \mathbf{w})=\max _{i=1}^{M} \frac{f_{i}^{\prime}(x)}{w_{i}}, \quad \text { for } x \in R_{t}
$$

where, $\mathbf{x}$ is a vector of the values of both objectives of the same index (i.e. $\mathbf{x}=\left[x_{i}^{1}, x_{i}^{2}\right]$ ) and the weight vector $\mathbf{w}$ is a vector of the axis direction for both objectives (i.e. $\mathbf{w}=\left[w_{i}^{1}, w_{i}^{2}\right]$. All the weight values are set to a very small number (e.g. $\epsilon=10^{-6}$ ) except the weight vector of the current objective, $i$, will be set to 1 (i.e. $w_{i}^{i}=1$ ).

Therefore, to compute the extreme point for the first objective in our example problem, $f_{i}^{\prime}(\mathbf{x})=\left[f_{1 i}^{\prime}(x), f_{2 i}^{\prime}(x)\right]$ for $i=1,2, \ldots, 10$; where $f_{1 i}^{\prime}(x)$ and $f_{2 i}^{\prime}(x)$ are the minimum values of the first and second objectives in equation 3.37 respectively. Since this is the first objective, the weight value $w_{i}^{1}=1$; hence the weight vector will be $\mathbf{w}=\left[1, \epsilon=10^{-6}\right]$.

$$
\begin{aligned}
& i=1 ; \\
& \quad S F_{1}^{1}=\max \left[\frac{85.18}{1}, \frac{52.40}{10^{-6}}\right]=52400000 \\
& i=2 ; \\
& \quad S F_{1}^{2}=\max \left[\frac{0.11}{1}, \frac{1.53}{10^{-6}}\right]=1530000 \\
& \quad \vdots \quad S F_{1}^{10}=\max \left[\frac{0.27}{1}, \frac{1.26}{10^{-6}}\right]=1260000
\end{aligned}
$$


Equation 3.39 shows how the scalarizing function in equation 3.38 along the first objective was computed using the translated values in equation 3.37. Now we identify the solution that makes the scalarizing function along the first objective minimum.

$$
\begin{aligned}
& S F_{1}=\{52400000, \quad 1530000, \quad 52840000, \quad 93740000, \quad 120180000, \\
& 34770000, \quad 0, \quad 1740000, \quad 36080000, \quad 1260000\}
\end{aligned}
$$

Hence, looking at equation 3.40, the smallest value in set $S F_{1}$ is 0 which corresponds to Solution 7. Thus, the maximum value along the first objective is obtained by extracting (from equation 3.37) the translated values (in both objectives) of the solution that makes the scalarized function minimum as shown below:

$$
z_{1}^{\max }=(2.18,0)
$$

where 2.18 and 0 are the translated values along the first and second objectives in equation 3.37 respectively; hence the point 2.18 is the intercept, $a_{1}$ along the $f_{1}$ objective. Following the same steps equation 3.42 shows how we compute the extreme point along the second objective, we set $w_{i}^{2}=1$, hence, the weight vector 
$\mathbf{w}=\left[\epsilon=10^{-6}, 1\right]$.

$$
\begin{gathered}
i=1 ; \\
S F_{2}^{1}=\max \left[\frac{85.18}{10^{-6}}, \frac{52.40}{1}\right]=85160000 \\
i=2 ; \\
S F_{2}^{2}=\max \left[\frac{0.11}{10^{-6}}, \frac{1.53}{1}\right]=110000 \\
\vdots \\
i=10 ; \\
S F_{2}^{10}=\max \left[\frac{0.27}{10^{-6}}, \frac{1.26}{1}\right]=270000 \\
S F_{2}=\{85160000, \quad 110000, \quad 85730000, \quad 58750000, \quad 80080000, \\
14900000, \quad 21800000, \quad 0, \quad 63940000, \quad 270000\}
\end{gathered}
$$

Equation 3.43 shows the scalarizing function set, $S F_{2}$ along the second objective. We can see from the set that Solution 8 gives the smallest value in the set. Hence, the maximum value along the second objective corresponds to the translated objective values (in equation 3.37) for Solution 8 as shown in equation 3.44:

$$
z_{2}^{\max }=(0,1.74)
$$

hence 1.74 is the intercept, $a_{2}$ along the $f_{2}$ axis. Finally, the two objective functions can be normalized using the following formula:

$$
f_{i}^{\prime \prime}(\mathbf{x})=\frac{f_{i}^{\prime}(\mathbf{x})}{a_{i}}, \quad \text { for } i=1,2, \ldots, M
$$

where $f_{i}^{\prime}$ are the translated objective values in equation 3.37 and $a_{i}$ is the axis intercept 
(i.e. $a_{1}=2.18$ and $a_{2}=1.74$ ). Therefore, we normalize the first objective by dividing every element of the translated objective in equation 3.37 by 2.18 and the second objective by dividing by 1.78 to give:

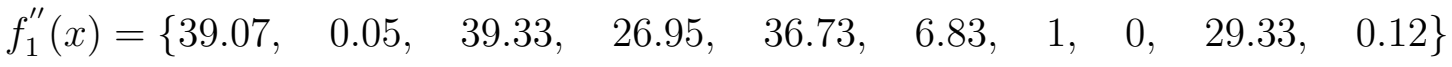

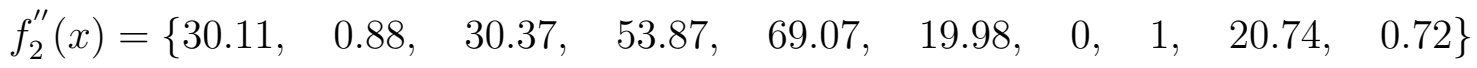

Figure 3.14 shows a plot of the normalized objectives. We can see from equation 3.46 that the intercepts on each normalized axis is now located at $f_{i}^{\prime \prime}=1$ and the hyperplane we construct with the reference points and the intercept points will make $\sum_{i=1}^{M}=f_{i}^{\prime \prime}=1$ as shown in figure 3.15 below.

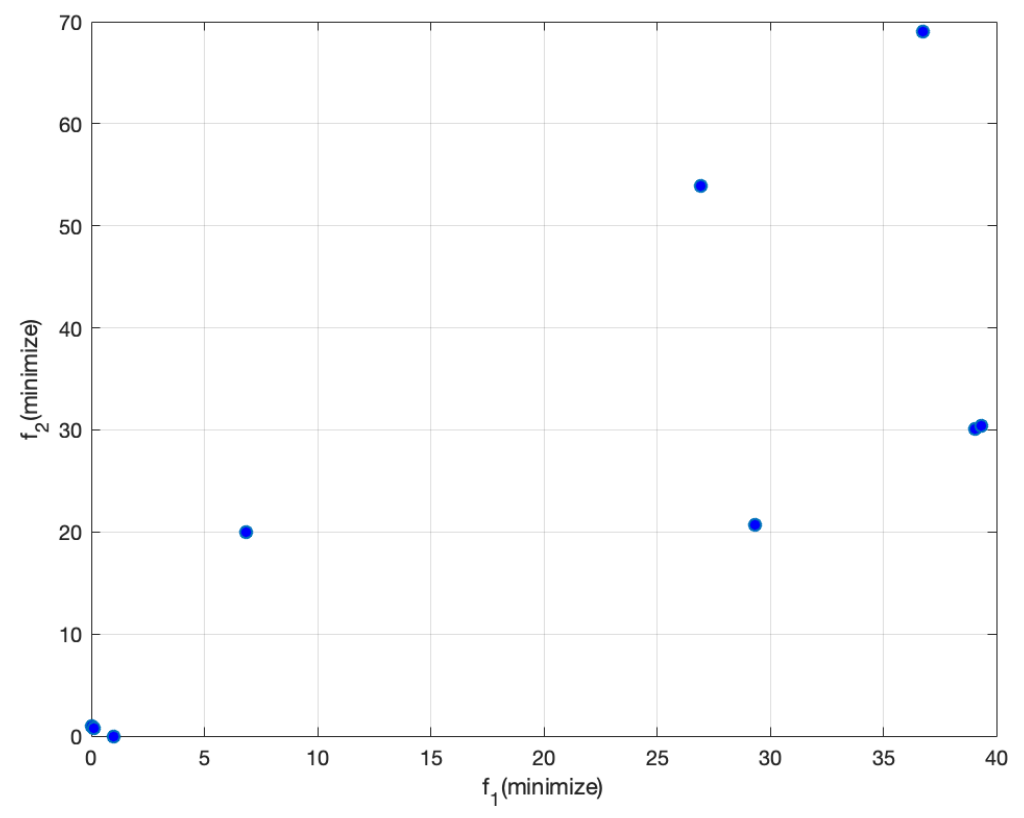

Figure 3.14: Plot of the normalized space showing the normalized objective points 


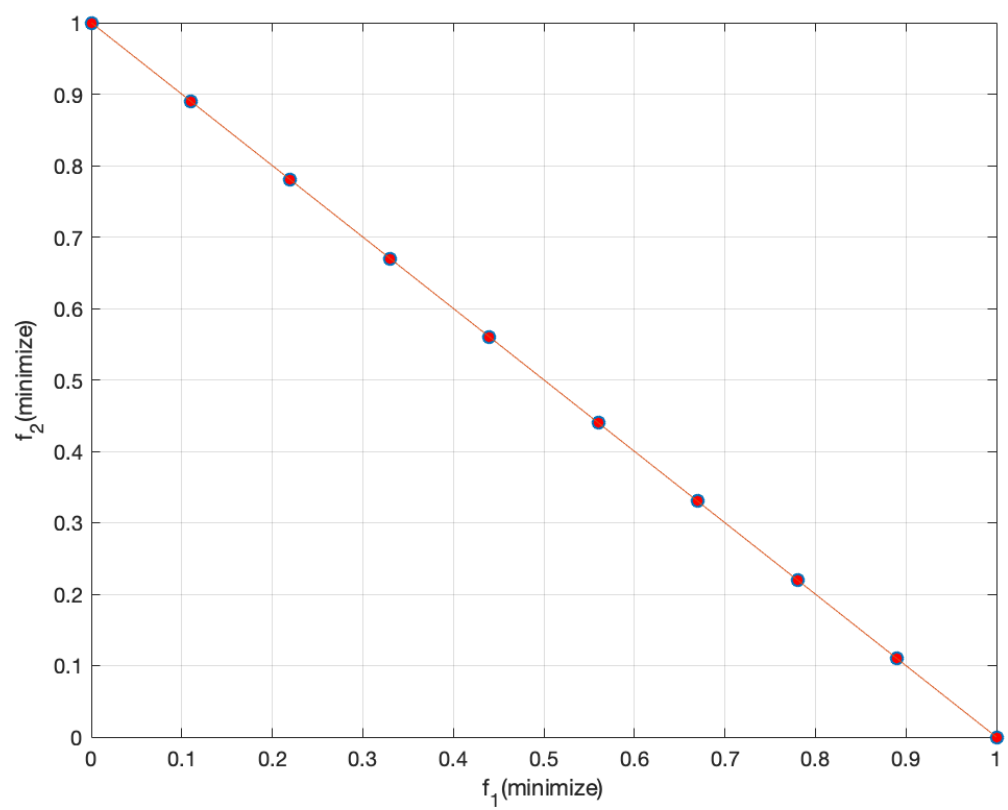

Figure 3.15: Plot of the normalized space showing the hyperplane and the reference points

\subsubsection{Association operation}

After adaptively normalizing each objective, we need to associate each population member in the population with a reference point in the normalized objective space. To do this, we first need to define a reference line corresponding to each reference point which projects from the origin through each reference point. We then compute the perpendicular distance from each population member in the normalized population to each reference line as shown in figure 3.16 using the arbitrary 3-objective problem introduced earlier. The reference point whose reference line is closest to a population member in the normalized objective space is considered to be associated with that population member.

Applying the association operation to our example problem in equation 3.2, we first define a reference line corresponding to each reference point in equation 3.34 


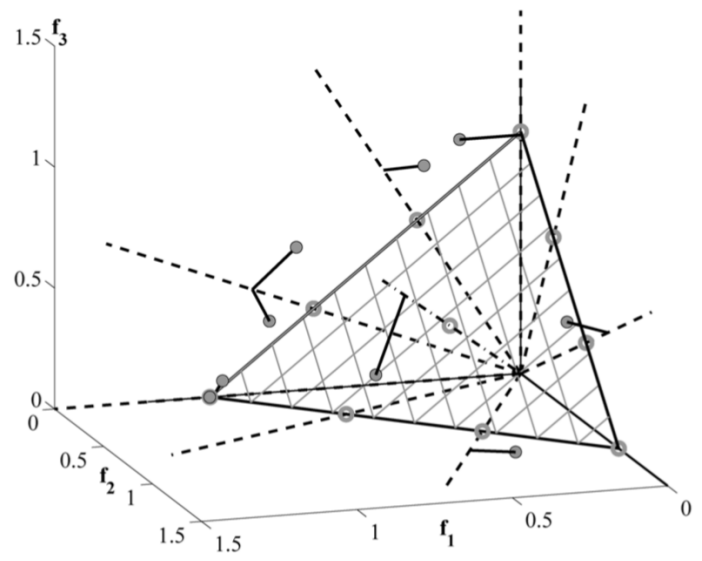

Figure 3.16: Illustration of how population members are associated with reference lines [29]

which joins the reference point to the origin. To make computations easy, we define the lines using the standard form of the equation of a line (i.e. $A x+B y+C=0$ ).

$$
\begin{aligned}
& y=m x+b ; \text { Let } m=\frac{A}{B} \\
& \text { Hence: }-m x+y-b=0 \\
& -\frac{A}{B} x+y-b=0 \\
& -A x+B y-B b=0 \quad \text { where y-intercept: } C=-B b
\end{aligned}
$$

Therefore, we convert the slope-intercept form (i.e. $y=m x+b$, where $m$ is the slope of the line and $b$ is the y-intercept), into the standard form as shown in equation 3.47. Equation 3.48 details how the reference lines are defined and shown graphically in 
figure 3.17 .

$$
\begin{aligned}
& \text { For origin }=(0,0) \text { and } \operatorname{Ref}(1)=(0,1) \\
& m=\frac{1-0}{0-0}=\frac{1}{0}=\frac{A}{B} \text { in equation } 3.47 \\
& \text { y-intercept }: b=1 \\
& \text { Reference line } 1: \quad x=0 \\
& \text { For origin }=(0,0) \text { and } \operatorname{Ref}(2)=(0.11,0.89) \\
& m=\frac{0.89-0}{0.11-0}=\frac{0.89}{0.11}=\frac{A}{B} \\
& \text { y-intercept }: b=0 \\
& \text { Reference } l \text { ine } 2: \quad 0.89 x+0.11 y=0 \\
& \vdots \\
& \text { For origin }=(0,0) \text { and } \operatorname{Ref}(10)=(1,0) \\
& m=\frac{0-0}{1-0}=\frac{0}{1}=\frac{A}{B} \\
& \text { y-intercept }: b=0 \\
& \text { Reference } l i n e 10: \quad y=0
\end{aligned}
$$

To associate the normalized objective space population members in equation 3.46, we take each pair of the normalized population member objective evaluations $\left(f_{1}^{\prime \prime}(x), f_{2}^{\prime \prime}(x)\right)$ in equation 3.46 , compute its perpendicular distance to each reference line defined in equation 3.48 using the formula:

$$
\frac{\left|A f_{1}^{\prime \prime}(x)+B f_{2}^{\prime \prime}(x)+C\right|}{\sqrt{A^{2}+B^{2}}}
$$

where the coefficients $A, B$ and $C$ were evaluated in equation 3.48. We then associate 


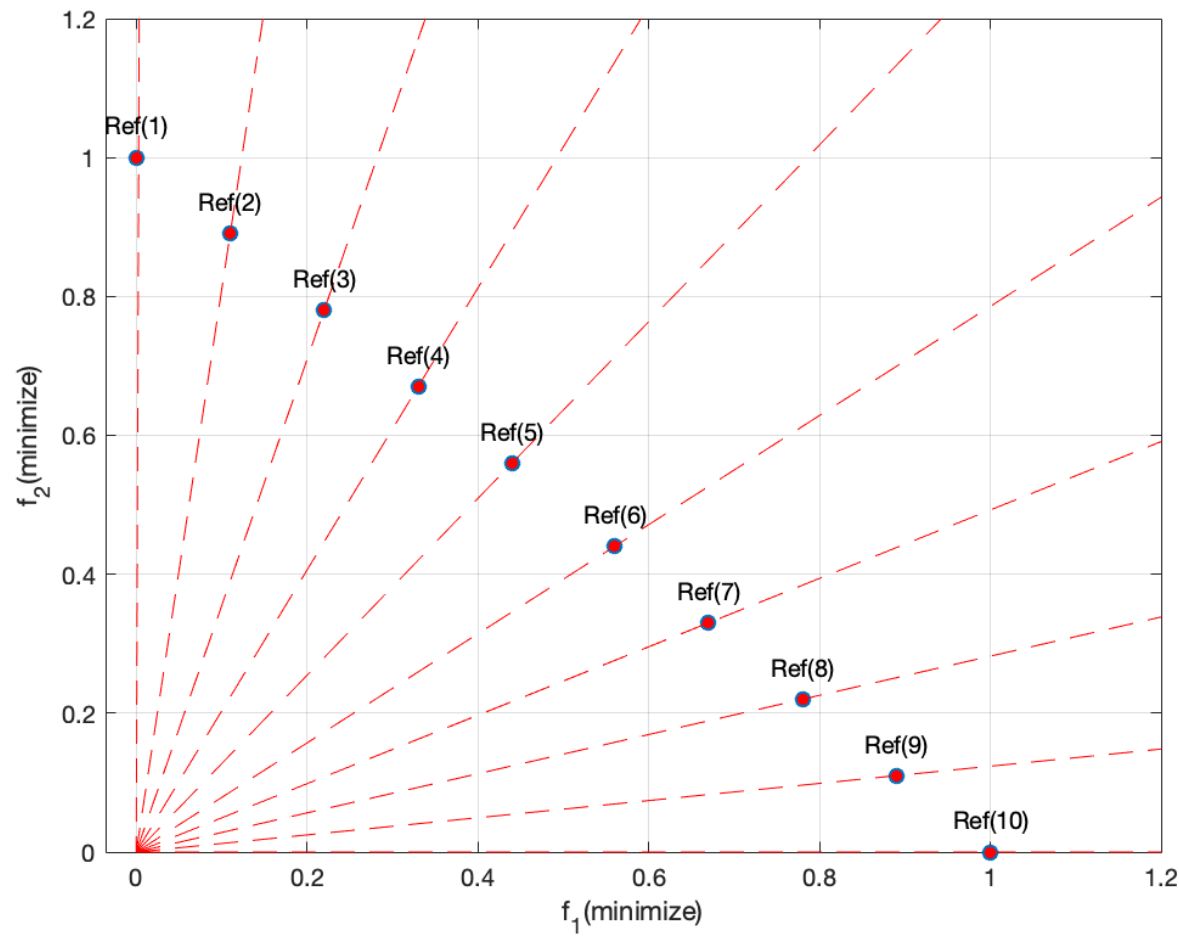

Figure 3.17: Plot of the reference lines 
each normalized population member to the reference point whose reference line is closest to it.

$$
\begin{aligned}
& \text { For the } 1^{\text {st }} \text { normalized solution: }(39.07,30.11) \\
& 1^{\text {st }} \text { reference line: } \operatorname{Ref}(1): \quad x=0 \\
& \text { dist }(1)=f_{1}^{\prime \prime}(x)=39.07 \\
& 2^{\text {nd }} \text { reference line: } \operatorname{Ref}(2): 0.89 x+0.11 y=0 \\
& \text { dist (2) }=\frac{0.89(39.07)+0.11(30.11)}{\sqrt{0.89^{2}+0.11^{2}}}=34.87 \\
& \vdots \\
& 10^{t h} \text { reference line: } \operatorname{Ref}(10): \quad y=0 \\
& \text { dist (10) }=f_{2}^{\prime \prime}(x)=30.11
\end{aligned}
$$

Equation 3.50 illustrates how the distance from the first normalized solution to each reference line is computed. Equation 3.51 shows a set of the distance from the first normalized solution (i.e. $(39.07,30.11)$ in equation 3.46$)$ to each reference line.

dist $=\{39.07, \quad 34.87, \quad 28.79, \quad 20.85, \quad 11.45, \quad 0.44, \quad 9.35, \quad 17.97, \quad 24.94, \quad 30.11\}$

We can see from equation 3.51 that the smallest distance is 0.44 which corresponds to the $6^{\text {th }}$ reference line. In the same way we compute for the associated reference lines for the other normalized population members.

$$
\text { assRefLines }=\{6,1,6,4,4,3,10,1,6,2\}
$$

Equation 3.52 shows the set of the reference lines each normalized solution is associated with. For example, solution 2 is associated with reference line 1, solution 10 is 
associated with reference line 2 and solutions 7 and 8 are associated with reference lines 10 and 1 respectively as shown in figure 3.18.

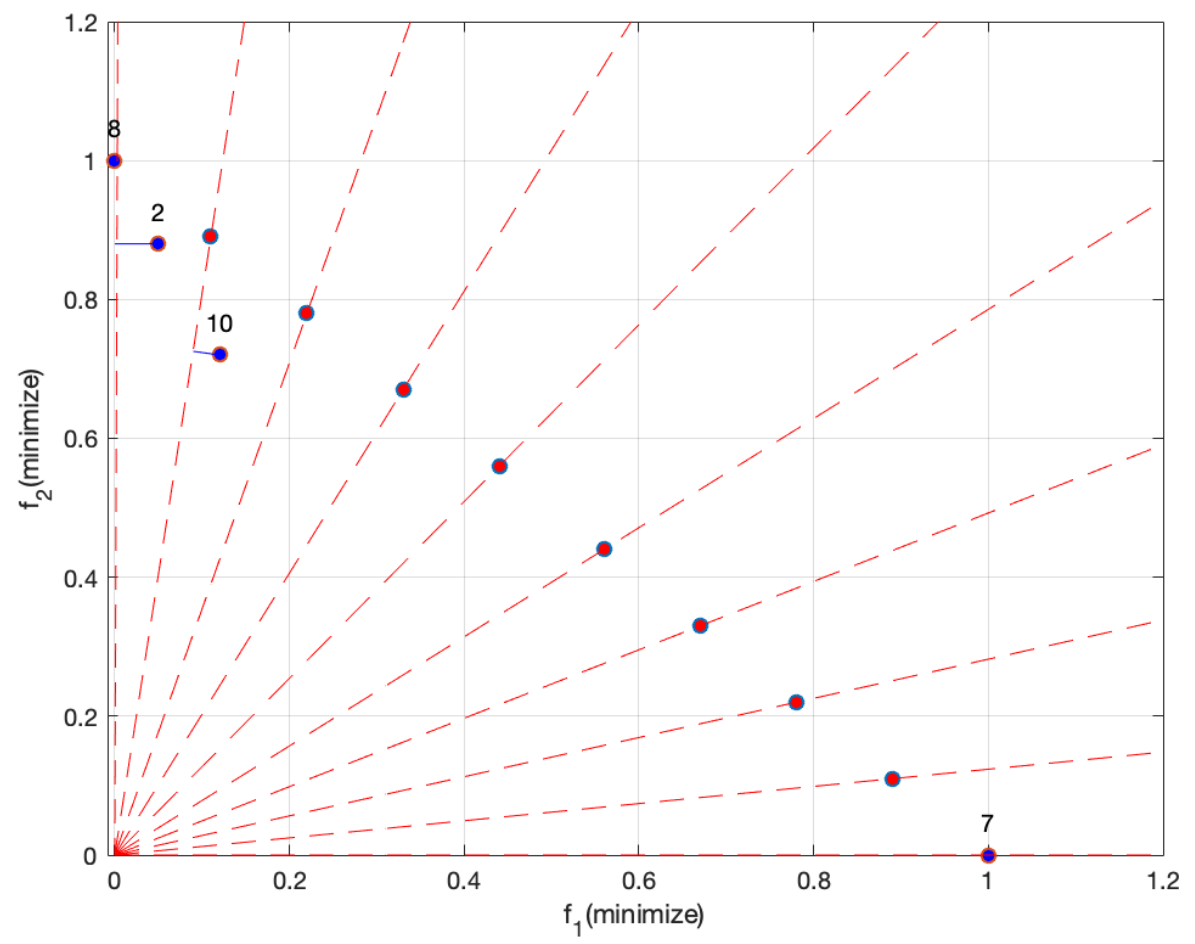

Figure 3.18: Plot showing normalized solutions associated to reference lines

\subsubsection{Diversity-preservation operation}

The next step is to evolve the next parent population, $P_{t+1}$ as described in section 3.3.1.5 of the NSGA-II algorithm. Now we depart from the NSGA-II algorithm. To preserve diversity $\mathrm{n}$ the process of evolving the next parent population in the NSGAII algorithm, we would have employed the crowded-distance operation as described in section 3.3.1.5. This time in the NSGA-III algorithm, things are done a bit differently in the sense that diversity in the population is maintained by employing the reference point-based association operation. 
To better illustrate how the diversity-preservation operation works, we will not pick a population of 5 for the next generation, $P_{t+1}$, instead we will choose a population of 6 (i.e. $\left|P_{t+1}\right|=6$ ). This is because choosing 5 solutions will mean copying all the solutions in the first and second non-dominated fronts in equation 3.27 and the diversity-preservation operation would not be employed. Therefore, all the solutions in the first and second non-dominated fronts of equation 3.27 will make it into the next parent population $P_{t+1}$ as shown in equation 3.53.

$$
\mathcal{F}_{1} \cup \mathcal{F}_{2}=\{2, \quad 7, \quad 8, \quad 10, \quad 6\}
$$

To determine the solution out of the two in the last non-dominated front $\mathcal{F}_{l}=\mathcal{F}_{3}=$ $\left\{\begin{array}{l}4, \\ 9\end{array}\right\}$ in equation 3.27. We will apply the diversity-preservation operation proposed by the authors [29]. The diversity-preservation operation helps maintain diversity in the new parent population, $P_{t+1}$ in situations where multiple or no solutions in the new parent population are associated with a reference point, $j$. The steps in the diversity-preservation operation are enumerated below:

1. The first thing is to set-up a diversity-counting operator, $\rho_{j}$, for each reference point which defines how many solutions in the next population $\left(P_{t+1}\right)$ in equation 3.53 are associated with the same reference point as shown below:

$$
\rho=\left\{\begin{array}{llllllllll}
2, & 1, & 1, & 0, & 0, & 0, & 0, & 0 & 0, & 1
\end{array}\right\}
$$

where $\rho_{1}=2$ is the diversity-counting operator for reference point 1 and it has 2 solutions in the new population $P_{t+1}$ associated with it.

2. Next, we identify the reference point(s) with minimum $\rho_{j}$ and if multiple exist, we randomly select one. In our case, we have a 6 minimum reference points, 
namely: $\rho_{4}, \rho_{5}, \rho_{6}, \rho_{7}, \rho_{8}$ and $\rho_{9}$ with their values equal to 0 .

3. If chosen $\rho_{j}=0$, that is, no solution in the next population $P_{t+1}$ is associated with the reference point $j$, then we have two possible options:

a The last front, $\mathcal{F}_{l}$ does not have any member associated with the reference point. In this case, the reference point is discarded from further consideration for the current generation. Assume we randomly selected $\rho_{7}$ from the minimum reference points. Since reference point 7 in equation 3.54 does not have any member in third non-domination front, $\mathcal{F}_{3}$ in equation 3.27 associated with it, we would skip it and randomly pick another minimum reference point from the remaining 5 .

b There exists one or more members of front $\mathcal{F}_{l}$ associated with the reference point. In this case, the solution with the shortest perpendicular distance is added to the new generation $\left(P_{t+1}\right)$ and the corresponding reference point diversity-count is incremented by one. Assume we randomly selected $\rho_{4}$ from the minimum reference points. Since solution 4 in the third non-dominated front in equation 3.27 is associated with reference point $4, \rho_{4}=4$ in equation 3.52, we will add solution 4 to the new parent population and stop. Therefore, our next generation's population will be:

$$
P_{t+1}=\{2, \quad 7, \quad 8, \quad 10, \quad 6, \quad 4\}
$$

4. For $\rho_{j} \geq 1$, that is, at least one member of the next generation $\left(P_{t+1}\right)$ is associated with the reference point (e.g. $\rho_{1}, \rho_{2}, \rho_{3}$ and $\rho_{10}$ ), a randomly chosen solution from the population members for the last front $\left(\mathcal{F}_{l}\right)$ associated with the reference point is added to fill up the next generation population and the 
diversity-count of the reference point is incremented by one.

5. The diversity-preservation is repeated a total of $k$ times, where $k$ represents the remaining solutions needed to fill up the next population.

Figure 3.19 shows the pareto-optimal front for the solution to the example problem in equation 3.2, where the population size $N=100$ and the algorithm terminated after 100 iterations. In figure 3.19, the axes represent the two objective function values in the multi-objective problem in equation 3.2 and the points on the graph represent solutions in the final population. The NSGA-III algorithm is summarized in algorithm 5 .

At this point it is important to point out that a direct comparison between the pareto fronts of the algorithms from a single simulation run would not be a good basis to judge the performance of the algorithms since they are stochastic algorithms. Therefore, we compared the algorithms objectively in section 3.4.1. The algorithm has a computational complexity of $O\left(N^{2} \log ^{M-2} N\right)$ or $O\left(N^{2} M\right)$, whichever is higher; where $M$ is the number of objectives and $N$ is the population size. 


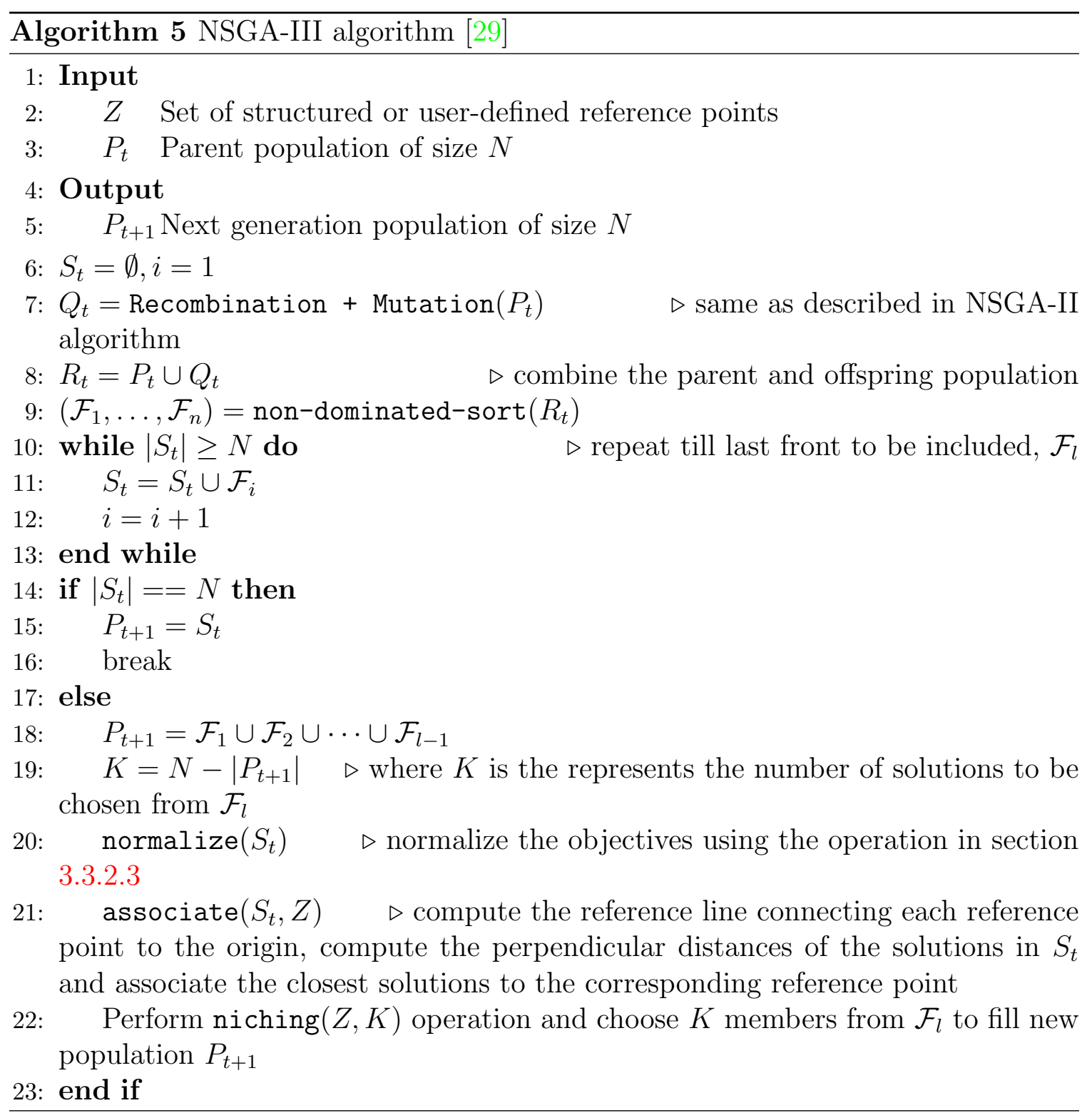




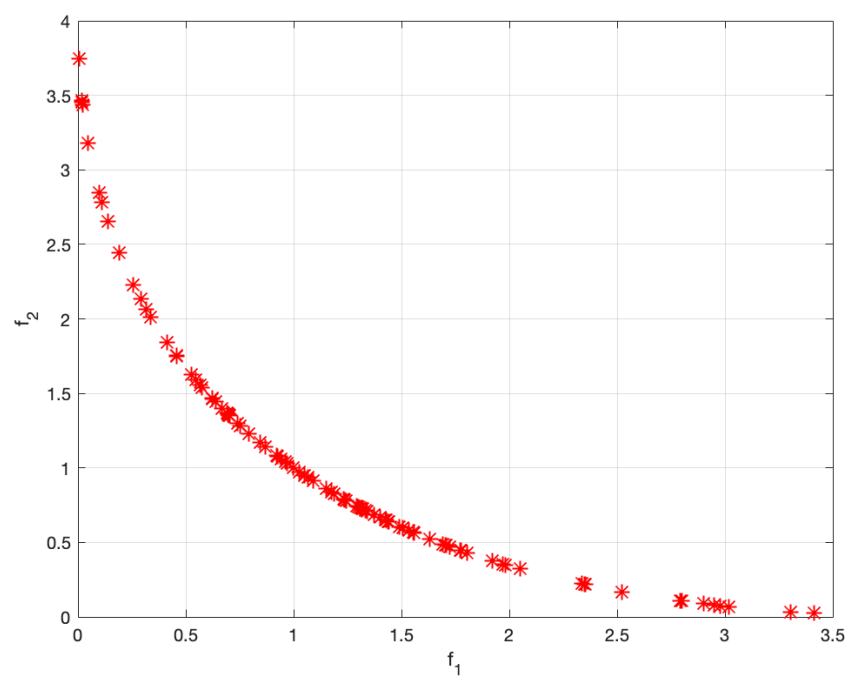

Figure 3.19: Pareto-optimal front showing solution to equation 3.2 using NSGA-III algorithm

\subsubsection{The improved Strength Pareto Evolutionary Algo- rithm (SPEA2)}

The improved strength pareto evolutionary algorithm (SPEA2) introduced by Zitzler et al in [31] is a technique for finding the pareto-optimal set of solutions in multiobjective optimization problems. The SPEA2 algorithm employs an external archive set to store the optimal solutions in each generation which corresponds to the highest ranked solutions in the NSGA-II and NSGA-III algorithms. The subsequent subsections below describe the steps with which the algorithm achieves this.

\subsubsection{Initialize parent population and archive set}

As with previously described evolutionary algorithms, we randomly initialize the parent population, $P_{0}$, of size $N$ and at the same time set up an empty set, $\bar{P}_{0}$ (of size $\left.\bar{N}\right)$, called the archive set, to maintain and update the highest ranked pareto-optimum solutions as the algorithm proceeds. Once again we will start with the same parent 
population as we did in the NSGA-II and NSGA-III algorithms, where we have the randomly initialized parent population shown in equation 3.56 for the multi-objective problem in equation 3.2; where $P_{0}$ is of size $N=10$ and the empty archive set, $\bar{P}_{0}$ is predefined with a maximum size of $\bar{N}=10$.

$$
\begin{aligned}
& P_{0}=\{9.25, \quad 0.70, \quad 9.28, \quad-7.69, \quad-8.97, \quad-3.91, \quad 1.60, \quad 0.62, \quad 8.02, \quad 0.81\} \\
& \bar{P}_{0}=\{\}
\end{aligned}
$$

At the beginning of the algorithm (i.e. when the archive set, $\bar{P}_{0}$ is empty $(\emptyset)$, we do a dominance comparison of all the solutions in the initial parent population, $P_{0}$ as described in detail in section 3.3.1.2 of the NSGA-II algorithm. Hence, applying the non-dominated sorting algorithm to the initial population, $P_{0}$ in equation 3.56 , we have:

$$
\begin{aligned}
& \mathcal{F}_{1}=\{2, \quad 7, \quad 8, \quad 10\} \\
& \mathcal{F}_{2}=\{6\} \\
& \mathcal{F}_{3}=\{4, \quad 9\} \\
& \mathcal{F}_{4}=\{1, \quad 5\} \\
& \mathcal{F}_{5}=\{3\}
\end{aligned}
$$

where, the non-dominated front (or rank) $\mathcal{F}_{1}$ contains solutions in set $P_{0}$ that are ranked the highest among the solutions in the initial parent population, $P_{0}$ in equation 
3.56 .

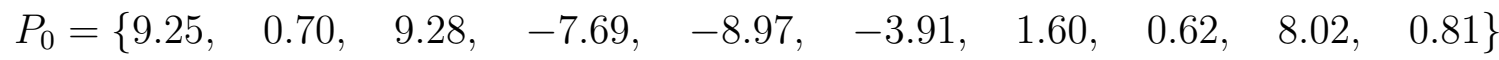

$\bar{P}_{0}=\{0.70, \quad 1.60, \quad 0.62, \quad 0.81\}$

Equation 3.57 shows the highest ranked non-dominated front in the initial parent population (i.e. $\mathcal{F}_{1}$ ). Then we deviate from the NSGA-II and NSGA-III algorithms by copying Solutions $2,7,8$ and 10 and placing them into the empty archive set, $\bar{P}_{0}$ as shown in equation 3.58 .

\subsubsection{Compute fitness value attributes}

Subsequently, two fitness value attributes are computed for solutions in the current parent population, $P_{t}$ and the current archive set, $\bar{P}_{t}$. The first fitness attribute to be computed is the strength value defined as $S(i)$ which represents the number of solutions dominated by the solution $i$ in the combined set of the current parent population, $P_{t}$ and the current archive population, $\bar{P}_{t}$. The strength value is formalized mathematically in equation 3.59 as:

$$
S(i)=\left|\left\{j \mid j \in\left(P_{t} \cup \bar{P}_{t}\right) \cap i \prec j\right\}\right|
$$

where $|\cdot|$ denotes the cardinality of the set and $\prec$ is the dominance relational operator. For our example problem, the current parent and archive population sets are sets $P_{0}$ and $\bar{P}_{0}$ respectively in equation 3.58 . Thus combining both sets, we have:

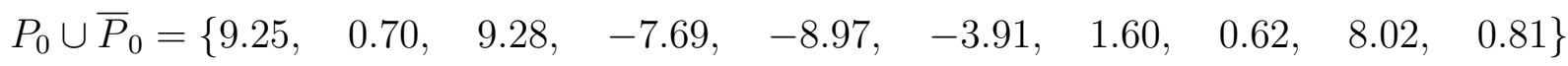


In the first iteration of the algorithm, we recognize that the evaluating the union of sets $P_{0}$ and $\bar{P}_{0}$ in equation 3.60 is going to be the same, because $\bar{P}_{0}$ is a subset of $P_{0}$.

The strength value for Solution 1, S(1) is computed by counting the number of solutions in the combined parent and archive set in equation 3.60 that are dominated by Solution 1. Comparing the objective values of Solution 1 with the objective values of the other solutions in equation 3.61, we can see that Solution 1 only dominates (has smaller function values than) Solution 3. Hence, the strength value for Solution $1, S(1)=1$ as shown in table 3.8 .

$$
\begin{aligned}
& F_{0}=\{(85.56,52.56), \quad(0.49,1.69), \quad(86.12,53.00), \\
& (59.14,93.90), \quad(80.46,120.34), \quad(15.29,34.93), \\
& (2.56,0.16), \quad(0.38,1.90), \quad(64.32,36.24),
\end{aligned}
$$

Likewise, the strength value for Solution $2, S(2)=6$ as shown in table 3.8. This is because Solution 2 dominates Solutions 1, 3, 4, 5, 6 and 9. Computed in a similar way, the strength values for the remaining solutions in the combined population set of equation 3.60 are shown in table 3.8.

The second fitness value to be computed for every solution in the combined population of the current parent and archive sets is the raw fitness value. The raw fitness value is dependent on the earlier computed strength values in table 3.8. The raw fitness value, $R(i)$ for a solution $i$ can be defined as the sum of the strength values assigned to solutions (both in the population and archive sets) that dominate solution $i$.

$$
R(i)=\sum_{j \in\left(P_{t} \cup \bar{P}_{t}\right) \cap j \prec i} S(j)
$$




\begin{tabular}{|c|c|}
\hline Solution 1: 9.25 & Solution 2: 0.70 \\
\hline$S(1): \quad 1$ & $S(2): \quad 6$ \\
\hline Solution 3: 9.28 & Solution 4: $\quad-7.69$ \\
\hline$S(3): \quad 0$ & $S(4): \quad 1$ \\
\hline Solution 5: $\quad-8.97$ & Solution 6: $\quad-3.91$ \\
\hline$S(5): 0$ & $S(6): \quad 5$ \\
\hline Solution 7: 1.60 & Solution 8: 0.62 \\
\hline$S(7): \quad 6$ & $S(8): \quad 6$ \\
\hline Solution 9: 8.02 & Solution 10: 0.81 \\
\hline$S(9):$ & $s(10$ \\
\hline
\end{tabular}

Table 3.8: Strength values for solutions in combined population in equation 3.60

The raw fitness value is expressed mathematically as shown in equation 3.62. The raw fitness value for Solution $1, R(1)=32$ as shown in table 3.9. This is because Solution 1 is dominated by Solutions 2, 6, 7, 8, 9 and 10. Thus adding their strength values together (i.e. $S(2)+S(6)+S(7)+S(8)+S(9)+S(10)$ in table 3.8) gives $R(1)=32$ as shown in table 3.9.

Likewise, the raw fitness value for Solution $2, R(2)=0$. This is because Solution 2 is not dominated by any solution in the combined parent and archive population in equation 3.60. Table 3.9 shows the raw fitness values for all the solutions in the combined population of equation 3.60.

One thing to note from the computed fitness values is that the lower the raw fitness value, the better the solution; for example, if $R(i)=0$ (i.e. it is a nondominated solution). In situations where some solutions do not dominate each other (i.e. they belong to the same non-domination front), an additional density attribute is computed to discriminate between them. To evaluate the density attribute for solution $i$, we simply compute the euclidean distance (in the objective space) to all 


\begin{tabular}{|c|c|}
\hline Solution 1: 9.25 & Solution 2: 0.70 \\
\hline$S(1): \quad 1$ & $S(2): \quad 6$ \\
\hline$R(1): \quad 32$ & $R(2): \quad 0$ \\
\hline Solution 3: 9.28 & Solution 4: $\quad-7.69$ \\
\hline$S(3): \quad 0$ & $S(4): \quad 1$ \\
\hline$R(3): \quad 33$ & $R(4): \quad 29$ \\
\hline Solution 5: $\quad-8.97$ & Solution 6: $\quad-3.91$ \\
\hline$S(5): 0$ & $S(6): \quad 5$ \\
\hline$R(5): \quad 33$ & $R(6): \quad 24$ \\
\hline Solution 7: 1.60 & Solution 8: 0.62 \\
\hline$S(7): \quad 6$ & $S(8): \quad 6$ \\
\hline$R(7): \quad 0$ & $R(8): \quad 0$ \\
\hline Solution 9: 8.02 & Solution 10: 0.81 \\
\hline$S(9): \quad 3$ & $S(10): \quad 6$ \\
\hline$R(9): \quad 29$ & $R(10): \quad 0$ \\
\hline
\end{tabular}

Table 3.9: Raw fitness values for solutions in combined population in equation 3.60 
other solutions in the combined parent population and archive set, and sort them in increasing order of magnitude.

Distance from solution 1 to solution 1 :

$d_{1}^{1}=0$

Distance from solution 1 to solution 2:

$d_{1}^{2}=\sqrt{(0.49-85.56)^{2}+(1.69-52.56)^{2}} d_{1}^{2}=99.12$

$\vdots$

Distance from solution 1 to solution 10 :

$$
\begin{aligned}
& d_{1}^{10}=\sqrt{(0.66-85.56)^{2}+(1.42-52.56)^{2}} \\
& d_{1}^{10}=99.11
\end{aligned}
$$

Thus, to compute the distance between solution $i$ and solution $j$, we apply the euclidean distance formula: $d_{i}^{j}=\sqrt{\left(f_{1}(j)-f_{1}(i)\right)^{2}+\left(f_{2}(j)-f_{2}(i)\right)^{2}}$, where the values of $f_{1}(x)=x^{2}$ and $f_{2}(x)=(x-2)^{2}$ are presented in equation 3.61 in the form $\left(f_{1}(x), f_{2}(x)\right)$. Hence, computing the euclidean distance from Solution 1 in the combined parent population of equation 3.60 to every other solution in the population is shown in equation 3.63.

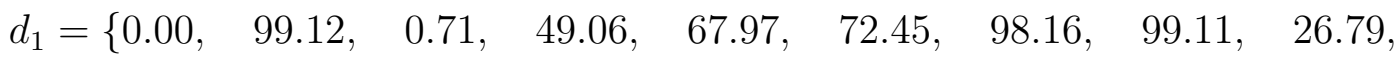

Therefore, the distance from Solution 1 to every other solution is given in equation 3.64. In the same way we compute the distance from each solution to every other solution in the combined population set of equation 3.60. Equation 3.65 shows the euclidean distance set for every solution in the combined population in 3.60 sorted in 
increasing order of magnitude.

$$
\begin{aligned}
& d_{1}=\{0.00, \quad 0.71, \quad 26.79, \quad 49.06, \quad 67.97, \quad 72.45, \quad 98.16, \quad 99.11, \quad 99.11, \quad 99.12\} \\
& d_{2}=\{0.00, \quad 0.24, \quad 0.32, \quad 2.57, \quad 36.39, \quad 72.58, \quad 99.12, \quad 99.83, \quad 109.28, \quad 143.08\} \\
& d_{3}=\{0.00, \quad 0.71, \quad 27.50, \quad 49.00, \quad 67.58, \quad 73.10, \quad 98.87, \quad 99.81, \quad 99.82, \quad 99.83\}
\end{aligned}
$$

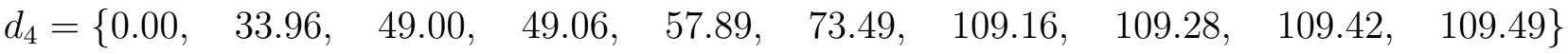

$$
\begin{aligned}
& d_{5}=\{0.00, \quad 33.96, \quad 67.58, \quad 67.97, \quad 85.63, \quad 107.43, \quad 142.97, \quad 143.08, \quad 143.21, \quad 143.22\} \\
& d_{6}=\{0.00, \quad 36.24, \quad 36.39, \quad 36.56, \quad 37.03, \quad 49.05, \quad 72.45, \quad 73.10, \quad 73.49, \quad 107.43\} \\
& d_{7}=\{0.00, \quad 2.28, \quad 2.57, \quad 2.79, \quad 37.03, \quad 71.53, \quad 98.16, \quad 98.87, \quad 109.49, \quad 143.22\} \\
& d_{8}=\{0.00, \quad 0.24, \quad 0.56, \quad 2.79, \quad 36.24, \quad 72.58, \quad 99.11, \quad 99.81, \quad 109.16, \quad 142.97\} \\
& d_{9}=\{0.00, \quad 26.79, \quad 27.50, \quad 49.05, \quad 57.89, \quad 71.53, \quad 72.56, \quad 72.58, \quad 72.58, \quad 85.63\} \\
& d_{10}=\{0.00, \quad 0.32, \quad 0.56, \quad 2.28, \quad 36.56, \quad 72.56, \quad 99.11, \quad 99.82, \quad 109.42, \quad 143.21\}
\end{aligned}
$$

We then take the inverse of the distance to the $k$ th nearest element as the density attribute. As stipulated by the authors of the algorithm, $k$ is defined as the square root of the sample size (i.e. $\sqrt{N+\bar{N}}$ ). Hence, for our example's sample size, $k=$ $\sqrt{10+10}=4$ rounded to the nearest integer. The density value formula is shown in equation 3.66.

$$
D(i)=\frac{1}{\sigma_{i}^{k}+2}
$$

where $\sigma_{i}^{k}$ is the distance of solution $i$ to the nearest $k$ th element and 2 is added to ensure the density value is positive and larger than 0. Finally, the total fitness value of each solution is given in equation 3.67

$$
F(i)=R(i)+D(i)
$$


Thus computing the density value, $D(i)$ and the total fitness value, $F(i)$ for each solution in the combined population, we have:

For solution 1:

$$
\begin{aligned}
& \sigma_{1}^{4}=d_{1}(4)=49.06 \\
& D(1)=\frac{1}{49.06+2}=0.02 \text { from equation } 3.66 \\
& F(1)=32+0.02=32.02 \text { from equation } 3.67 \\
& \vdots
\end{aligned}
$$

For solution 10:

$$
\begin{aligned}
& \sigma_{10}^{4}=d_{10}(4)=2.28 \\
& D(10)=\frac{1}{2.28+2}=0.23 \\
& F(10)=0+0.23=0.23
\end{aligned}
$$

Therefore, the distance values and the total fitness values are presented in equation 3.69 below.

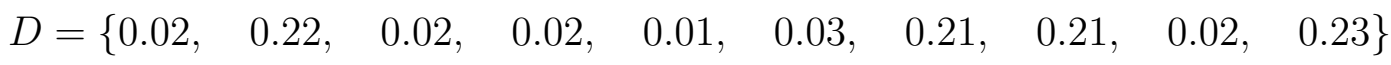
$F=\{32.02, \quad 0.22, \quad 33.02, \quad 29.02, \quad 33.01, \quad 24.03, \quad 0.21, \quad 0.21, \quad 29.02, \quad 0.23\}$

\subsubsection{Environmental selection process}

The next step is the selection process known as environmental selection. The first step in this process is to copy all the non-dominated solutions (i.e. solutions with total fitness values, $F(i)$ lower than 1 ) in the population and archive sets $P_{t}$ and $\bar{P}_{t}$ 
respectively into the next generation's archive set $\bar{P}_{t+1}$ as shown in equation 3.70.

$$
\bar{P}_{t+1}=\left\{i \mid i \in\left(P_{t} \cup \bar{P}_{t}\right) \cap F(i)<1\right\}
$$

Thus, the next generation's archive set in our example problem, $\bar{P}_{1}$ will consist of the solutions in equation 3.69 whose total fitness values is less than 1 (i.e. $F(i)<1$ ). Therefore, $\bar{P}_{1}$ will consist $F(2), F(7), F(8)$ and $F(10)$ which correspond to Solutions 2, 7, 8 and 10 from equation 3.60 as shown below:

$$
\bar{P}_{1}=\{0.70, \quad 1.60, \quad 0.62, \quad 0.81\}
$$

If the number of the non-dominated solutions from both sets (population and archive) is equal to the size $\bar{N}$ of the next generation's archive set, the selection process is complete, otherwise we can have two difference scenarios.

One scenario is if the size of the current archive set $\bar{P}_{t+1}$ is populated with the non-dominated solutions from the previous combined population and archive set is less than the predefined size $\bar{N}$. In this case, we sort the combined archive and population solutions in ascending order of magnitude of their fitness values $F(i)$ and pick the best $\bar{N}-\left|\bar{P}_{t+1}\right|$ solutions. For our example problem, the size of the current archive set $\bar{P}_{1}$ is less than the predefined size $\bar{N}=10$. Therefore, we sort set $F$ in equation 3.60 in ascending order of magnitude and choose the best 6 solutions which results back in the parent population as shown below:

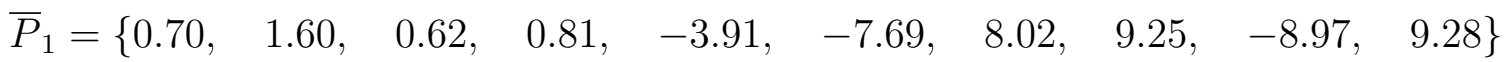

The second scenario is when the size of the current archive set exceeds $\bar{N}$. In this case, a truncation operation is invoked to prune the solutions leaving only the 
best ones. The truncation process is an iterative one where a solution which has the smallest distance to another solution is selected at each iteration and if we have a tie between two or more solutions, the second smallest distance is considered and the procedure is repeated till the next generation's archive set is filled.

If the termination criteria (which is the maximum number of generations in this thesis) is satisfied, we take the current archive population set as the pareto-optimal solution. Otherwise, we apply the usual genetic operators to create an offspring population and repeat the following steps: perform non-dominated sorting on the new internal population and add the solutions in the first non-dominated front to the current archive set as described in section 3.3.3.1; then we compute the fitness value attributes of all the solutions in the external archive set as described in section 3.3.3.2: if the size of the external archive set exceeds the predefined size, we truncate the set using the fitness value attributes as a metric.

Figure 3.20 shows the pareto-optimal front for the solution to the example problem in equation 3.2, where the population size for both the internal and external archive set is 100 and the algorithm terminated after 100 iterations. In figure 3.20, the axes represent the two objective function values in the multi-objective problem in equation 3.2 and the points on the graph represent solutions in the final population. The SPEA2 algorithm is summarized in algorithm 6 . The algorithm has a computational complexity of $O\left(M P^{2}\right)$ where $M$ is the number of objectives and $P=N+\bar{N}$ is the combined population size for the parent and archive populations. 


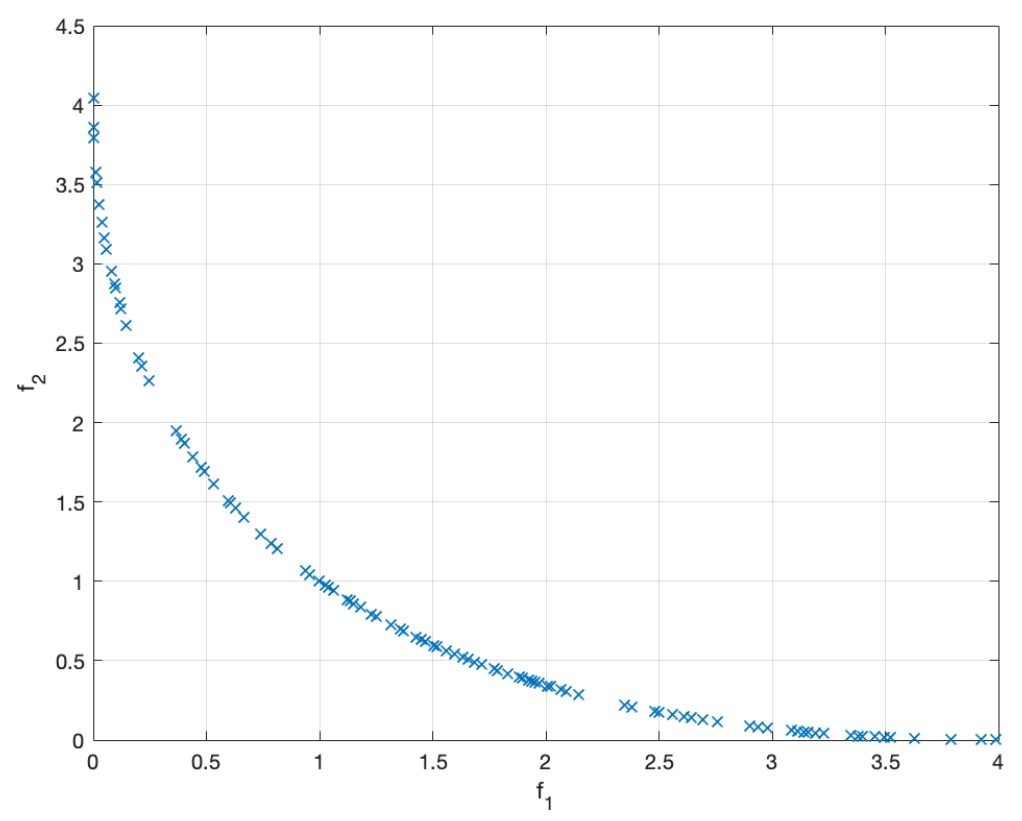

Figure 3.20: Pareto-optimal front showing solution to equation 3.2 using SPEA2 algorithm

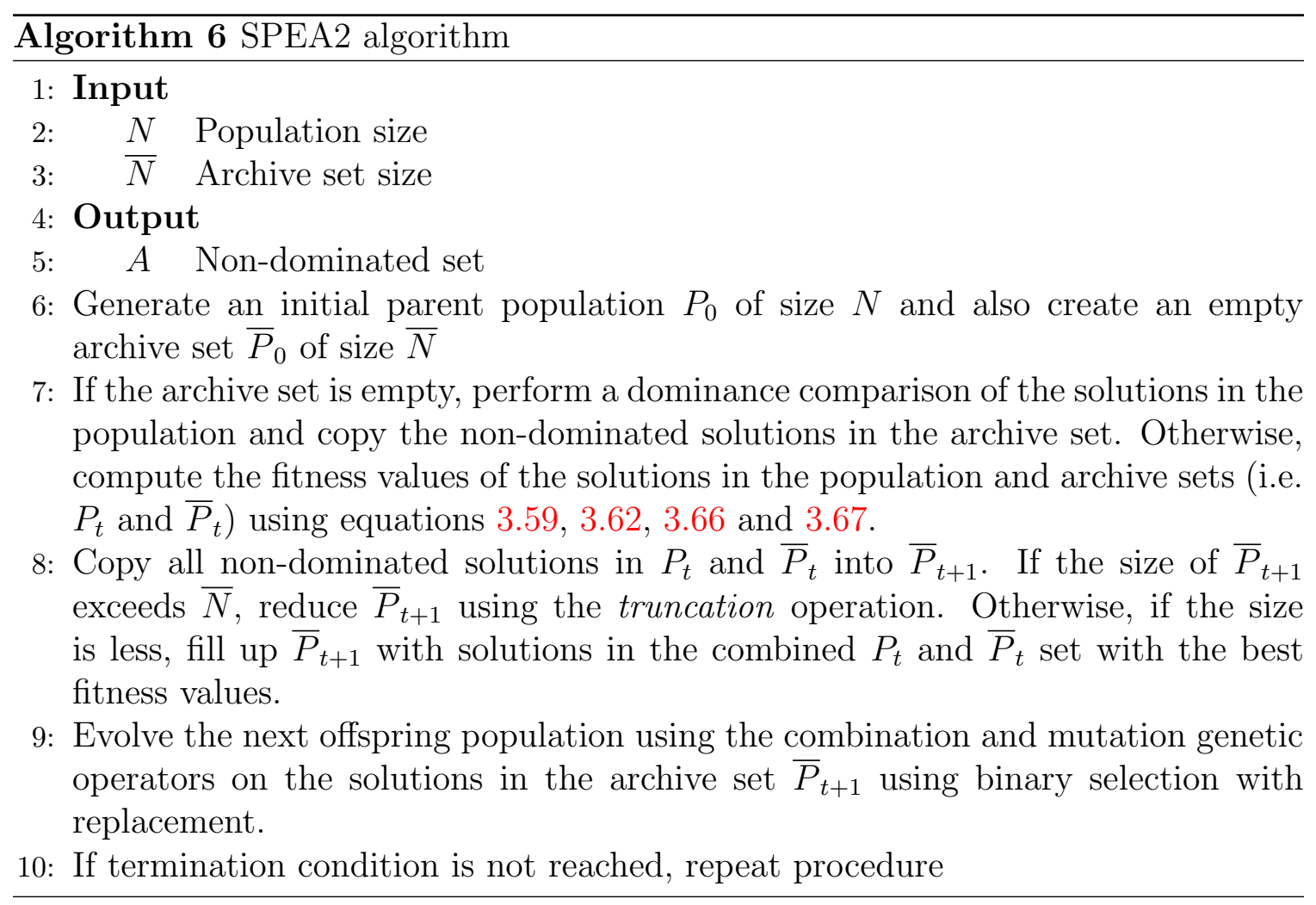




\subsubsection{Region-based Pareto Envelope-based Selection Algo- rithm (PESA-II)}

The improved region-based pareto envelope-based selection algorithm (PESA-II) introduced by Corne et al in [33] is another efficient multi-objective evolutionary algorithm which proposes a new selection and diversity maintenance technique which moves away from the traditional ways of selection operation based on the fitness of individual solutions in the population to one based on the fitness of hyperboxes (occupied by at least one solution in the population) in the objective space. A high level description of the algorithm is as follows.

\subsubsection{Initialization}

The first step in the algorithm procedure (as with all other evolutionary algorithms) is to generate an initial internal parent population, $I P_{0}$, of size $P_{I}$ and an empty external archive population set, $E P_{0}$ with maximum size set to $P_{E}$. Once again we will start with the same parent population as we did in the NSGA-II, NSGA-III and SPEA2 algorithms, where we have the randomly initialized parent population shown in equation 3.73 for the multi-objective problem in equation 3.2 ; where $I P_{0}$ is of size $P_{I}=10$ and the empty archive set, $E P_{0}$ is predefined with a maximum size of $P_{E}=10$.

$$
\begin{aligned}
& I P_{0}=\{9.25, \quad 0.70, \quad 9.28, \quad-7.69, \quad-8.97, \quad-3.91, \quad 1.60, \quad 0.62, \quad 8.02, \quad 0.81\} \\
& E P_{0}=\{\}
\end{aligned}
$$

The first time through the algorithm (i.e. when the size $P_{E}$ of the external (archive) set is empty), we perform a dominance comparison of all the solutions 
in the internal population (as described in detail in section 3.3.1.2 of the NSGA-II algorithm) and copy the non-dominated solutions into the external (archive) set. Once again, applying the non-dominated sorting algorithm to the initial population, $I P_{0}$ in equation 3.73 , we have:

$$
\begin{aligned}
& \mathcal{F}_{1}=\{2, \quad 7, \quad 8, \quad 10\} \\
& \mathcal{F}_{2}=\{6\} \\
& \mathcal{F}_{3}=\{4, \quad 9\} \\
& \mathcal{F}_{4}=\{1, \quad 5\} \\
& \mathcal{F}_{5}=\{3\}
\end{aligned}
$$

where, the non-dominated front (or rank) $\mathcal{F}_{1}$ contains solutions in set $I P_{0}$ that are ranked the highest among the solutions in the initial parent population in equation 3.73 .

$$
\begin{aligned}
& I P_{0}=\{9.25, \quad 0.70, \quad 9.28, \quad-7.69, \quad-8.97, \quad-3.91, \quad 1.60, \quad 0.62, \quad 8.02, \quad 0.81\} \\
& E P_{0}=\{0.70, \quad 1.60, \quad 0.62, \quad 0.81\}
\end{aligned}
$$

The highest ranked non-dominated solutions in the initial parent population, $I P_{0}$ (i.e. the first non-domination rank or front, $\mathcal{F}_{1}$ in equation 3.74). Hence, Solutions 2, 7, 8 and 10 are copied into the empty archive set, $E P_{0}$ as shown in equation 3.75 .

If the predefined termination criterion has been reached (unlikely to happen in the first generation), we stop the algorithm and return the set of solutions in the external archive set as the resulting pareto-optimal solutions found so far. Otherwise, we discard the current internal population (i.e. $I P_{0}$ in equation 3.75) and construct a hyper grid for the solution in the current external population (i.e $E P_{0}$ in equation 
3.75) to help with the selection operation.

\subsubsection{Construct a hyper-grid}

The selection operation introduced by the authors in [33] is based on a region-based crowding technique. The crowding strategy works by forming a hyper-grid which divides the normalized objective space into hyper boxes as shown in figure 3.21. The

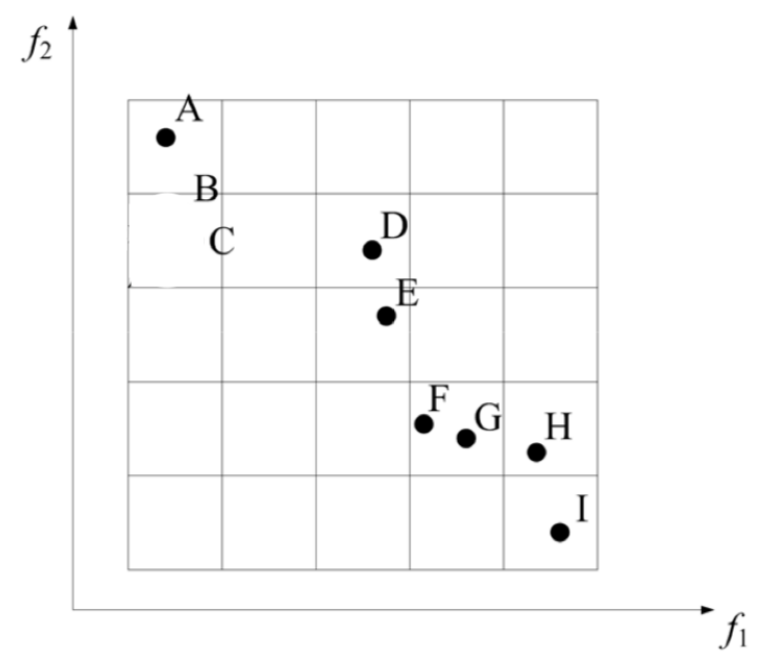

Figure 3.21: Illustration of the crowding hyperbox grid in a 2-objective problem [37]

hyperbox grid is used as a framework to determine the location of individual solutions in the objective space based on the location of the hyperbox it occupies. We adopt the adaptive grid construction technique proposed by Knowles et al in [38] to generate the hyper boxes. The hyperbox generation procedure is as follows:

a Set the number of divisions $(D)$ we want the objective space to be subdivided into.

One thing to note is that, the more boxes in the objective space, the lower the number of solutions in a given box. Accordingly, if $D$ is defined to be 5 divisions, the objective space will be divided into $D^{M}$ hyperboxes, where $M$ is the number of objectives. For our example problem in equation 3.2, we arbitrarily set the number 
of divisions to 7 . Hence, we will have $7^{2}=49$ hyperboxes in our objective space.

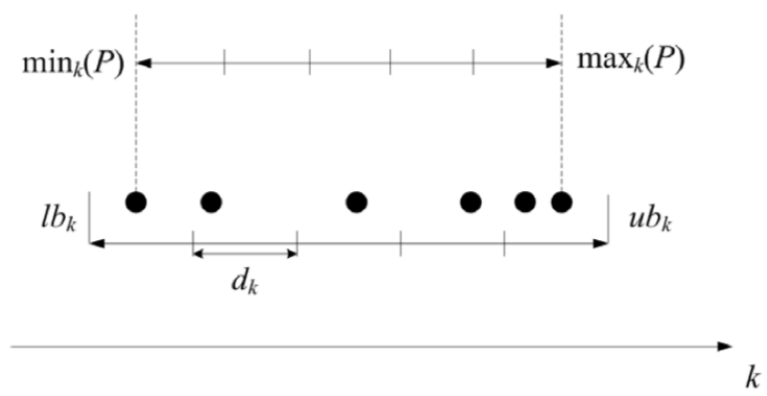

Figure 3.22: Illustration of a $k$ th objective grid construction [37]

b To construct the hyper grid, we take note of the smallest and largest function values (boundary values) in each objective of the archive population and use them to compute the upper and lower boundaries of the hyper-grid. For example, for objective $k$ :

$$
\begin{array}{ll}
\text { lower boundary: } \quad l b_{k}=\min _{k}(P)-\frac{\max _{k}(P)-\min _{k}(P)}{2 \times D} \\
\text { upper boundary: } \quad u b_{k}=\max _{k}(P)+\frac{\max _{k}(P)-\min _{k}(P)}{2 \times D}
\end{array}
$$

where $\min _{k}(P)$ and $\max _{k}(P)$ are the boundary function values of objective $k$ for solution $P$ as depicted in figure 3.22. Equation 3.77 shows the objective function evaluations for our external population in equation 3.75 ; where $f_{1}=x^{2}$ and $f_{2}=$ $(x-2)^{2}$.

$$
\begin{aligned}
& f_{1}(x)=\left\{\begin{array}{llll}
0.49, & 2.56, & 0.38, & 0.66
\end{array}\right\} \\
& f_{2}(x)=\left\{\begin{array}{llll}
1.69, & 0.16, & 1.90, & 1.42
\end{array}\right\}
\end{aligned}
$$

From equation 3.77, we can see that the boundary function values for the first objective (i.e. $\left.f_{1}(x)\right)$ are $\min _{1}(P)=0.38$ and $\max _{1}(P)=2.56$, while the boundary 
functions for the second objective (i.e. $f_{2}(x)$ ) are $\min _{2}(P)=0.16$ and $\max _{2}(P)=$ 1.90. Therefore, the lower and upper boundary values for both objectives are computed as shown below:

Objective 1:

$$
\begin{aligned}
& \text { lower boundary: } \quad l b_{1}=0.38-\frac{2.56-0.38}{2 \times 7}=0.22 \\
& \text { upper boundary: } \quad u b_{1}=2.56+\frac{2.56-0.38}{2 \times 7}=2.72
\end{aligned}
$$

Objective 2:

$$
\begin{array}{ll}
\text { lower boundary: } \quad l b_{2}=0.16-\frac{1.90-0.16}{2 \times 7}=0.04 \\
\text { upper boundary: } \quad u b_{2}=1.90+\frac{1.90-0.16}{2 \times 7}=2.02
\end{array}
$$

c The hyperbox width (as shown in figure 3.22) in the $k$ th objective space is thus computed as:

$$
d_{k}=\frac{u b_{k}-l b_{k}}{D}
$$

Hence, the width of the hyperbox along the $f_{1}$ and $f_{2}$ objectives is computed as shown in equation 3.80 .

\section{Objective 1:}

$$
d_{1}=\frac{2.72-0.22}{7}=0.36
$$

Objective 2:

$$
d_{2}=\frac{2.02-0.04}{7}=0.28
$$

d Finally, the grid coordinate for any solution $A$ in the $k$ th objective can be determined as:

$$
G_{k}(A)=\frac{F_{k}(A)-l b_{k}}{d_{k}}
$$


where $F_{k}(A)$ is the $k$ th objective value of solution $A$. Hence, if we have a twoobjective problem as shown in figure 3.21, we'll have the computed grid coordinates in the form $\left(G_{f_{1}}(A), G_{f_{2}}(A)\right)$. The grid coordinates for the external population members in equation 3.75 are computed as shown in equation 3.82. Hence, the grid coordinates for the solutions in the external population are as follows: Solution 1:(0.75, 5.89), Solution 2:(6.50, 0.43), Solution 3:(0.44, 6.64) and Solution 4:(1.22, 4.93) as computed in equation 3.82. Figure 3.23 shows a graphical depiction of the hyper grid and the solutions of the external population.

Solution 1: 0.70

$$
\begin{aligned}
& G_{1}(1)=\frac{0.49-0.22}{0.36}=0.75 \\
& G_{2}(1)=\frac{1.69-0.04}{0.28}=5.89
\end{aligned}
$$

Solution 2: 1.60

$$
\begin{aligned}
& G_{1}(2)=\frac{2.56-0.22}{0.36}=6.50 \\
& G_{2}(2)=\frac{0.16-0.04}{0.28}=0.43
\end{aligned}
$$

Solution 3: 0.62

$$
\begin{aligned}
& G_{1}(3)=\frac{0.38-0.22}{0.36}=0.44 \\
& G_{2}(3)=\frac{1.90-0.04}{0.28}=6.64
\end{aligned}
$$

Solution 4: 0.81

$$
\begin{aligned}
& G_{1}(4)=\frac{0.66-0.22}{0.36}=1.22 \\
& G_{2}(4)=\frac{1.42-0.04}{0.28}=4.93
\end{aligned}
$$




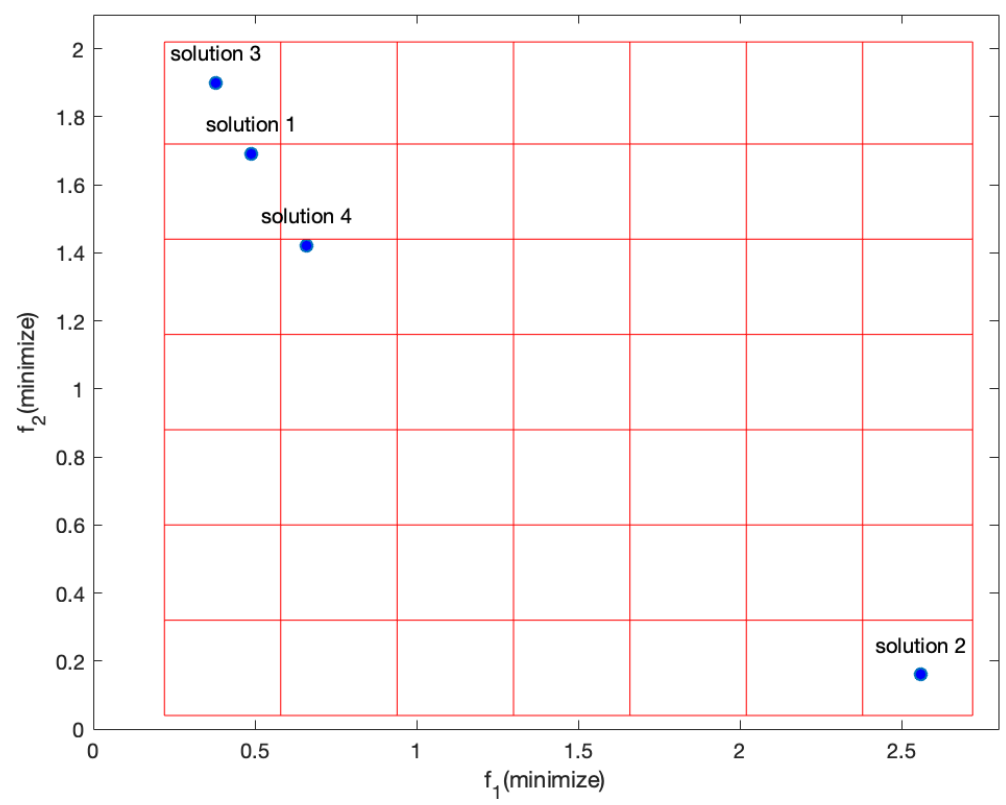

Figure 3.23: Graphical illustration of the hyper-grid for the external population in equation 3.75

\subsubsection{Create new internal population}

After developing the hyperboxes, fitness values are assigned to occupied hyperboxes as opposed to the traditional way of assigning fitness values to individual solutions in the population. The unit of selection is now based on the fitness values of the hyperboxes rather than the fitness values of individual solutions. Therefore, using any standard method (e.g. the tournament selection method introduced in chapter 2), a hyperbox is chosen and an individual solution (for the crossover and mutation operations) is chosen at random from the selected hyperbox. To aid diversity of solutions on the pareto front, we define our fitness evaluation to be of the form:

$$
\frac{1}{n_{h}}
$$


where $n_{h}$ is the number of solutions in hyperbox $h$. Applying this analogy to figure 3.21, we can say the hyperbox containing solution $I$ will have a better fitness to that containing solutions $F$ and $G$. From figure 3.23, we can see that there are only four occupied hyperboxes, namely: hyperboxes 7, 30, 36 and 43 each with a fitness value of $\frac{1}{1}=1$ (since they each contain one solution).

For the selection operation, we apply the tournament selection detailed in chapter 2 to the occupied hyperboxes and select two parents from the external population to create a new offspring by crossover and mutation operations as discussed in chapter 2. The internal population, $I P$ is cleared and the new offspring is then added to the new internal population. We again randomly choose one of the solutions in the external population, mutate it and add it to the new internal population. We continue repeating the selection and mutation operations until the new internal population if filled.

We then perform non-dominated sorting of both the new internal and the external archive populations and populate the external archive population with the highest ranked solutions. Hence, a solution from the internal population can only be copied into the external population set if it is non-dominated by any current solutions in both the internal and external archive sets and all solutions it dominates in the archive set are discarded.

In doing this, situations will arise where the incoming solutions are equally nondominated by the solutions in the external archive set and temporarily renders the external archive over-full (i.e. exceeding the maximum size of $E P_{t}$. The choice of the solutions to remove is by identifying the hyperbox(es) with the largest number of solutions and arbitrarily removing one from each until we reach the number of solutions to keep the predefined set size of the external archive population.

Figure 3.24 shows the pareto-optimal front for the solution to the example problem 
in equation 3.2, where the population size for both the internal and external archive set is 100 and the algorithm terminated after 100 iterations. In figure 3.24, the axes represent the two objective function values in the multi-objective problem in equation 3.2 and the points on the graph represent solutions in the final population. The PESAII algorithm can be summarized in algorithm 7. The algorithm has a computational complexity of $O\left(M P^{2}\right)$ where $M$ is the number of objectives and $P=N+\bar{N}$ is the combined population size of the internal and archive populations.

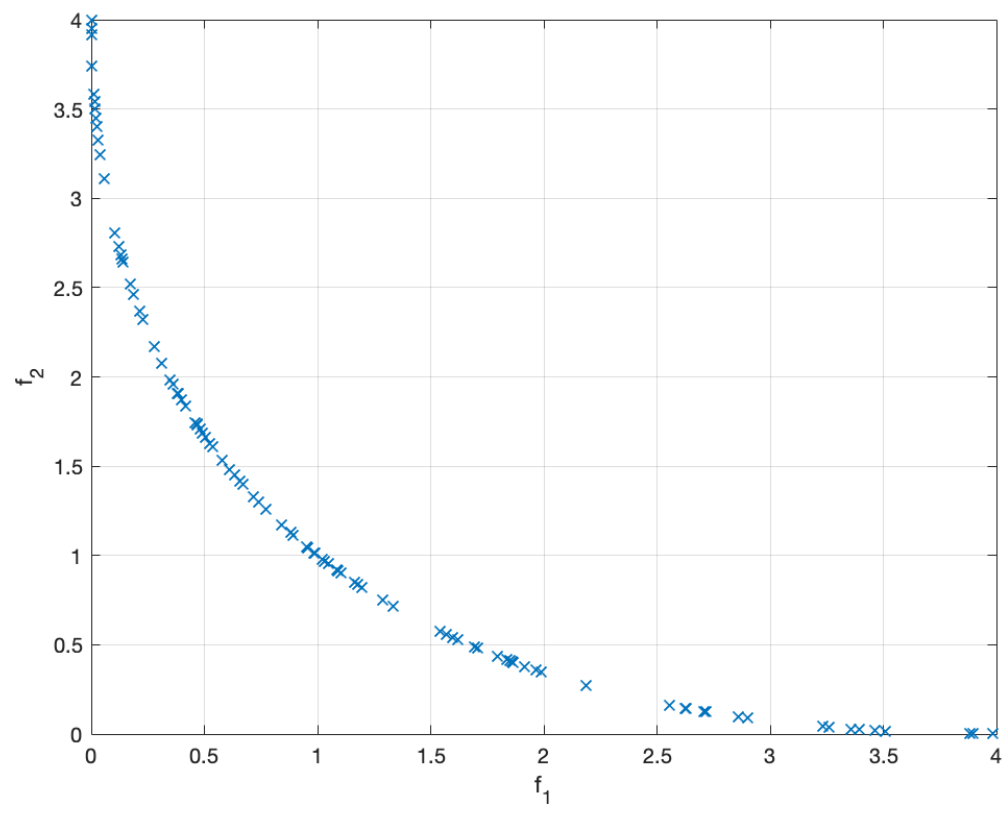

Figure 3.24: Pareto-optimal front showing solution to equation 3.2 using PESA-II algorithm 


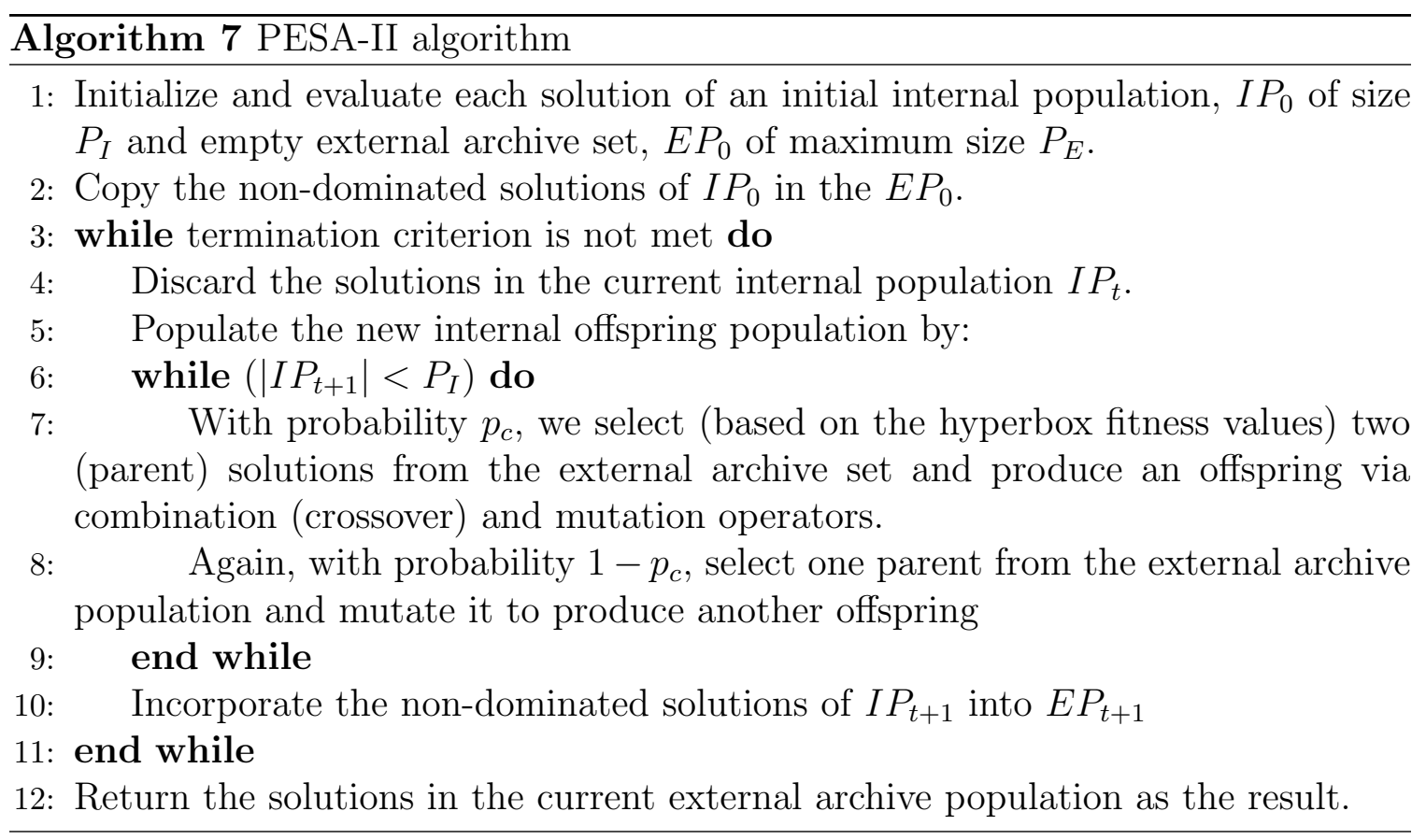

\subsection{Comparing the algorithms}

\subsubsection{Performance of the algorithms}

The algorithms described in this chapter are four of the highest performing evolutionary algorithms at the time this research was carried out. The four algorithms were chosen because of they are designed to enhance exploration with their diversity preservation techniques and proven ability to converge to a set of pareto-optimum solutions. The algorithms cannot be objectively compared as their performance varies depending on the multi-objective problem, nonetheless, their performance in solving a multi-objective problem can be compared.

The metric of comparison employed in this thesis is the hypervolume metric. The hypervolume metric computes the space dominated by the final approximation set obtained by the evoluationary algorithm as shown in figure 3.25. The volume is 
bounded by a reference point which is usually set by computing the worst-case objective value for each objective of the reference set plus some fixed increment. Adding

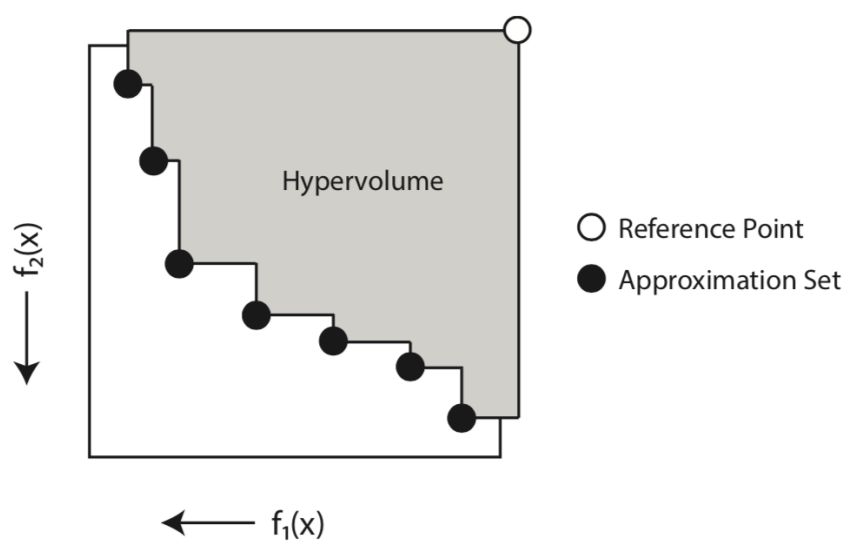

Figure 3.25: Graphical illustration of a hypervolume

the fixed increments allows for the extreme points in the final approximate set to also contribute to the hypervolume. Knowles and Corne et al in [39] recommend using the hypervolume comparison metric because it is scale independent, intuitive and can reflect the degree of performance between two approximate sets.

For our example problem in equation 3.2, the result for computing and comparing the hypervolume metric for the final approximate sets for the four algorithms 
introduced earlier is shown in equations $3.84-3.87$.

NSGA-II :

Hypervolume:

Min: $\quad 0.82865$

Median: 0.82910

Max: 0.82941

Count: 50

Indifferent: []

NSGA-III :

Hypervolume:

Min: $\quad 0.82810$

Median: 0.82812

Max: 0.82813

Count: 50

Indifferent:

PESA-II :

Hypervolume:

Min: $\quad 0.82879$

Median: 0.82965

Max: 0.83014

Count: 50

Indifferent: [SPEA2] 
SPEA2 :

Hypervolume:

Min: $\quad 0.82939$

Median: 0.82958

Max: $\quad 0.82974$

Count: 50

Indifferent: $\quad[\mathrm{PESA}-\mathrm{II}]$

The equations in 3.84 - 3.87 show the minimum, median and maximum hypervolume value achieved by each algorithm. Each algorithm is executed for 10,000 number of function evaluations (NFE) each which is the number of times the objective functions are evaluated. The count field shows how many times each algorithm is run for, while the indifferent field shows the algorithm of which there is statistical indifference with 95\% confidence level. Hence, the performance of the SPEA2 and PESA-II algorithms are identical or not statistically different.

Figure 3.26 shows a graphical illustration of the hypervolume metric computation. The result of each algorithm is presented as a coloured box. The filled, rectangular boxes show the range in the $25 \%$ to $75 \%$ percentile (i.e. the middle $50 \%$ of the solutions) and the horizontal line shows the median. The thin lines at the top and bottom of the rectangular box show the regular min and max of the data and finally, the solid black circle shows the mean. Using the plot in figure 3.26, we can see quickly that though the performance of the four algorithms are very close, the PESAII algorithm seems to perform best (because larger values are preferred) followed by the SPEA2 algorithm and that the NSGA-III algorithm has the worst performance. 


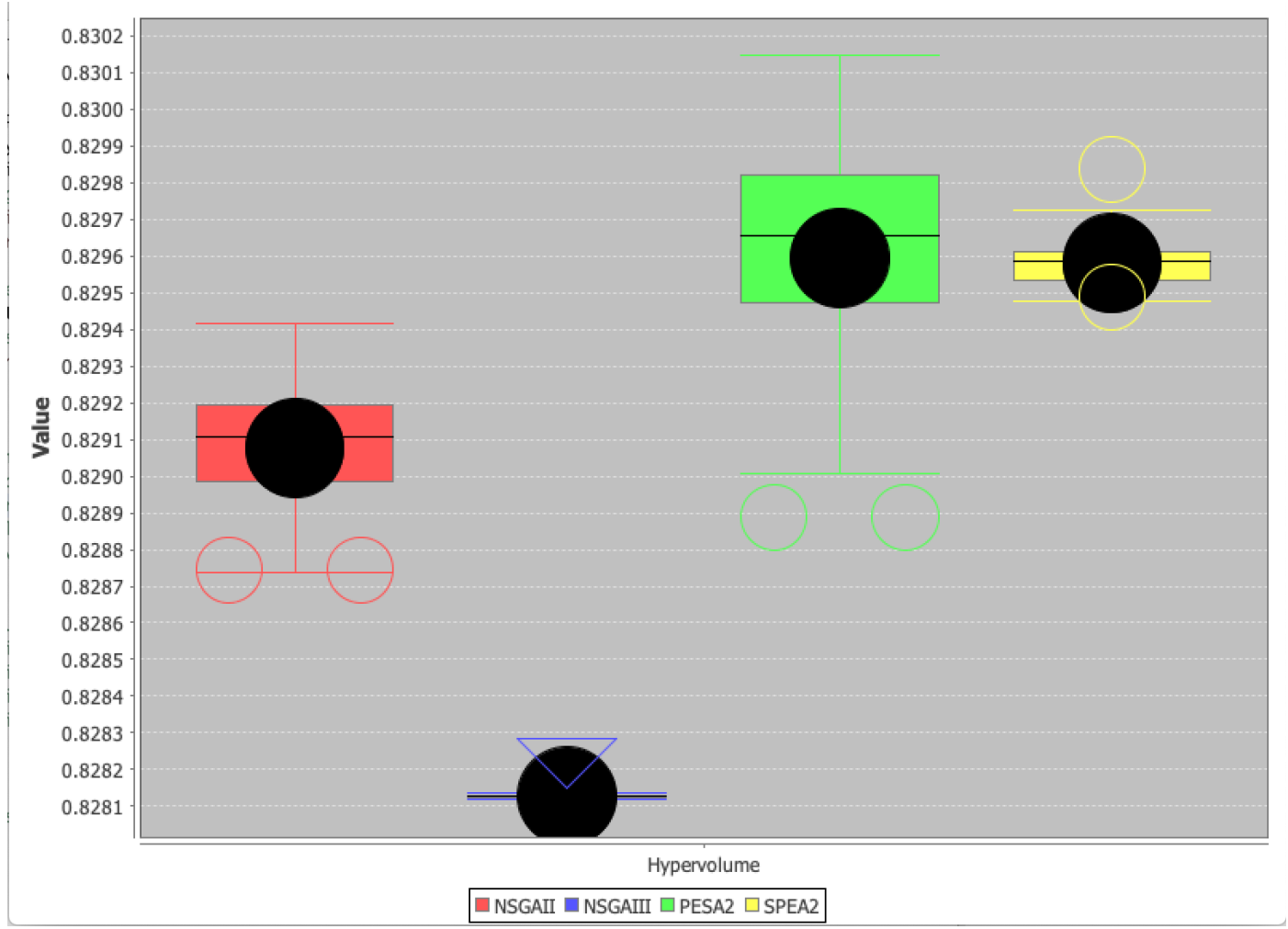

Figure 3.26: Graphical plot of the hypervolume metric 
Previously, we statistically compared the end-of-run performance of the four algorithms using only the final pareto approximation sets. Now we take a different approach by investigating the intermediate performances of the algorithms; that is, we want to see how the performance of the algorithms evolve over time.

We once again employ the hypervolume metric to compute the volume of space dominated by each generation's approximation generation set bounded by a reference point. For our example we needed to generate a reference set, to do this, we solved the multi-objective problem 50 times for each algorithm and merged the results in to a reference set. We can see from figures 3.27 - 3.30 that the four algorithms perform similarly well during the course of the tests.

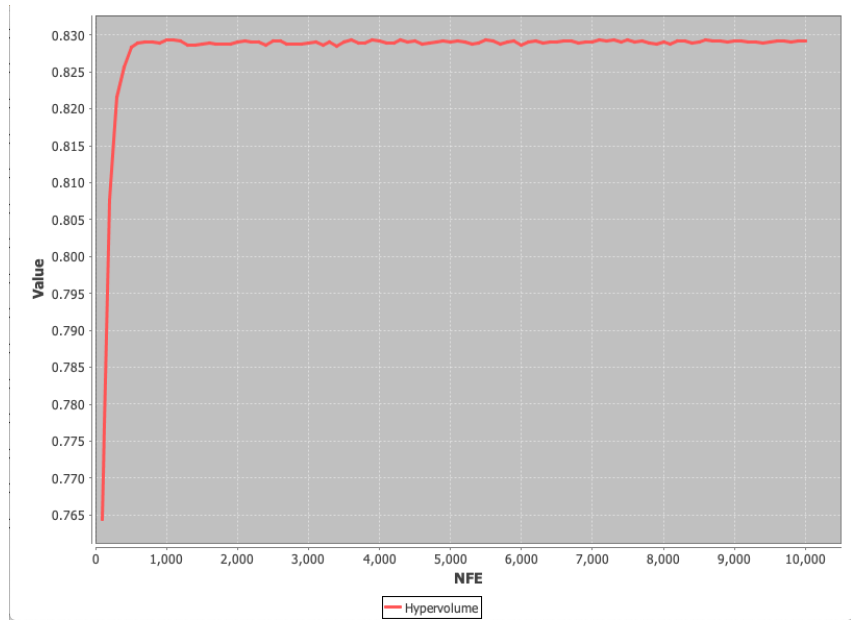

Figure 3.27: Hyper volume runtime dynamics for the NSGA-II algorithm

\subsubsection{Effects of the diversity operations of the algorithms}

According to how the algorithms are designed, the diversity operations which constitute the major differences between the algorithms usually come into play when the 


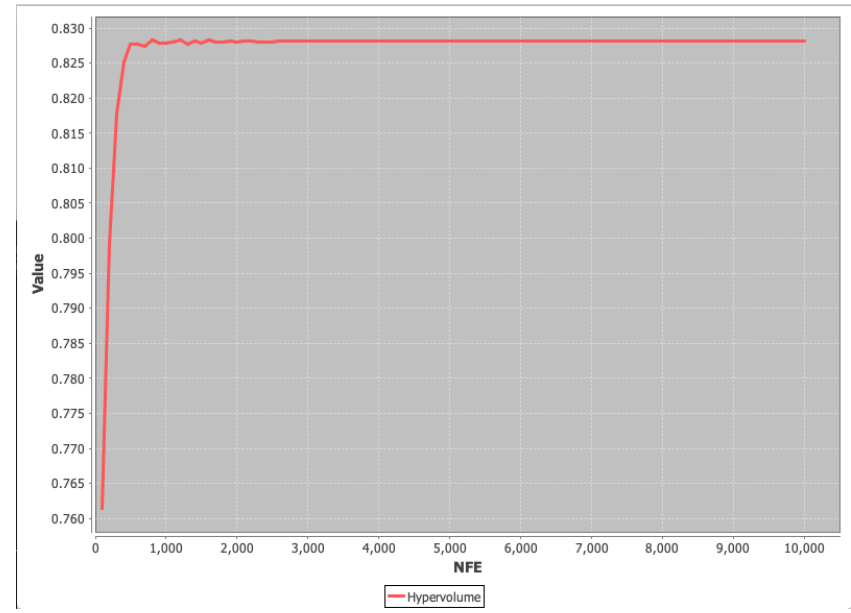

Figure 3.28: Hyper volume runtime dynamics for the NSGA-III algorithm

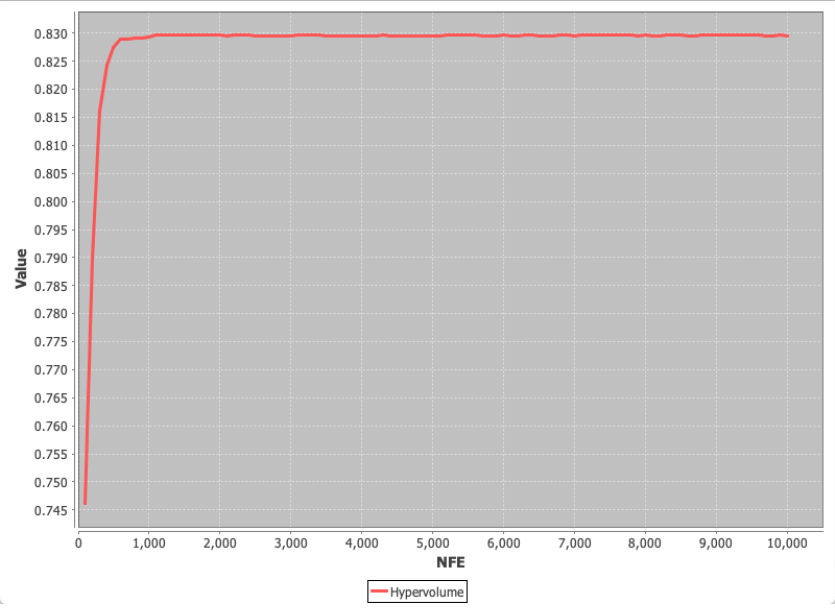

Figure 3.29: Hyper volume runtime dynamics for the SPEA2 algorithm

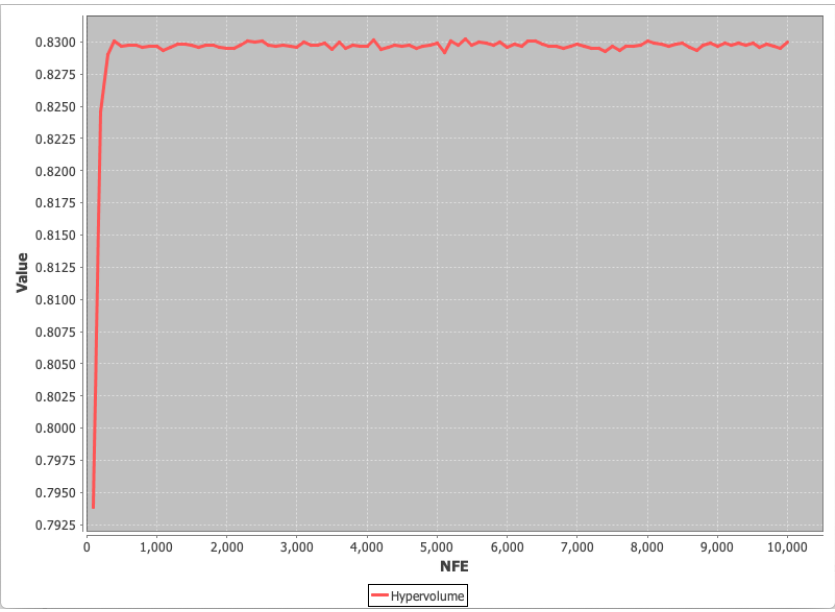

Figure 3.30: Hyper volume runtime dynamics for the PESA-II algorithm 
algorithms are trying to fill up or truncate the members of the next generation's parent population. As such, we observed that the contributions of the diversity operations of each algorithm are minimal.

To show this, we ran each algorithm 100 times (where each run is for 100 iterations) for a population size of 100 each and computed the average number of times (to the nearest whole number) the diversity operations of each algorithm influenced the new generation's population as shown in table 3.10. We can see from the table that the crowding-distance diversity operation of the NSGA-II algorithm came into play an average of 37 times out of 10,000 iterations (i.e. $100 \times 100$ iterations), while the reference point-based diversity operation of the NSGA-III algorithm came into effect 41 times out of 10,000 iterations.

Table 3.10: Average diversity operation count

\begin{tabular}{|c|c|c|c|c|}
\hline Algorithm & NSGA-II & NSGA-III & SPEA2 & PESA-II \\
\hline Average count & 37 & 41 & 39 & 35 \\
\hline
\end{tabular}

The next test we carried out was to run another simulation of the algorithms using the same initial parent population for each and forcing the same selection, crossover and mutation operations at every generation. We did this to study the effect of the diversity preservation operation of each algorithm on a small population size. From the simulation test, we observed that the four algorithms produce the same results as shown in equation 3.88.

$$
\{-0.00018, \quad 2.00002, \quad 0.48783, \quad 1.00920, \quad 1.48102\}
$$

Figure 3.31 shows the pareto front showing the approximate solutions. Figure 3.32 also shows another pareto front result (out of many) using the same small population 
size of 5 . One thing we observed was that the algorithms naturally resulted in diverse solutions due to the stochastic nature of the algorithms. Hence, we can conclude that the diversity operations of the algorithms do not come into play when the population size is small.

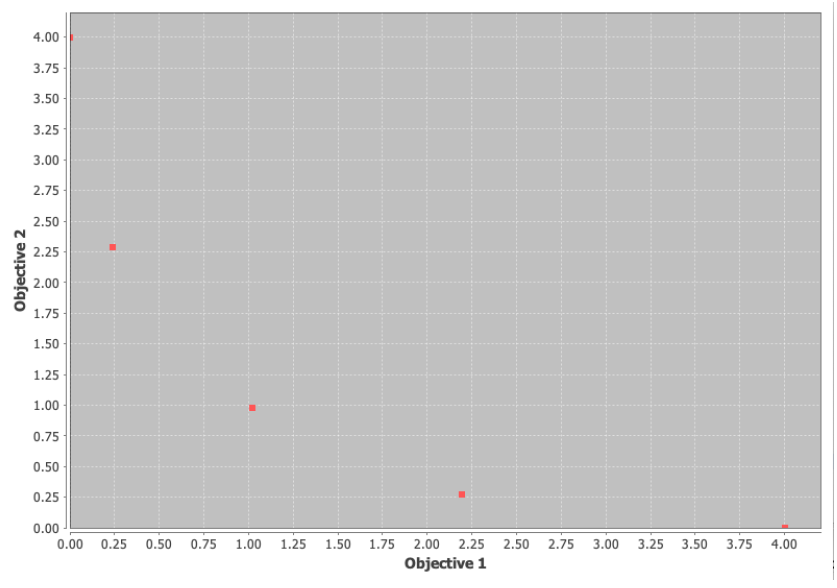

Figure 3.31: Pareto front for the 4 algorithms using a small population size

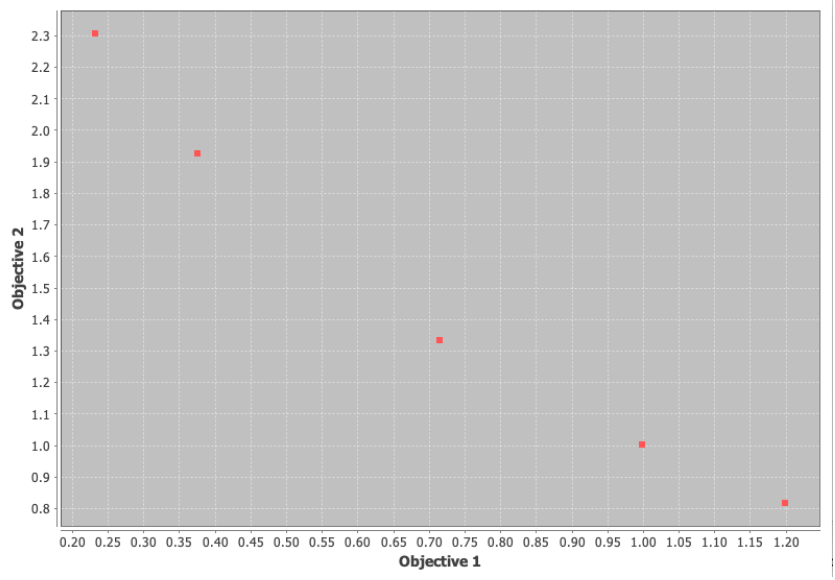

Figure 3.32: Another pareto front using a small population size 


\section{Chapter 4}

\section{Simulation Analysis and Results}

\subsection{Introduction}

In this chapter, we are going to look into our application of interest: the illegal, unreported and unregulated (IUU) fishing incident which is a maritime domain awareness problem.We present the results of the simulations conducted to demonstrate that multi-objective evolutionary algorithms can be applied to IUU fishing. The four multi-objective evolutionary algorithms (MOEAs) discussed in chapter 3 were employed in generating potential responses to pre-detected illegal, unreported and unregulated (IUU) fishing events.

\subsection{Generating potential responses to IUU fishing events with MOEAs}

For this simulation, we set out to demonstrate a methodology employing MultiObjective Evolutionary Algorithms (MOEAs) to automatically generate a paretooptimal set of potential promising candidate responses on the account that an Illegal, Unreported and Unregulated fishing fishing event has been detected. A candidate 
response consists of a set of assets (e.g. boats, helicopters etc.). To this end, we modelled the potential response as a multi-objective numerical problem and solved the problem using the four Evolutionary Multi-Objective Optimization algorithms discussed in chapter 3 .

\subsubsection{The IUU scenario}

The simulation scenario is set in the Lobster Fishing Areas (LFA) ${ }^{1}$ of the ScotiaFundy Fisheries Maritime Region on the East coast of Canada as shown in figure 4.1 which shows the initialized synthetic IUU scenario. From figure 4.1, the sus-

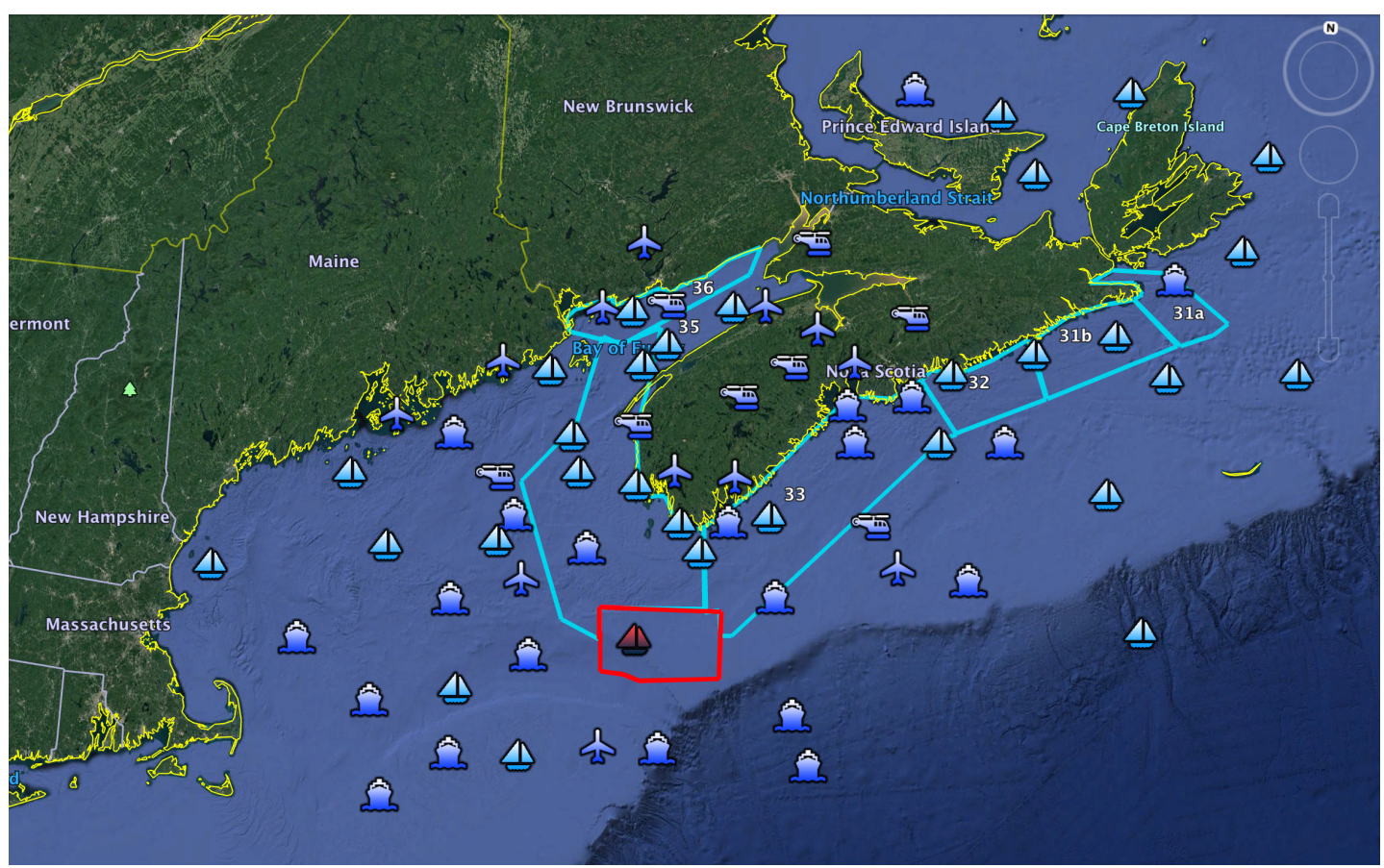

Figure 4.1: Initial IUU Scenario

pected IUU fishing vessel is located in $L F A-40$ (the red boat in red zone) which is

\footnotetext{
${ }^{1} \mathrm{http}$ ///www.dfo-mpo.gc.ca/fm-gp/sustainable-durable/fisheries-peches/lobster-homardeng.htm
} 
closed to inshore-offshore lobster fishing as a conservative measure [40]. The initial IUU scenario depicts the Automatic Identification System (AIS) Gaps scenario which describes a situation where suspected fishing vessels turn off their transponders at intervals to avoid reporting their geographical location especially when fishing in restricted zones as shown in figure 4.1.

Located around the area are 72 arbitrary assets consisting of life boats, law enforcement boats, surveillance aerial vehicles (light aircrafts, helicopters, UAVs etc). which we assume to be located at these locations at the time of simulation and any combination of these assets can be a potential response. Table 4.1 outlines the as-

Table 4.1: Asset Features

\begin{tabular}{|c|c|c|c|c|}
\hline Asset & Number & $\begin{array}{c}\text { Moving } \\
\text { Cost }(\$ / \mathrm{km})\end{array}$ & $\begin{array}{c}\text { Asset } \\
\text { Speed }(\mathrm{km} / \mathrm{hr})\end{array}$ & $\begin{array}{c}\text { Visibility } \\
\text { Radius (m) }\end{array}$ \\
\hline aircraft & 5 & 100 & 300 & 17628.397 \\
\hline helicopter & 8 & 80 & 200 & 13935.972 \\
\hline vessel (type A) & 6 & 50 & 65 & 6678 \\
\hline vessel (type B) & 5 & 32 & 38 & 6678 \\
\hline vessel (type C) & 3 & 32 & 38 & 6678 \\
\hline vessel (type D) & 3 & 32 & 38 & 6678 \\
\hline vessel (type E) & 4 & 50 & 65 & 6678 \\
\hline vessel (type F) & 6 & 50 & 65 & 6678 \\
\hline vessel (type G) & 5 & 50 & 65 & 6678 \\
\hline vessel (type $\mathrm{H}$ ) & 3 & 50 & 65 & 6678 \\
\hline vessel (type I) & 3 & 50 & 65 & 6678 \\
\hline vessel (type J) & 4 & 50 & 65 & 6678 \\
\hline vessel (type $\mathrm{K}$ ) & 5 & 60 & 80 & 6678 \\
\hline vessel (type L) & 5 & 20 & 20 & 6678 \\
\hline UAV & 7 & 125 & 450 & 19709.619 \\
\hline
\end{tabular}


sets and their features such as: the type of asset, the number of each asset in the scenario, the arbitrary moving cost it will take to operate each asset per kilometer, the assumed fixed speeds at which they operate and the assumed radius visible to an observer operating the asset (which will be discussed in detail later).

\subsubsection{The Geohash system}

The Geohash ${ }^{2}$ system is an elegant geocoding system created by Gustavo Niemeyer in 2008 and placed in public domain. Originally created as component in a url shortening service, geohashes work by encoding a geographical location or a group of locations into a single alpha-numeric string where each additional character improves on the precision of the location as shown in figure 4.2. From figure 4.2, we can see that the
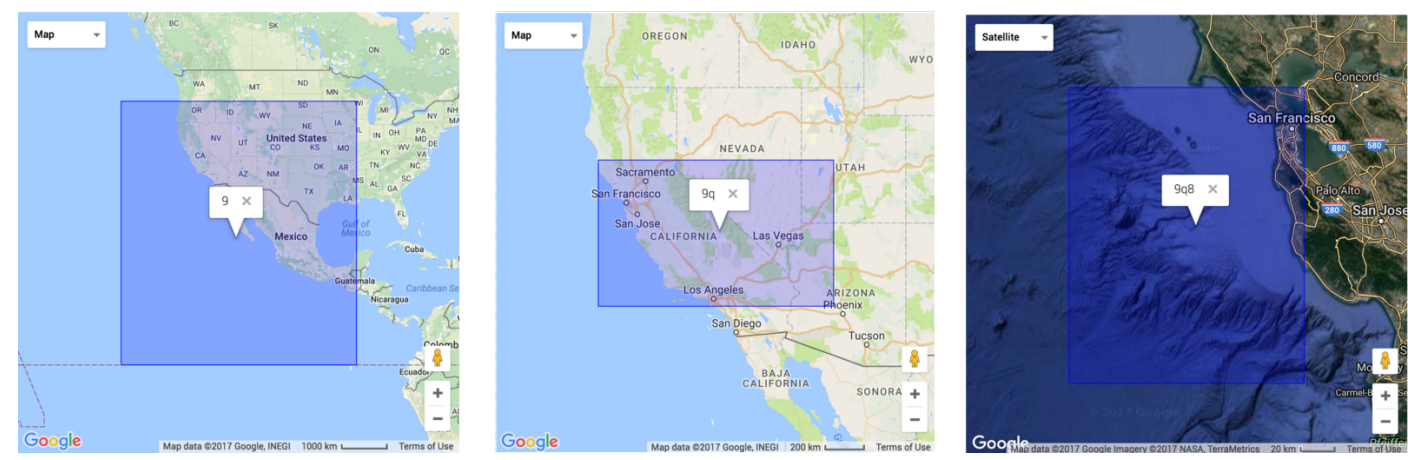

Figure 4.2: Geohash precision example

encoded location is graphically represented as a rectangular cell and all points in the cell belong to the same encoding. Also, as the precision improves, the cell becomes smaller.

Geohashes are a type of grid spatial index, where the world is recursively divided into smaller and smaller grids. For example, let's assume we want to encode the location in figure 4.3 using the geohash system. We start with the entire planet

\footnotetext{
${ }^{2}$ https://en.wikipedia.org/wiki/Geohash
} 
and the first thing we do is to divide it into two halves along the prime meridian as shown in figure 4.3 and assign a binary value of 0 to the first half containing latitudes $[-180,0]$ and assign the binary value 1 to the second half containing latitudes in the range $[0,180]$. Thus, the half containing our location becomes the first bit of our string.

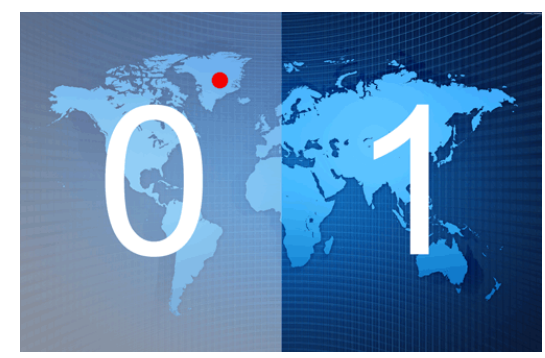

Figure 4.3: Geohash precision example

If we are satisfied at this point, we could say the geohash encoded string of our location is 0 , but this is not much use to us and it is the lowest bit resolution. We can now sub divide the "0" half again into two halves along the equator and add the second bit by assigning the binary values 0 and 1 to the two halves as shown in figure 4.4. Again, we can see that our point of interest is located in the zero half of

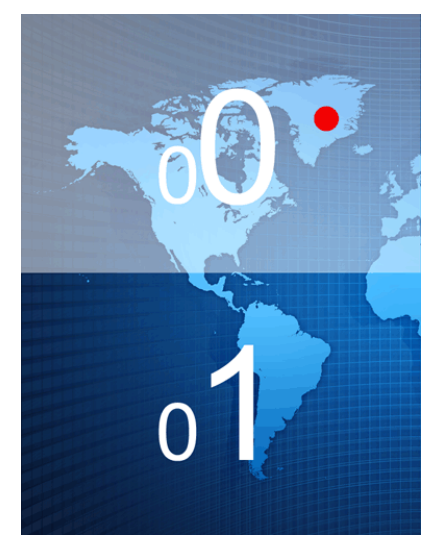

Figure 4.4: Geohash precision example

the sub division and the encoded string, " 00 " has a higher bit resolution of 2 . We 
can improve the precision again by further sub dividing the half where our location lies along the longitude and assign 0 and 1 binary values as shown in figure 4.5. We

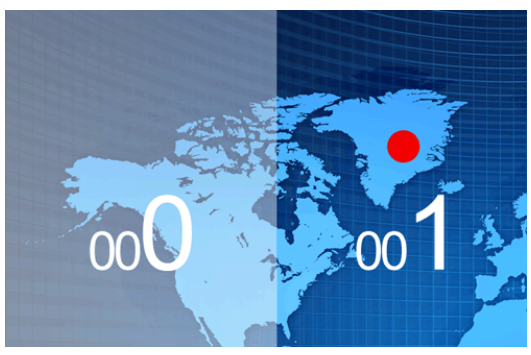

Figure 4.5: Geohash precision example

can keep repeating this sub division process, alternating between dividing along the latitude and longitude until we achieve a desired string precision. If we continue sub dividing our example to a street-view level our geohash bit will be:

0010110101011100011000110001101111000111

To encode it as alpha-numeric characters (32 bit encoding), we separate the bits into groups of 5 and convert each group to a character as shown below.

$$
\begin{array}{llllllll}
00101 & 10101 & 01110 & 00110 & 00110 & 00110 & 11110 & 00111
\end{array}
$$

$\begin{array}{llllllll}5 & \mathrm{p} & \mathrm{f} & 6 & 6 & 6 & \mathrm{y} & 7\end{array}$

The final encoded string is "5pf666y7" and it has a precision of 8 which has a rectangular cell dimension of approximately $38.2 \mathrm{~m} \times 19 \mathrm{~m}$. Table 4.2 shows the metric dimensions for the rectangular cells covered by encoded geohash strings of various lengths. 
Table 4.2: Geohash precision cell dimensions

\begin{tabular}{|c|c|}
\hline 1 & $5,009.4 \mathrm{~km} \times 4,992.6 \mathrm{~km}$ \\
\hline 2 & $1,252.3 \mathrm{~km} \times 624.1 \mathrm{~km}$ \\
\hline 3 & $156.5 \mathrm{~km} \times 156 \mathrm{~km}$ \\
\hline 4 & $39.1 \mathrm{~km} \times 19.5 \mathrm{~km}$ \\
\hline 5 & $4.9 \mathrm{~km} \times 4.9 \mathrm{~km}$ \\
\hline 6 & $1.2 \mathrm{~km} \times 609.4 \mathrm{~m}$ \\
\hline 7 & $152.9 \mathrm{~m} \times 152.4 \mathrm{~m}$ \\
\hline 8 & $38.2 \mathrm{~m} \times 19 \mathrm{~m}$ \\
\hline 9 & $4.8 \mathrm{~m} \times 4.8 \mathrm{~m}$ \\
\hline 10 & $1.2 \mathrm{~m} \times 59.5 \mathrm{~cm}$ \\
\hline 11 & $14.9 \mathrm{~cm} \times 14.9 \mathrm{~cm}$ \\
\hline 12 & $3.7 \mathrm{~cm} \times 1.9 \mathrm{~cm}$ \\
\hline
\end{tabular}

\subsubsection{The multi-objective optimization problem}

The optimization problem we want to solve in this simulation is to choose the optimum group of assets among all the 72 available ones which are capable of responding to an initial detection of an IUU fishing event with the best optimization values for each criteria (e.g. minimizing cost) and present the trade-off solutions to a decision maker. In essence, we want to model the response in such a way that the optimization algorithms will figure out the best combination of assets that can perform the task while optimizing the resources.

\subsubsection{Solution encoding}

We encode a candidate response to an IUU fishing event as a bi-layer, integer-valued solution of variable length, meaning the solution sizes range from $1-5$ assuming that no more than 5 assets will be realistically dispatched by the decision maker to respond 
to an IUU fishing event. An example of a solution chromosome is depicted in figure 4.6 where each gene (a column in the chromosome) in the chromosome represents a particular asset that could be used to respond to the incident.

\begin{tabular}{|c|c|c|c|}
\hline 15 & 9 & 34 & $\checkmark$ asset index selection layer \\
\hline 72 & 25 & 56 & travel distance (in \%) layer \\
\hline
\end{tabular}

Figure 4.6: A bi-layer encoded potential response member of the population

From figure 4.6, a single member of the population has two components, namely: the asset and asset's travel distance that will also be evolved. The top layer is encoded with integer values indicating the indices of the selected assets. These values range from 1 to the number of assets $N=72$. The bottom layer is encoded with an integer representing the percentage of the distance to the IUU fishing vessel that the particular asset will have to travel as part of the response as the assets are assumed to travel in a straight line. For instance, in the encoding portrayed in figure 4.6, asset 15 will have to travel $72 \%$ of the distance towards the target (IUU fishing vessel), asset 9 will travel $25 \%$ of its distance to the target and asset 34 will relocate itself closer to the target after traveling $56 \%$ of its initial distance to the target.

\subsubsection{Decision objectives}

For our simulation, we have three trade-off decision objectives (or criteria) that we want to optimize. The first one is the Cost objective. We define the cost objective at the total cost of all assets in a solution in responding to the IUU event defined as shown in equation 4.2 and our goal is to minimize the objective.

$$
\text { Minimize Cost }=\sum_{a \in A} \operatorname{CPK}\left(a_{i}\right) \times \operatorname{DTT}\left(a_{i}\right)
$$


where CPK is the cost per kilometer covered by the asset $a$; DTT is the current distance to target (i.e. distance to the IUU fishing vessel) for asset $a_{i}$.

The second objective is the Proximity to Target (PTT) objective. We define the PTT objective as the sum of the combined distances of each asset in the solution to the IUU fishing vessel. Our aim with the objective is to find the best solution that minimizes it. The objective is defined in equation 4.3.

$$
\text { Minimize PTT }=\sum_{a \in A} \operatorname{DTT}\left(a_{i}\right)
$$

where DTT is likewise the current distance to target for asset $a_{i}$. At a first glance, it might seem like the cost and proximity to target objectives are related and hence cancel each other out, but this is not always the case. For example, moving any of the aerial vehicles in table 4.1 over a short distance will incur a high cost and still have a large proximity to target while 3 small boats of type B can cover larger distances with a lower cost and still have a lower proximity.

The third objective is the Probability of confirmed Detection (PDetection) objective which is defined as the probability of confirming the detection of the offending fishing vessel inside the unauthorized fishing zone which is important to the law enforcement authorities for prosecution purposes. To evaluate the PDetection objective, the first thing we do is encode both the unauthorized fishing zone and the visibility radius around the assets in the solution as shown in figure 4.7.

For our simulation, the unauthorized fishing zone and the assets' visibility radius were encoded to a string precision of 6 characters which is about $1.2 \mathrm{~km} \times 609.4 \mathrm{~m}$ as shown in table 4.2. The next thing to do in evaluating the PDetection objective is to count the total number of each asset's visibility geohashes that overlap those of the unauthorized fishing zone and divide the sum by the total number of geohashes in 


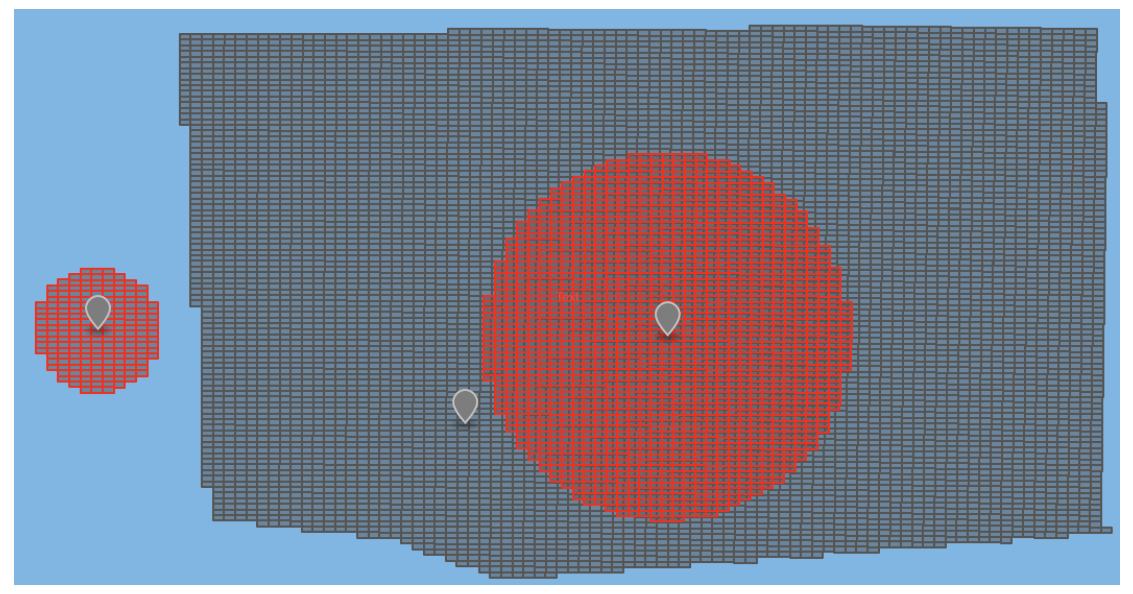

Figure 4.7: Geohash encoding of the fishing zone and the assets

the unauthorized lobster fishing area (LFA) as shown in equation 4.4.

$$
\text { Maximize PDetection }=\frac{\sum_{a \in A} \text { Overlapping Geohashes }\left(a_{i}\right)}{\text { Total number of Geohashes in LFA }}
$$

\subsubsection{Algorithm parameters}

For the NSGA-II algorithm, we initialized the size of the population $N$ to 100 . The selection operation of parents for recombination or crossover is done using binary tournament selection with replacement. Recombination of the selected parents is done using a crossover operation where half of the non-matching members are swapped between the 2 member solutions and the probability that the crossover operator is applied to the chosen parent members is 0.9. To mutate the offspring produced from the recombination operation, we replace one of the members in the solution with a randomly chosen asset member and percentage distance travelled.

The size of the population for the NSGA-III algorithm was also initialized to 100 solutions because we were sponsored by a company called Larus technologies here in Ottawa and they have a product that uses a population size of 100 . For our simulation, $\mathrm{H}$ is set to 91 reference points (computed using equation 4.5 as described 
in chapter 3) so we can have the number of reference points close to the population size.

$$
\mathrm{H}=\left(\begin{array}{c}
\mathrm{M}+\text { divisions }-1 \\
\text { divisions }
\end{array}\right)
$$

where $\mathrm{M}$ is the number of objectives (3 in our problem) and the number of divisions is arbitrarily set to 12 . The selection, recombination and mutation operators are the same as those for the NSGA-II algorithm.

The population size and genetic operators (selection, recombination and mutation operators) of the PESA-II algorithm as the same as those described above. The size of the archive set is set to 100 and the number of bisections in the adaptive grid archive is arbitrarily set to 8 . Finally, for the SPEA2 algorithm, the number of offsprings generated at every iteration is set to 100 and the crowding operator based on the kth-nearest operator is set to 1 . The four algorithms were configured to run for a maximum of 100, 000 Number of Function Evaluations (NFEs).

\subsubsection{Simulation results}

\subsubsection{The NSGA-II algorithm}

Figure 4.8 shows the pareto front generated from a single simulation run of the NSGAII algorithm applied to the IUU fishing multi-objective problem described in section 4.2.1. The authors of the NSGA-II algorithm in [28] claim that the algorithm emphasizes the non-dominated pareto optimal solutions. We can see from the figure in 4.8 that the solutions on the pareto front consist of a set of non-dominated solutions with trade-offs in the three objectives, namely: the moving cost, proximity to target and the probability of detection. Figure 4.9 shows a graphical illustration of one of the solutions in the final pareto front of figure 4.8 which shows a solution with a very 


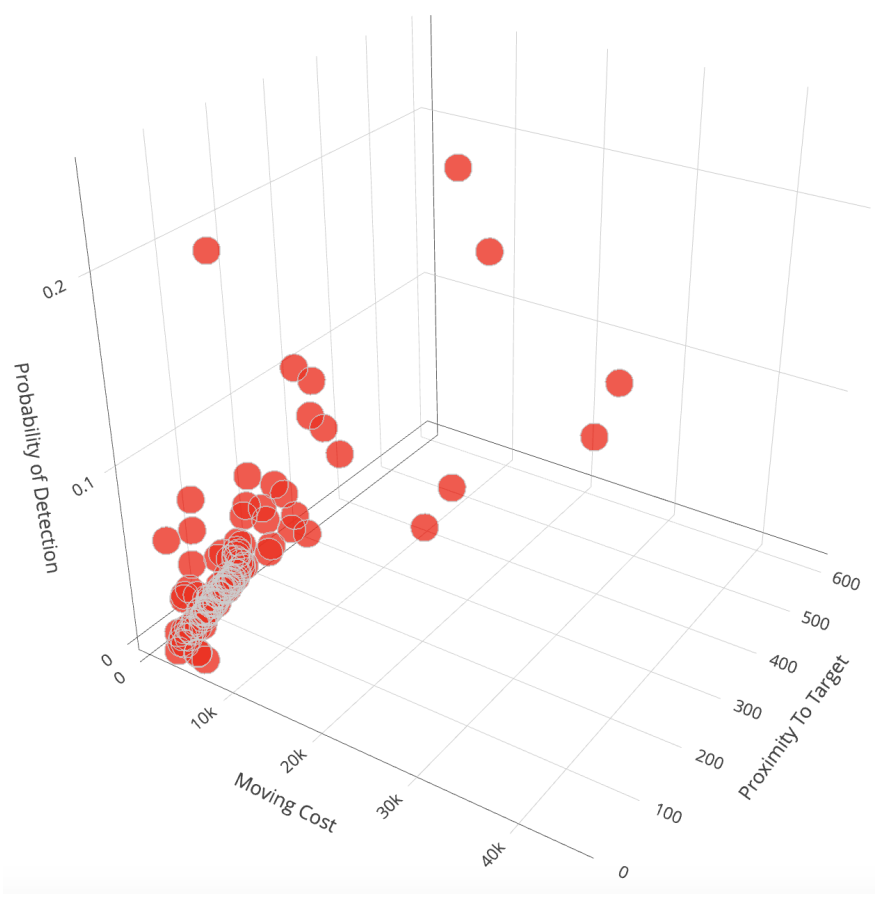

Figure 4.8: Pareto front generated using the NSGA-II algorithm

high Cost of $\$ 33,661.52$, PTT of $69.769 \mathrm{~km}$ and PDetection of 0.245 .

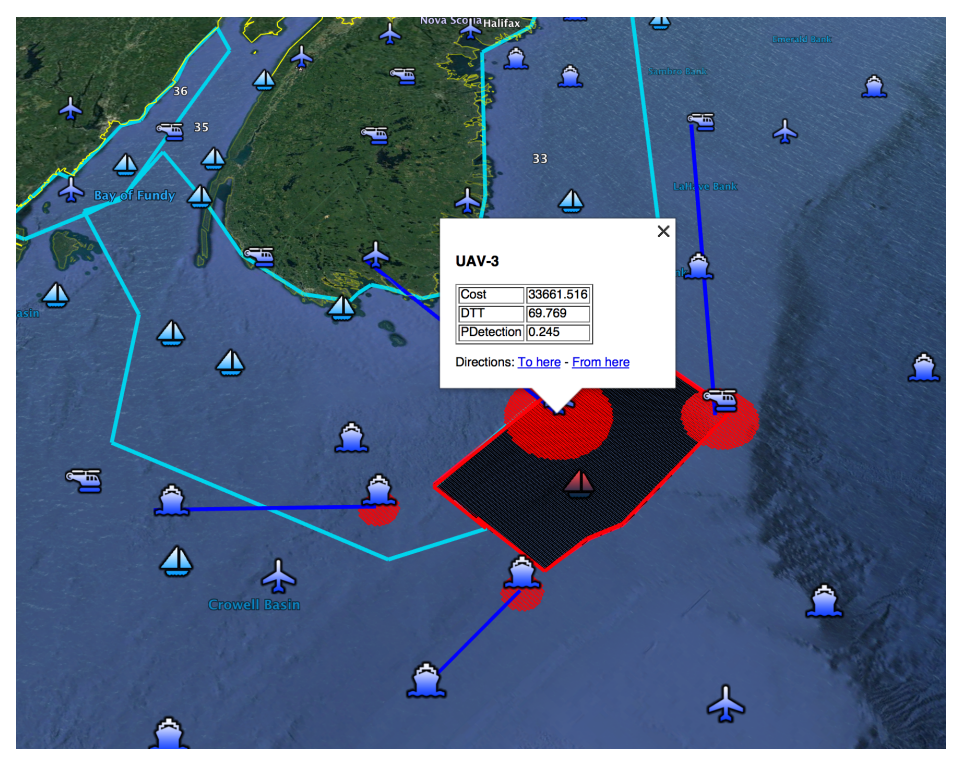

Figure 4.9: Graphical illustration of a solution in the NSGA-II pareto front 


\subsubsection{The NSGA-III algorithm}

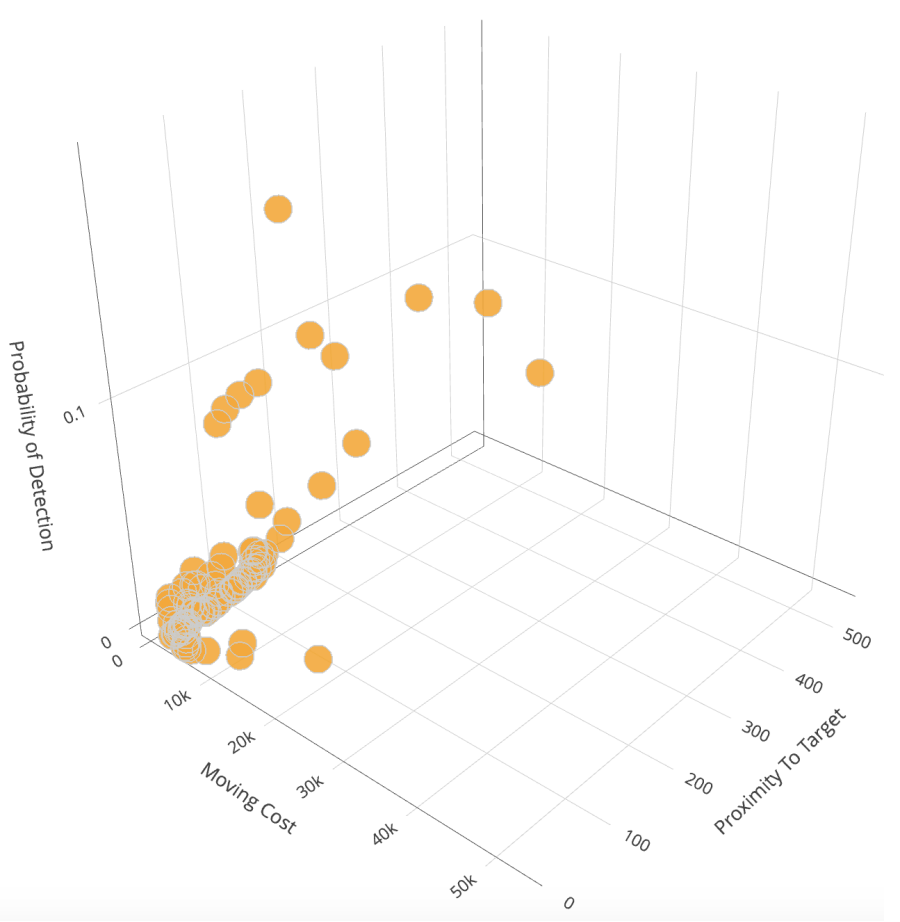

Figure 4.10: Pareto front generated using the NSGA-III algorithm

Figure 4.10 shows the pareto front generated from a single simulation run of the NSGA-III algorithm applied to the IUU fishing multi-objective problem described in section 4.2.1. The authors of the NSGA-III algorithm in [29] claim that the algorithm is an improvement over the NSGA-II algorithm with the new reference point approach. This improvement is not really evident in the plot of figure 4.10, but like the NSGA-II algorithm the solutions consist of a set of non-dominated solutions with trade-offs in the three objectives. Figure 4.11 shows a graphical illustration of a solution with a Cost of $\$ 11,831.385$, PTT of $28.296 \mathrm{~km}$ and PDetection of 0.112 . 


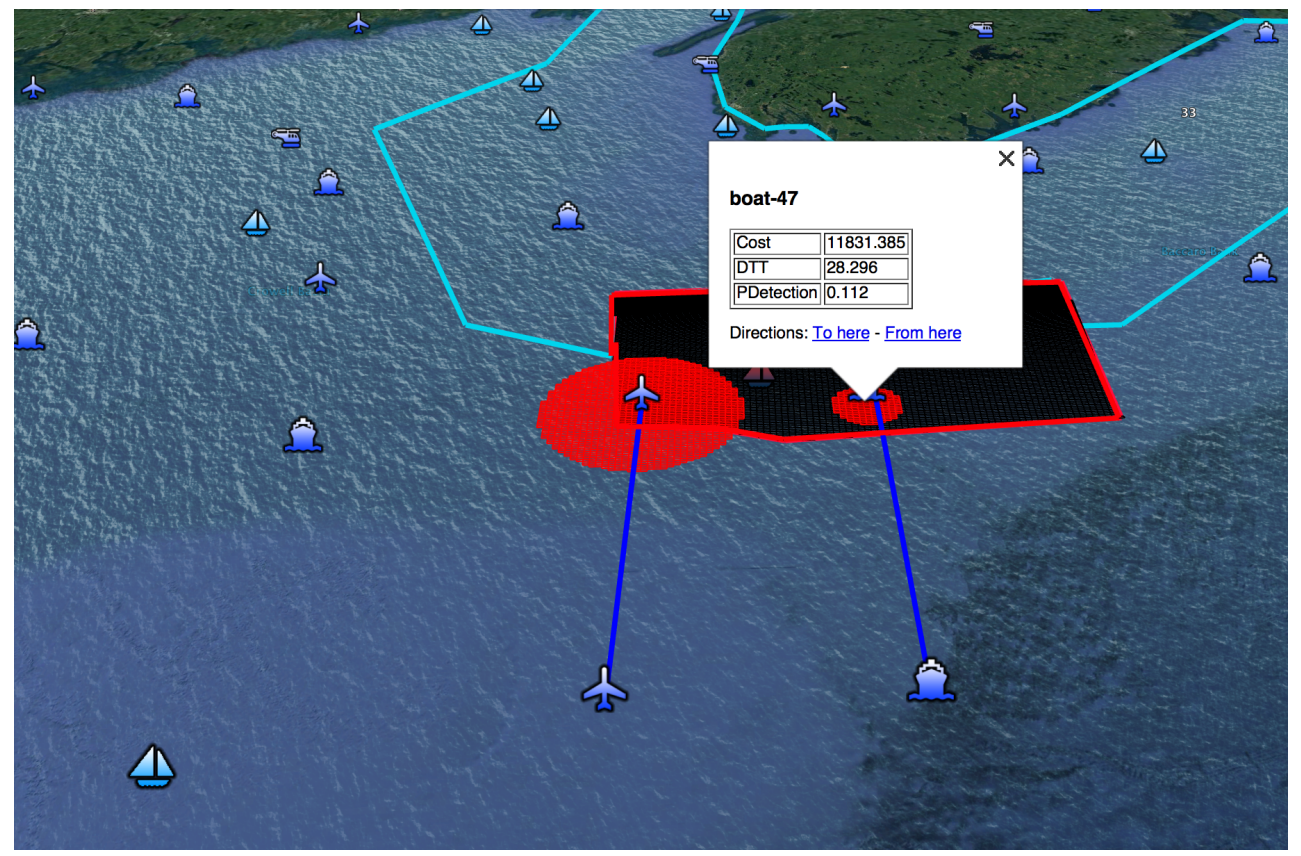

Figure 4.11: Graphical illustration of a solution in the NSGA-III pareto front

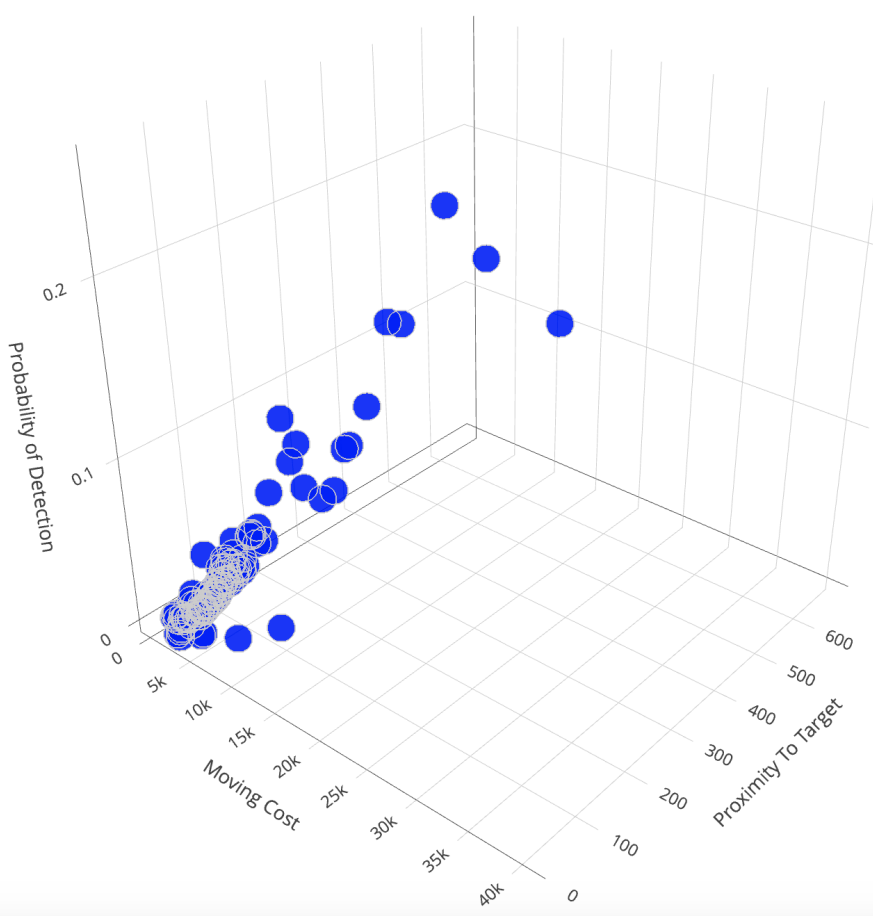

Figure 4.12: Pareto front generated using the SPEA2 algorithm 


\subsubsection{The SPEA2 algorithm}

Figure 4.12 shows the pareto front generated from a single simulation run of the SPEA2 algorithm applied to the IUU fishing multi-objective problem described in section 4.2.1. The authors of the SPEA2 algorithm in [31] claim that the algorithm will emphasize the pareto-optimal solutions at each generation using the archive set. By observing the pareto front in figure 4.12, we can see that the algorithm does a good job at optimizing the objectives, but until we statistically compare the algorithms, we cannot say if it has better pareto optimal solutions when compared to the other algorithms. Figure 4.13 shows a graphical illustration of a solution with a Cost of $\$ 3,406.66$, PTT of $4.466 \mathrm{~km}$ and PDetection of 0.066 .

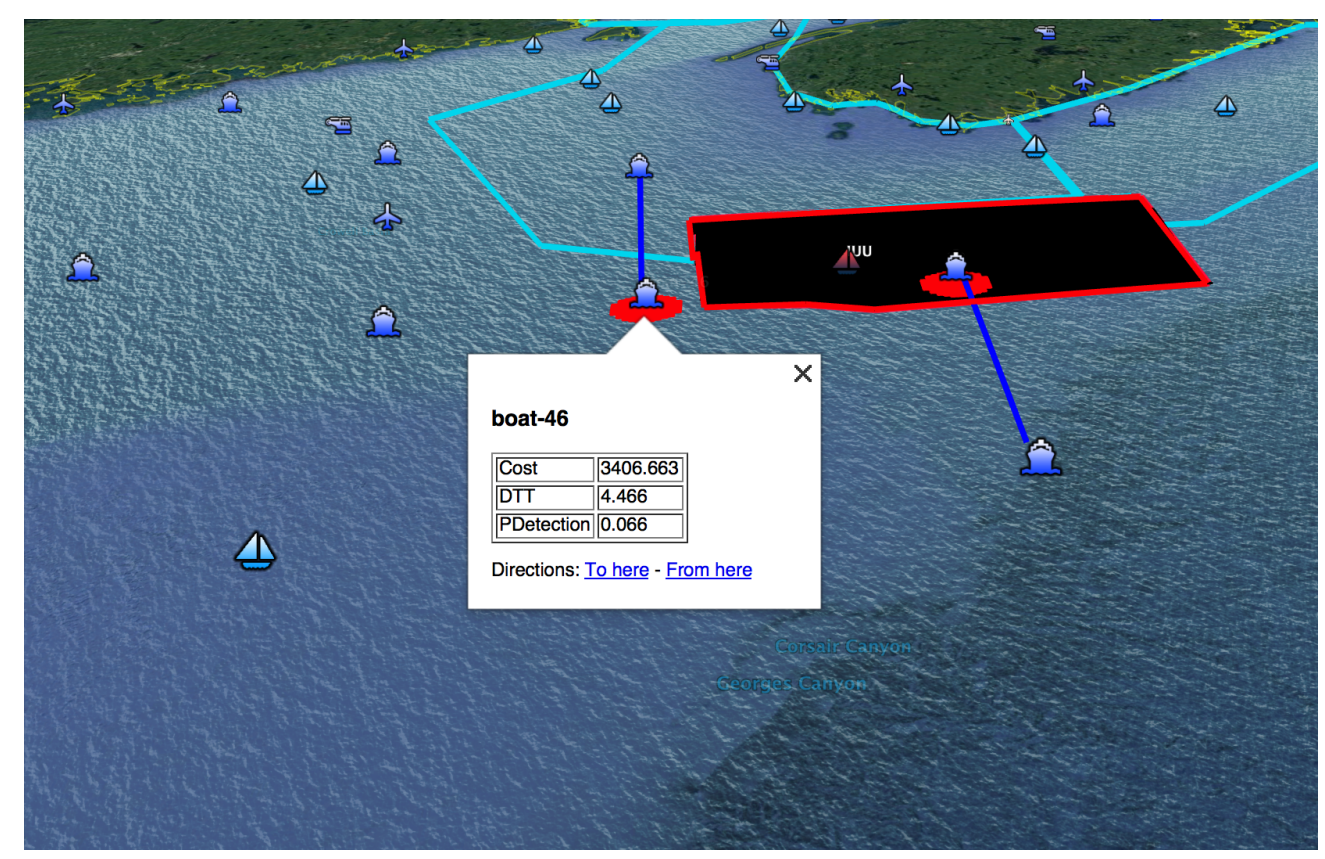

Figure 4.13: Graphical illustration of a solution in the SPEA2 pareto front 


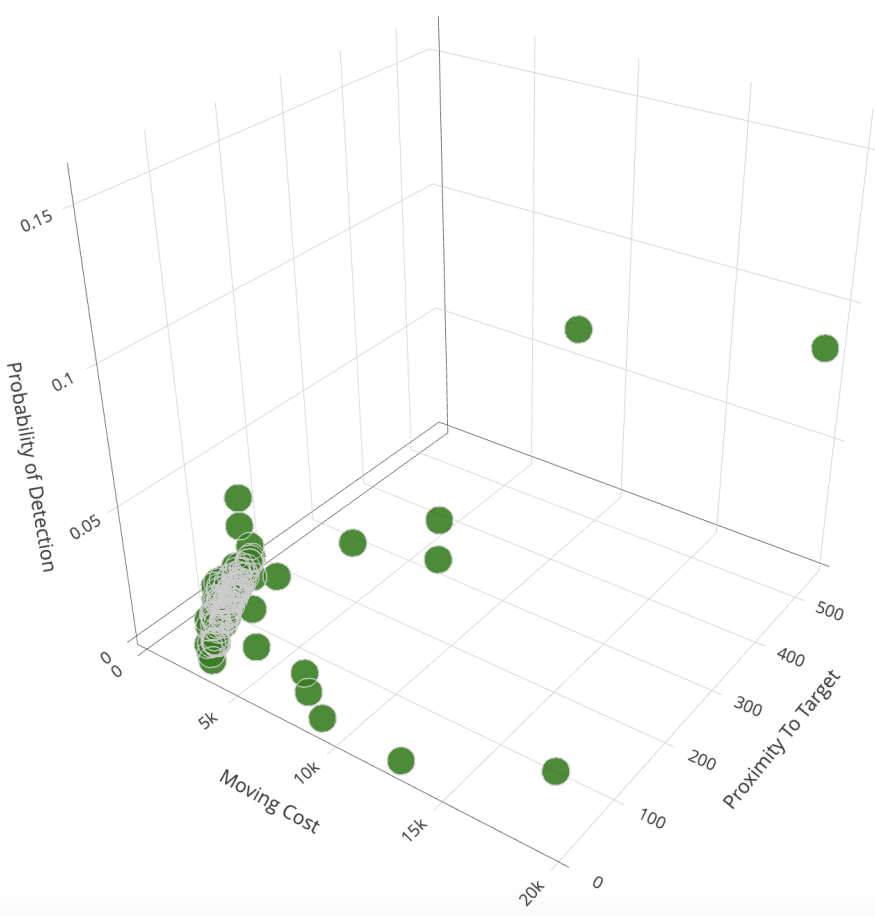

Figure 4.14: Pareto front generated using the PESA-II algorithm

\subsubsection{The PESA-II algorithm}

Figure 4.14 shows the pareto front generated from a single simulation run of the PESA-II algorithm applied to the IUU fishing multi-objective problem described in section 4.2.1. The authors of the algorithm claimed that the new selection technique introduced in [33] together with the archive set that stores the pareto-optimal solutions at each generation will produce very good pareto optimal solutions. Observing the pareto front in figure 4.14, we can see that the PESA-II algorithm does comparatively well in optimizing the objectives. Figure 4.13 shows a graphical illustration of a solution with a Cost of $\$ 44,057.19$, PTT of $35.64 \mathrm{~km}$ and PDetection of 0.19. 


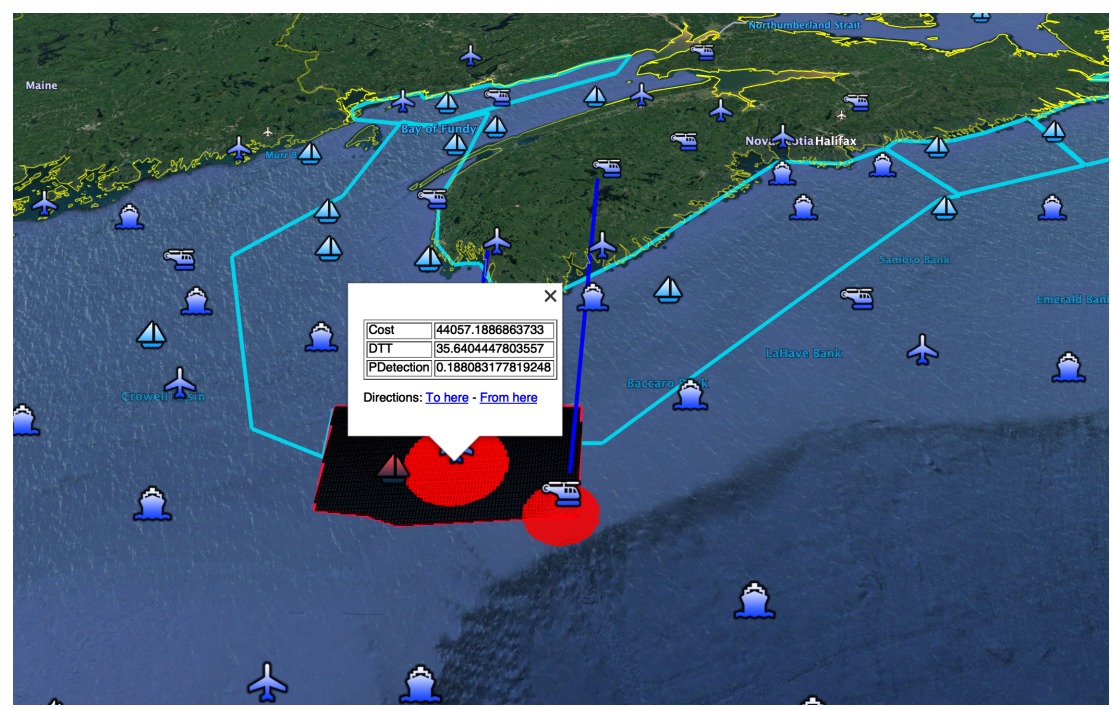

Figure 4.15: Graphical illustration of a solution in the PESA-II pareto front

\subsubsection{Comparing the algorithms}

\subsubsection{Performance of the algorithms using the Hypervolume metric}

Comparing their performances produced the following results shown in equations 4.6 - 4.9. Figure 4.16 shows the graphical illustration of the hypervolume performance metric comparison. From the figure, we can see that the algorithm perform similarly well with the NSGA-II algorithm slightly edging out the other three algorithms. From equation 4.6, we can see that the NSGA-II, PESA-II and SPEA2 algorithms are statistically indifferent with $95 \%$ confidence. Also from equation 4.7 , we can see that the NSGA-III and PESA-II algorithm are statistically indifferent, also with a $95 \%$ confidence.

Hence, we can conclude that from our simulations all the four algorithms perform similarly well and that none of them is statistically superior over the others. Looking into the computational complexities of the algorithms, the NSGA-II algorithm has the lowest computational complexity of $\left(O\left(M N^{2}\right)\right.$ while still performing relatively well when compared to the others. This then begs the question, "Are the little 
improvements that other algorithms claim to have over the NSGA-II algorithm worth the computational complexity sacrifice in complex applications?".

NSGA-II :

Hypervolume:

Min: $\quad 0.06601$

Median: 0.20256

Max: $\quad 0.37137$

Count: 50

Indifferent: [PESA-II, SPEA2]

NSGA-III :

Hypervolume:

Min: $\quad 0.03059$

Median: 0.16238

Max: 0.35883

Count: 50

Indifferent: [PESA-II] 
PESA-II :

Hypervolume :

Min: $\quad 0.05684$

Median: 0.17639

Max : 0.50953

Count: 50

Indifferent: [NSGA-II, NSGA-III]

SPEA2 :

Hypervolume:

Min: $\quad 0.07143$

Median: 0.23166

Max: $\quad 0.30960$

Count: 50

Indifferent: [NSGA-II]

\subsubsection{Effects of the diversity operations of the algorithms}

Similar to what we did in chapter 3, we ran each algorithm 100 times (where each run is for 100,000 function evaluations) for a population size of 100 each and computed the average number of times the diversity operations of each algorithm influenced the new generation's population. From table 4.3, we can see how many times on average (to the nearest whole number) the diversity operation of each algorithm was executed. For example, the crowding-distance diversity operation of the NSGA-II algorithm came into play an average of 25,634 times out of 10,000,000 generation iterations, 


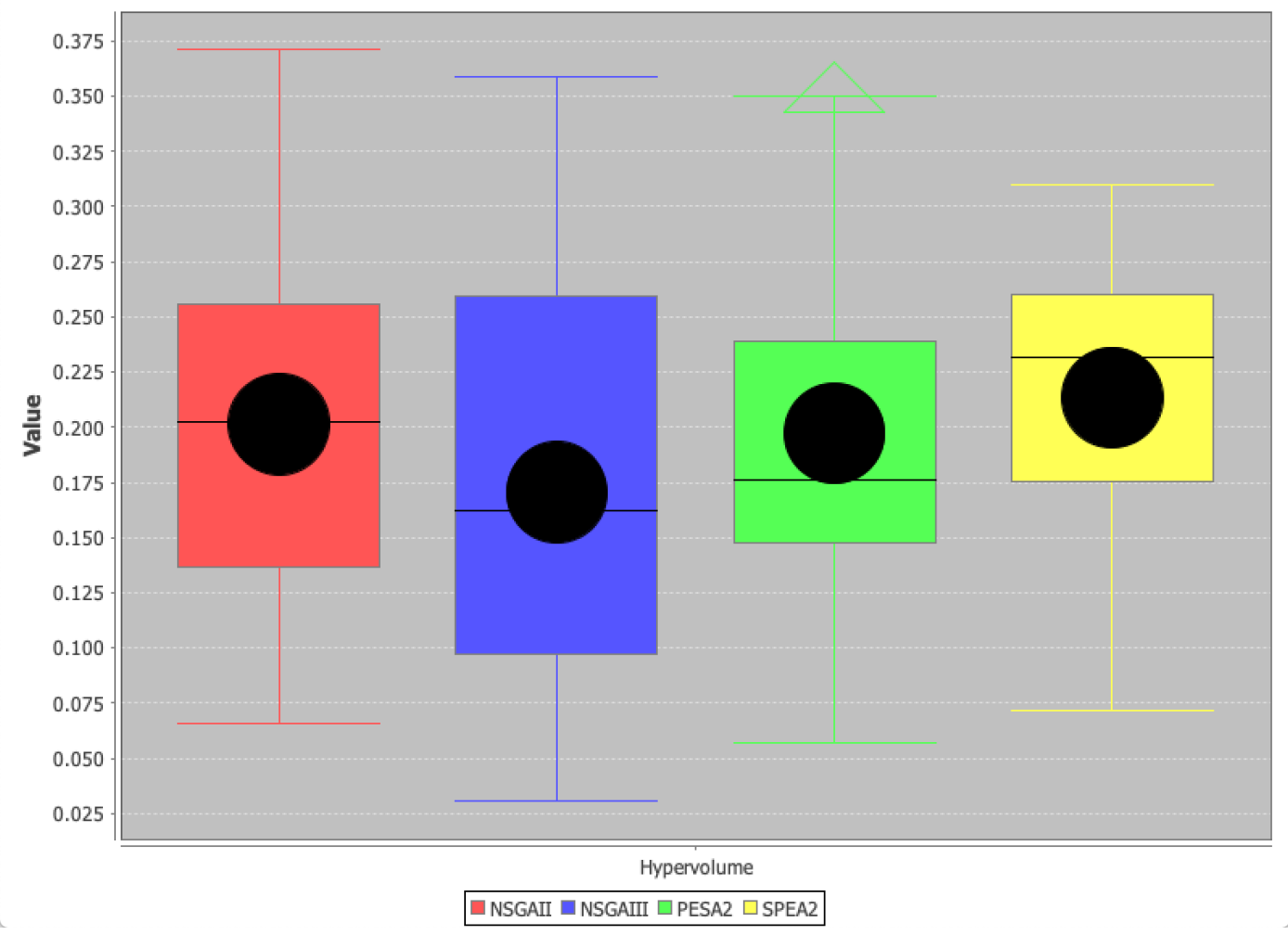

Figure 4.16: Graphical plot of the hypervolume metric for the IUU scenario 
while the reference point-based diversity operation of the NSGA-III algorithm came into effect 32,452 times out of $10,000,000$ iterations.

Table 4.3: Average diversity operation count

\begin{tabular}{|c|c|c|c|c|}
\hline Algorithm & NSGA-II & NSGA-III & SPEA2 & PESA-II \\
\hline Average count & 25,634 & 32,452 & 29,786 & 30,134 \\
\hline
\end{tabular}

Once again, we carried out another simulation of the algorithms using the same initial parent population for each and forcing the same selection, crossover and mutation operations at every generation. Again, we did this to study the effect of the diversity preservation operation of each algorithm on a small population size. From the test, we observed that the four algorithms produce the same solutions as shown in equations 4.10 - 4.14. The tables below each equation show the assets and their corresponding percentage distance travelled.

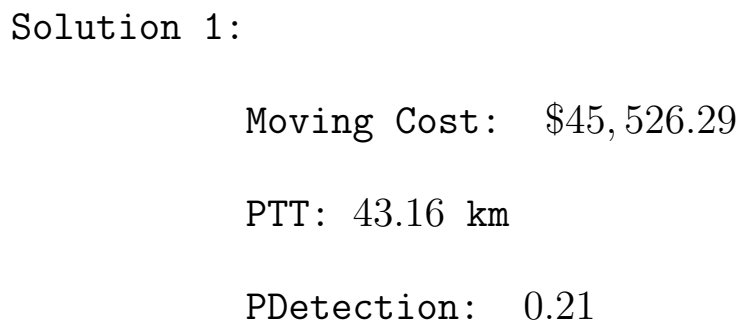

\begin{tabular}{|c|c|c|c|c|}
\hline Assets & UAV & helicopter & vessel (type K) & vessel (type D) \\
\hline Distance travelled (\%) & $90 \%$ & $96 \%$ & $84 \%$ & $97 \%$ \\
\hline
\end{tabular}


Solution 2:

Moving Cost: $\quad \$ 3,997.36$

PTT: $598.61 \mathrm{~km}$

PDetection: 0.17

\begin{tabular}{|c|c|c|}
\hline Assets & vessel (type C) & vessel (type J) \\
\hline Distance travelled (\%) & $10 \%$ & $46 \%$ \\
\hline
\end{tabular}

Solution 3:

Moving Cost: $\quad \$ 6,866.51$

PTT: $1.72 \mathrm{~km}$

PDetection: 0.004

\begin{tabular}{|c|c|c|}
\hline Assets & vessel (type K) & vessel (type L) \\
\hline Distance travelled (\%) & $99 \%$ & $99 \%$ \\
\hline
\end{tabular}

Solution 4:

Moving Cost: $\$ 381.58$

PTT: $376.88 \mathrm{~km}$

PDetection: 0.03

\begin{tabular}{|c|c|c|}
\hline Assets & aircraft & vessel (type J) \\
\hline Distance travelled (\%) & $1 \%$ & $2 \%$ \\
\hline
\end{tabular}




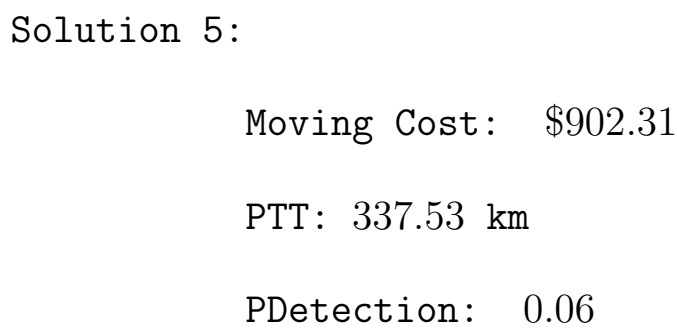

Figure 4.17 shows the pareto front showing the approximate solutions. In the same

\begin{tabular}{|c|c|c|}
\hline Assets & vessel (type B) & vessel (type B) \\
\hline Distance travelled (\%) & $1 \%$ & $12 \%$ \\
\hline
\end{tabular}

way, we observe that the diversity operations of the algorithms do not come into play when the population size is small. In conclusion, if one has a relatively large population then the solutions naturally fill out the pareto front, even if there is crowding, but if there is a small population then crowding is unlikely anyway.

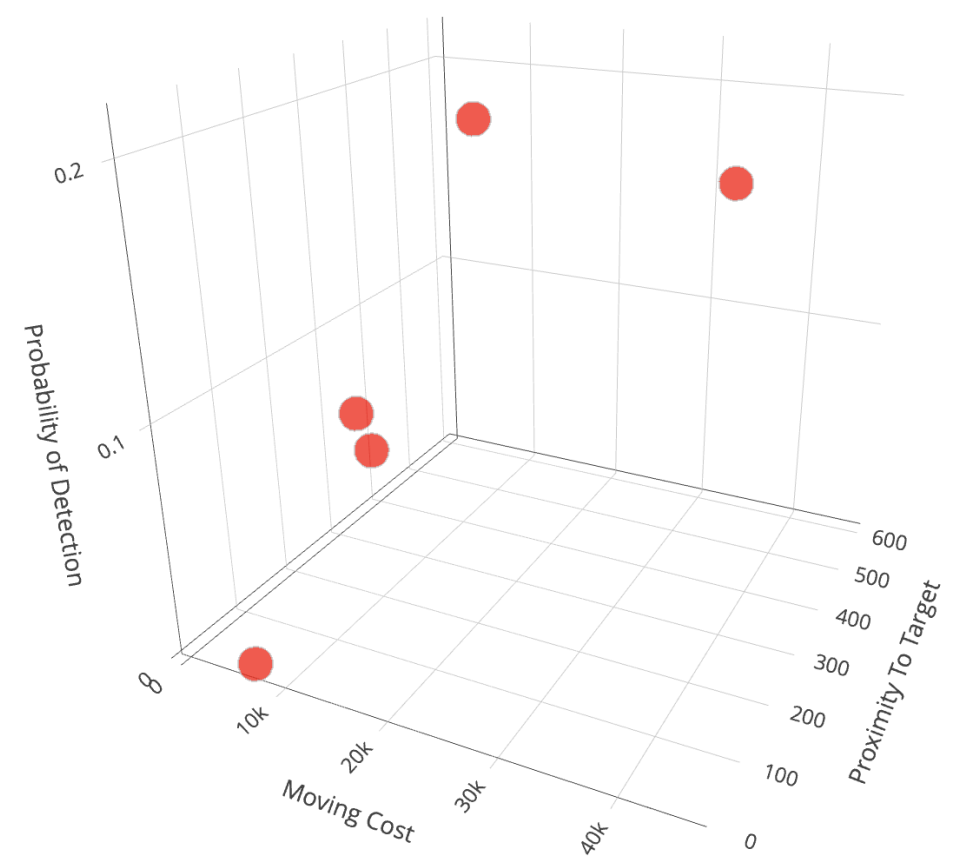

Figure 4.17: Pareto front produced using a small population size 


\section{Chapter 5}

\section{Conclusion and Future work}

\subsection{Conclusion}

We examine four of the most frequently cited Multi-Objective Evolutionary Algorithms (MOEAs), namely: the NSGA-II, NSGA-III, SPEA2 and PESA-II algorithms. We investigate the inherent differences between the algorithms first with regard to a relatively simple example in chapter 3 and then a more complex simulation by employing the algorithms in automatically generate potential responses to IUU fishing incidents.

In chapter 3, we examined thoroughly, the differences between these algorithms concluding that the major differences occur in the way they each approach diversity in the population. In chapter 4, we proposed a methodology to responding to IUU fishing incidents using a simulated IUU fishing scenario along the Canadian Atlantic coast, where a few of the responses obtained were discussed. The exploration of the search space of possible responses was aimed at discovering the trade-off among three decision objectives, namely (1) the proximity to the target (IUU fishing vessel),

(2) the total cost of the response for all engaged assets and (3) the probability of confirming the detection of the offending vessel inside the unauthorized fishing zone, 
which is important for prosecution purposes.

We conclude from examining and analyzing the four algorithms using the simple and complex maritime domain problems that the diversity operations of these algorithms are of virtually no benefit in these applications. Furthermore, the literature does not indicate that diversity is an issue for any practical problems; as such if one has a relatively large population then the solutions naturally fill out the pareto front, even if there is crowding, but if there is a small population then crowding is unlikely anyway. Additionally, the Pareto Archive Set discovered by four very popular EMOO algorithms confirms that these techniques are indeed suitable to identify a promising set of candidate responses in a reasonable amount of time. To the best of our knowledge, this is the first time EMOO techniques have been applied to respond to IUU fishing incidents.

\subsection{Future work}

For the multi-objective evolutionary approach, future work directions involve: the design of more complicated types of coordinated responses by taking into account predictable movement patterns that both the IUU fishing vessel and the responding assets could execute, a more comprehensive evaluation based on more synthetic and possibly real-world scenarios and comparing the automatic responses generated by the EMOO approach with those generated by human experts and endow the proposed method of an adaptive learning mechanism that takes the operator's feedback into consideration. 


\section{List of References}

[1] Bureau of Political-Military Affairs, "The national strategy for maritime security." https://www.state.gov/t/pm/rls/othr/misc/255321.htm, September 2005. [Online; accessed 25-September-2018].

[2] R. Abielmona, "Tackling Big Data in maritime domain awareness," Vanguard, pp. 42-43, August-September 2013.

[3] ISO Central Secretary, "Quality management and quality assurance - Vocabulary," Standard ISO 8402:1995/BS 4778, International Organization for Standardization, Geneva, CH, 1995.

[4] International Association of Classification Societies, "A guide to risk assessment in ship operations." http://www.iacs.org.uk/download/1927. [Online; accessed 1-June-2019].

[5] Fisheries and O. Canada, "Illegal, Unreported and Unregulated (IUU) fishing." http://www.dfo-mpo.gc.ca/international/isu-iuu-eng.htm. [Online; accessed 27-February-2018].

[6] FAO, "The state of world fisheries and aquaculture: contributing to food security and nutrition for all." http://www.fao.org/3/a-i5555e.pdf, 2016. [Online; accessed 10-July-2017].

[7] T. Akinbulire, H. Schwartz, R. Falcon, and R. Abielmona, "A reinforcement learning approach to tackle illegal, unreported and unregulated fishing," in 2017 IEEE Symposium Series on Computational Intelligence (SSCI), pp. 1-8, November 2017 .

[8] W. O. Review, "Illegal fishing." http://worldoceanreview.com/en/wor-2/ fisheries/illegal-fishing/. [Online; accessed 10-July-2017]. 
[9] R. Falcon, A. Nayak, and R. Abielmona, "An evolving risk management framework for wireless sensor networks," in Computational Intelligence for Measurement Systems and Applications (CIMSA), 2011 IEEE International Conference on, pp. 1-6, IEEE, 2011.

[10] R. Falcon and R. Abielmona, "A response-aware risk management framework for search-and-rescue operations," in Evolutionary Computation (CEC), 2012 IEEE Congress on, pp. 1-8, IEEE, 2012.

[11] J. McCausland, R. Abielmona, R. Falcon, A.-M. Cretu, and E. Petriu, "Auctionbased node selection of optimal and concurrent responses for a risk-aware robotic sensor network," in Robotic and Sensors Environments (ROSE), 2013 IEEE International Symposium on, pp. 136-141, IEEE, 2013.

[12] R. Falcon, R. Abielmona, S. Billings, A. Plachkov, and H. Abbass, "Risk management with hard-soft data fusion in maritime domain awareness," in Computational Intelligence for Security and Defense Applications (CISDA), 2014 Seventh IEEE Symposium on, pp. 1-8, IEEE, 2014.

[13] A. H. Razavi, D. Inkpen, R. Falcon, and R. Abielmona, "Textual risk mining for maritime situational awareness," in 2014 IEEE International Inter-Disciplinary Conference on Cognitive methods in situation awareness and decision support (cogSIMA), pp. 167-173, 2014.

[14] R. Falcon, R. Abielmona, and S. Billings, "Risk-driven intent assessment and response generation in maritime surveillance operations," in Cognitive Methods in Situation Awareness and Decision Support (CogSIMA), 2015 IEEE International Inter-Disciplinary Conference on, pp. 151-157, IEEE, 2015.

[15] A. Plachkov, R. Abielmona, M. Harb, R. Falcon, D. Inkpen, V. Groza, and E. Petriu, "Automatic course of action generation using soft data for maritime domain awareness," in Proceedings of the 2016 on Genetic and Evolutionary Computation Conference Companion, pp. 1071-1078, ACM, 2016.

[16] R. Falcon, B. Desjardins, R. Abielmona, and E. Petriu, "Context-driven dynamic risk management for maritime domain awareness," in Computational Intelligence (SSCI), 2016 IEEE Symposium Series on, pp. 1-8, IEEE, 2016.

[17] S. Mardle, S. Pascoe, and M. Tamiz, "An investigation of genetic algorithms for the optimization of multi-objective fisheries bioeconomic models," International Transactions in Operational Research, vol. 7, no. 1, pp. 33-49, 2000. 
[18] K. Deb and R. Datta, "Hybrid evolutionary multi-objective optimization and analysis of machining operations," Engineering Optimization, vol. 44, no. 6, pp. 685-706, 2012.

[19] B. D. Bunday, Basic optimisation methods. Edward Arnold London, 1984.

[20] D. Kalyanmoy et al., Multi objective optimization using evolutionary algorithms. John Wiley and Sons, 2001.

[21] J. D. Schaffer, "Multiple objective optimization with vector evaluated genetic algorithms," in Proceedings of the 1st International Conference on Genetic Algorithms, (Hillsdale, NJ, USA), pp. 93-100, L. Erlbaum Associates Inc., 1985.

[22] P. Hajela and C.-Y. Lin, "Genetic search strategies in multicriterion optimal design," Structural optimization, vol. 4, no. 2, pp. 99-107, 1992.

[23] M. P. Fourman, "Compaction of symbolic layout using genetic algorithms," in Genetic Algorithms and Their Applications: Proc. 1st Int. Conf. Genetic Algorithms, Princeton, Lawrence Erlbaum, NJ, 1985, 1985.

[24] J. Lis and Á. E. Eiben, "A multi-sexual genetic algorithm for multiobjective optimization," in Proceedings of 1997 IEEE International Conference on Evolutionary Computation (ICEC'97), pp. 59-64, IEEE, 1997.

[25] C. M. Fonseca, P. J. Fleming, et al., "Genetic algorithms for multiobjective optimization: Formulation, discussion and generalization.," in Icga, vol. 93, pp. 416423, Citeseer, 1993.

[26] D. E. Golberg, "Genetic algorithms in search, optimization, and machine learning," Addion wesley, vol. 1989, no. 102, p. 36, 1989.

[27] N. Srinivas and K. Deb, "Muiltiobjective optimization using nondominated sorting in genetic algorithms," Evolutionary computation, vol. 2, no. 3, pp. 221-248, 1994.

[28] K. Deb, A. Pratap, S. Agarwal, and T. Meyarivan, "A fast and elitist multiobjective genetic algorithm: Nsga-ii," IEEE transactions on evolutionary computation, vol. 6, no. 2, pp. 182-197, 2002.

[29] K. Deb and H. Jain, "An evolutionary many-objective optimization algorithm using reference-point-based nondominated sorting approach, part i: Solving problems with box constraints.," IEEE Trans. Evolutionary Computation, vol. 18, no. 4, pp. 577-601, 2014. 
[30] E. Zitzler and L. Thiele, "An evolutionary algorithm for multiobjective optimization: The strength pareto approach," TIK-report, vol. 43, 1998.

[31] E. Zitzler, M. Laumanns, and L. Thiele, "Spea2: Improving the strength pareto evolutionary algorithm," TIK-report, vol. 103, 2001.

[32] D. W. Corne, J. D. Knowles, and M. J. Oates, "The pareto envelope-based selection algorithm for multiobjective optimization," in International conference on parallel problem solving from nature, pp. 839-848, Springer, 2000.

[33] D. W. Corne, N. R. Jerram, J. D. Knowles, and M. J. Oates, "Pesa-ii: Regionbased selection in evolutionary multiobjective optimization," in Proceedings of the 3rd Annual Conference on Genetic and Evolutionary Computation, pp. 283290, Morgan Kaufmann Publishers Inc., 2001.

[34] T. Akinbulire, R. Falcon, R. Abielmona, and H. Schwartz, "Responding to illegal, unreported and unregulated fishing with evolutionary multi-objective optimization," in 2018 IEEE International Conference on Computational Intelligence and Virtual Environments for Measurement Systems and Applications (CIVEMSA), pp. 1-6, IEEE, 2018.

[35] J. H. Holland, "Adaptation in natural and artificial systems: an introductory analysis with applications to biology, control, and artificial intelligence," Control and artificial intelligence, 1975.

[36] I. Das and J. E. Dennis, "Normal-boundary intersection: A new method for generating the pareto surface in nonlinear multicriteria optimization problems," SIAM Journal on Optimization, vol. 8, no. 3, pp. 631-657, 1998.

[37] M. Li, J. Zheng, R. Shen, K. Li, and Q. Yuan, "A grid-based fitness strategy for evolutionary many-objective optimization," in Proceedings of the 12th annual conference on Genetic and evolutionary computation, pp. 463-470, ACM, 2010.

[38] J. Knowles and D. Corne, "Properties of an adaptive archiving algorithm for storing nondominated vectors," IEEE Transactions on Evolutionary Computation, vol. 7 , no. 2, pp. 100-116, 2003.

[39] J. Knowles and D. Corne, "On metrics for comparing nondominated sets," in Proceedings of the 2002 Congress on Evolutionary Computation. CEC'02 (Cat. No. 02TH8600), vol. 1, pp. 711-716, IEEE, 2002. 
[40] Fisheries and O. Canada, "Lobster fishing area." http://www.dfo-mpo.gc.ca/ fm-gp/sustainable-durable/fisheries-peches/lobster-homard-eng.htm. [Online; accessed 28-February-2018]. 